\title{
Shoulder girdle and forelimb myology of extant Monotremata
}

\author{
Peter P. Gambaryan, \\ Alexander N. Kuznetsov, Aleksandra A. Panyutina \& Sergey V. Gerasimov
}

\begin{abstract}
For the first time, the comparative myological analysis of the forelimbs of all living genera of Monotremata is performed. Based on thorough dissection, the degree of morphological primitivity is established of Tachyglossidae relative to Ornithorhynchus, and of monotremes as a whole relative to therians. It appeared that the composition of forelimb muscles in Tachyglossidae is more primitive above the elbow, while that in Ornithorhynchus is more primitive below the elbow. Some muscles, e.g. the $m$. serratus ventralis cervicis and the $m$. flexor digitorum profundus, are built in monotremes almost as primitive as in amphibians. In general, the forelimb of monotremes is confirmed to be a good model of premammalian locomotorium.
\end{abstract}

KEY WORDS: Monotremata, Zaglossus, forelimb, myology, muscle homology.

Peter P. Gambaryan [gambarpp@yandex.ru], Zoological Institute, Russian Academy of Sciences, Universitetskaya nab. 1, St. Petersburg, 199034, Russia; Alexander N. Kuznetsov [sasakuzn@mail.ru] \& Sergey V. Gerasimov, Department of Vertebrate Zoology, Faculty of Biology, Lomonosov Moscow State University, 1-12 Leninskie Gory, Moscow, 119234, Russia; Alexandra A. Panyutina, Severtsov Institute of Ecology and Evolution, Russian, Academy of Sciences, Leninsky av. 33, Moscow, 119071, Russia.

\section{Миология плечевого пояса и передней конечности современных Monotremata}

\author{
П.П. Гамбарян, А.Н. Кузнецов, А.А. Панютина, С.В. Герасимов
}

\begin{abstract}
РЕЗЮМЕ. Впервые проведен сравнительно-миологический анализ передних конечностей всех ныне живущих родов однопроходных млекопитающих. На основании детальной препаровки установлен уровень морфологической примитивности ехидновых в сравнении с утконосом и однопроходных в целом по сравнению с териевыми млекопитающими. Оказалось, что организация мускулатуры передней конечности у ехидновых примитивнее, чем у утконоса, в проксимальных отделах (выше локтя), а у утконоса она примитивнее в дистальных отделах (ниже локтя). Некоторые мышцы, такие как $m$. serratus ventralis cervicis и $m$. flexor digitorum profundus, у однопроходных построены почти столь же примитивно, как у амфибий. В целом, передняя конечность однопроходных вполне может служить подходящей моделью для реконструкции опорно-двигательной системы предков млекопитающих.
\end{abstract}

КЛЮЧЕВЫЕ СЛОВА: Monotremata, Zaglossus, передняя конечность, миология, гомология мышц.

\section{Introduction}

Since the total anatomical description of Ornithorhynchus by Meckel (1826), many myological studies of Ornithorhynchus and Tachyglossus were published, but only one was devoted to Zaglossus (Allen, 1912), due to its rarity. Since then, the first summary of the previous myological studies of the monotreme forelimb was published by Howell (1937), and the second one by Jouffroy et al. (1971). After that, there have been dissected only one specimen of Tachyglossus (Walter, 1988) and two specimens of Ornithorhynchus (Diogo \& Abdala, 2010).

In 1937, Howell explained his attempt to redescribe the shoulder muscles of Ornithorhynchus by the following characteristic of preceding studies: "For more than 50 years the work of Westling on the anatomy of Echidna, and for 40, that of McKay on Ornithorhynchus, have been standard, and have been quoted far and wide. Both are excellent papers, indicating unusual care in dissecting, and the latter is further useful in that the author quotes extensively from previous reports on the subject. Westling's figures are excellent, but insufficiently diversified, while those of McKay are rather poor. If one desires purely topographical detail these papers are entirely adequate, but they are too old to reflect modern concepts of morphology, and presentday reports upon the anatomy of this group are highly desirable." Today, we can repeat these words to introduce our descriptive myological paper, in which all the three monotreme genera are studied together for the first time. All their forelimb muscles will be considered and compared. 
As far as possible, we will apply the myological nomenclature accepted for therians. In our opinion, the homologies between therians and monotremes are more straightforward than they are used to be thought of since Howell's innovations in 1937. Howell boosted the problem by destroying the obvious idea of Westling (1889), McKay (1894), and other predecessors, that the big muscle occupying the infraspinous fossa of the scapula in monotremes is the $m$. infraspinatus. Instead, Howell suggested that it is the $m$. teres minor and, inevitably, came to difficulties in recognition of surrounding muscles and was obliged to introduce many new muscular names and concluded that monotremes are extremely aberrant mammals and cannot be used in deducing the primitive mammalian arrangement, contrary to general assumption of his time (Howell, 1936). Here, we make a step back towards Westling and McKay and towards the assumption, refuted by Howell, that monotremes, and especially Zaglossus, are a good model of primitive mammalian locomotorium.

Here, a special comment is necessary to clarify the concept of morphological primitivity. Today, the statement, that some morphological condition is primitive or advanced, is often regarded as synonymic with the statement, that it is plesiomorphic or apomorphic in phylogenetic sense. If this was true, any decision on primitivity would be impossible and senseless beyond phylogenetic analysis. However, evolution proved to be mosaic, which means that there is never a total progress or regress of all features in a unison. In representatives of crown-groups, different old-fashioned features coexist for a long time, as anachronisms, with highly upgraded features. More than that, ancestral features may be recruited again and again by means of pedomorphoses or fixation of atavisms. That is why, there was never and will not be constructed a phylogenetic tree which accommodates all known features of involved organisms, and consensus trees are created instead. And that is why, tree-independent criteria are necessary to estimate the balance of progress and regress in each particular phylogenetic lineage. Such criteria were known to morphologists long before the birth of cladistics and phylogenetics, and they still persist in reduced form in the procedure of estimation of character polarity. "Primitive" generally means simple, homonomic, undifferentiated, widespread at the lower level of organization, etc. Indeed, the morphological primitivity is often very clear. From this standpoint, whenever possible, we will try to qualify conditions of particular muscles in different monotreme genera as primitive or advanced. In this way, we hope to help future researchers to treat properly the biological sense of morphological evolution of mammals and mammaliforms, when, very soon, their molecular phylogeny is finally built.

\section{Materials}

Zaglossus bruijnii (Peters et Doria, 1876) - collection of Zoological Museum of Moscow University: adult male ZMMU MS579A (alcohol), adult ZMMU MS579B (formalin), 1 uncatalogued adult female (alcohol).

Tachyglossus aculeatus (Shaw, 1792) — private collection: 2 adult specimens ( 1 - formalin, 1 alcohol).

Ornithorhynchus anatinus (Shaw, 1799) — private collection: 1 specimen retaining teeth (alcohol).

\section{Methods}

Each muscle will be described in the same order: general comments (if necessary), origin, insertion, and final remarks (if necessary). Since the main subject of our study is Zaglossus, the descriptions of origin and insertion will start with Zaglossus, followed by Tachyglossus, and, finally, Ornithorhynchus. Correspondingly, on the Figures, Zaglossus is designated by 'A', Tachyglossus by 'B', and Ornithorhynchus by ' $\mathrm{C}$ '.

Most of the drawings show the left limb, although some of them were initially drawn from the right limb and then flipped for the sake of visual consistency. Right side is only shown on the dorsal and ventral views of the whole body in order to fit the superficial and deeper muscular layers on the same figure, one on the left and the other on the right.

The osteological structures of the forelimb, which are important in myology, are shown for Zaglossus on Figs. 1-4. Only the humerus (Fig. 2) is represented by photos, while the shoulder girdle, antebrachium, and manus are represented by X-ray CT images (Figs, 1, 3, and 4 , respectively) obtained from scans of the whole animal before dissection; this is necessary to show the included bones in their natural articulation. Note, that the big compex-shaped preaxial bone in the proximal carpal row is formed by three ossifications, which Holmgren (1952) termed radiale, centrale 1, and centrale 3 . Keepeng more common terminology, we call this bone radiale+intermedium +centrale (= scaphocentralolunatum of some therians, such as colugos and bats (Panyutina et al., 2015)).

On the drawings of attachments (Figs. 19-23), the origins are simply filled with colours, and the insertions are, in addition, highlighted by cross-hatching. Each muscle has its own unique colour, as well as portions of some complex muscles, e.g., the $m$. serratus ventralis cervicis and the $m$. flexor digitorum profundus.

The abbreviations used on figures are as follows:

c. - caput

dig. - digitus

ext. - extensor

fl. - flexor

fl. dig. pr. - flexor digitorum profundus

lig. - ligamentum

m., mm. - musculus, musculi

n. - nervus

r. - ramus

s. v. - serratus ventralis

For cervical vertebrae and digits we use Roman 


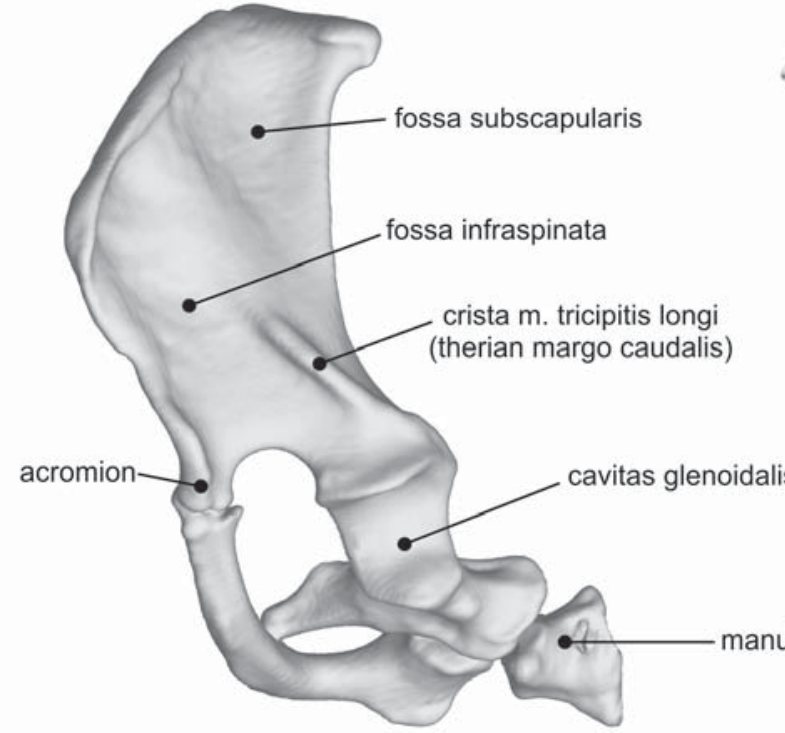

A1

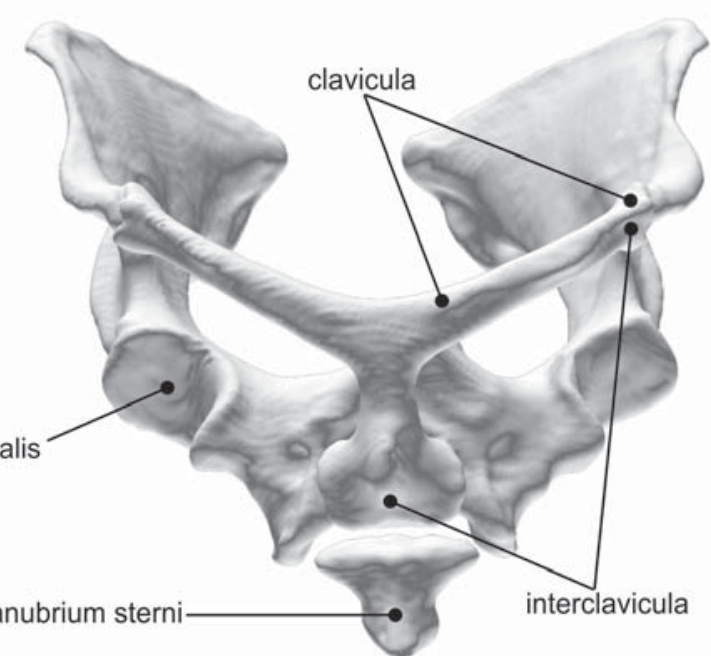

A2

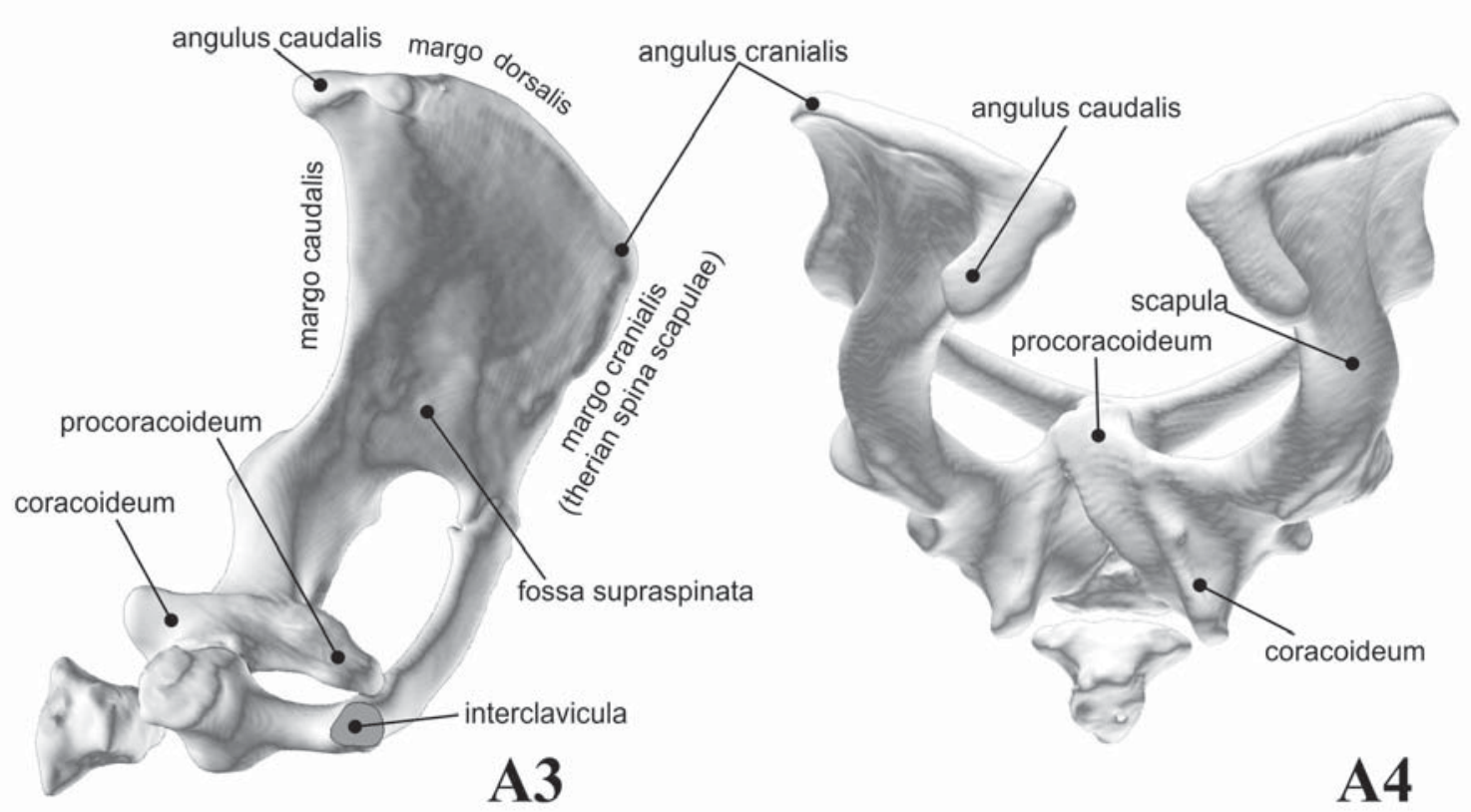

Figure 1. X-ray CT images of the shoulder girdle of Zaglossus. 1 - lateral outer view, 2 - ventral outer view, 3 - medial inner view, 4 - dorsal inner view. On 1 and 3, only the left half of the girdle is shown. Note the non-therian disposition of the muscular fossae on the scapula.

numerals, and for other regions of the vertebral column, for ribs and phalanges we use Arabic numerals.

\section{Muscles of shoulder girdle}

Musculus sternomastoideus (Figs. 6, 7).

Origin. In Zaglossus, the muscle originates by a narrow aponeurosis from a special longitudinal crest at the caudal margin of the squamosum.

In Tachyglossus and Ornithorhynchus, the muscle originates by a short flat tendon from the point of transition of the base of the zygomatic arch into transverse crest of the skull and, also, from the aponeurosis of origin of the $m$. masseter.

Insertion (Fig. 19). In Zaglossus, the muscle inserts over the $m$. pectoralis on the caudal two-thirds of the stem of the interclavicle and along the entire sternum, except for its xiphoid process.

In Tachyglossus, it inserts on the manubrium and first two segments of the sternum.

In Ornithorhynchus, it inserts on the medial onethird of the anterior border of the clavicle, except for its medial tip. 

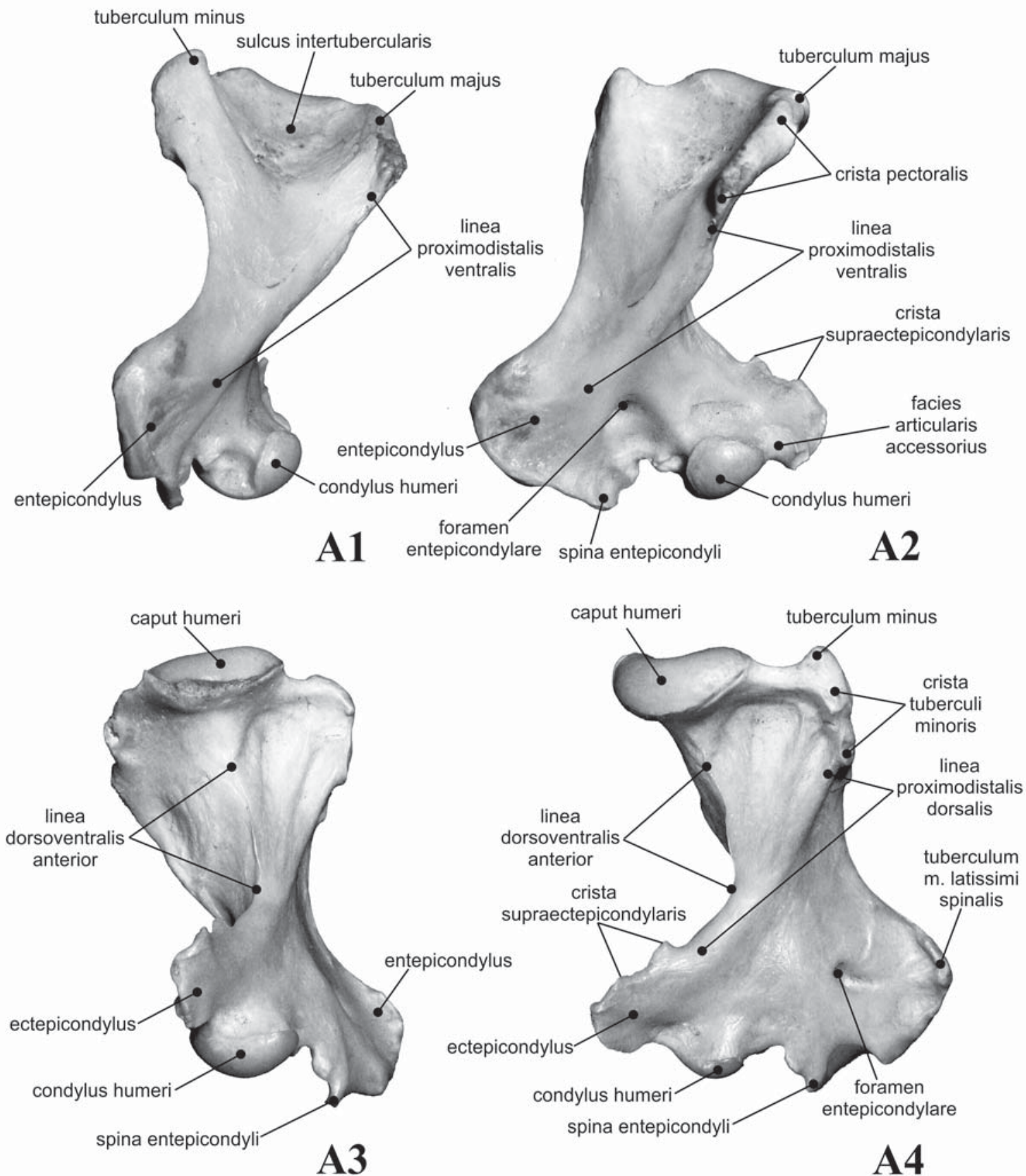

Figure 2. Photos of the left humerus of Zaglossus. 1 - proximal ventral surface, 2 - distal ventral surface, 3 - proximal dorsal surface, 4 - distal dorsal surface. The surfaces, as well as the linea proximodistalis ventralis, linea proximodistalis dorsalis, and linea dorsoventralis anterior, are designated according to Romer (1922). The last line, represented by a tiny ridge, separates on the proximal dorsal surface the areas of the $m$. brachialis (which is preaxial to this line) and $m$. triceps accessorius (postaxial to it). The crista pectoralis is equivalent of the crista tuberculi majoris. Note the absence of the articular trochlea humeri for ulna; instead, there is a single spherical condyle for both radius and ulna, supplied with an accessory facet at the radial side. 


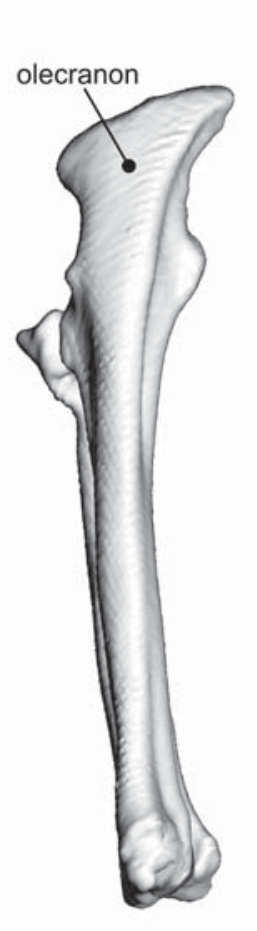

A1

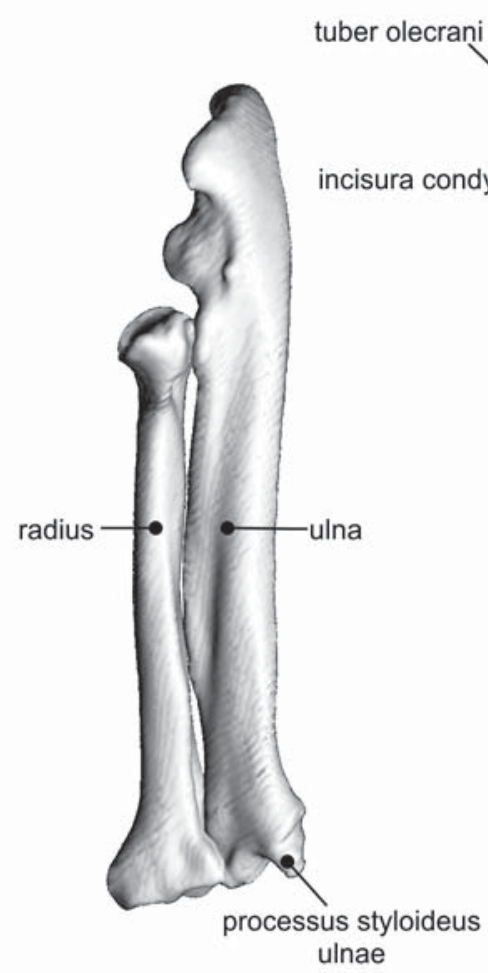

A2

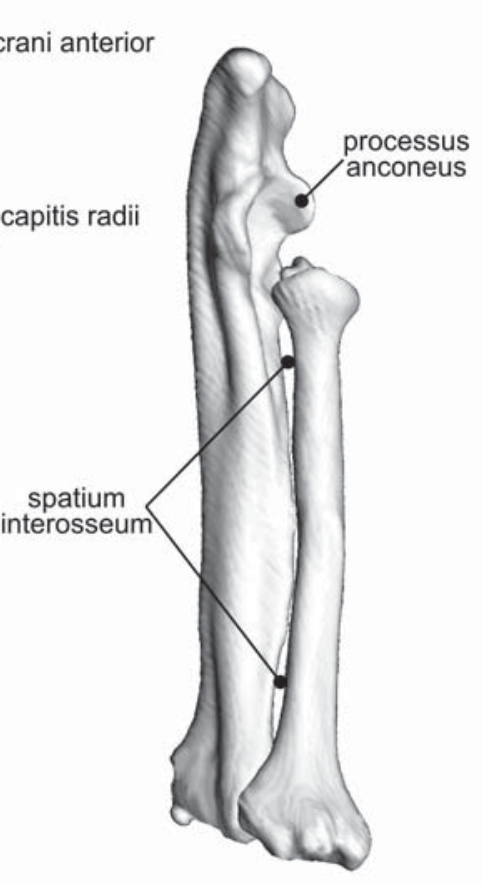

A4

Figure 3. X-ray CT images of the left antebrachium of Zaglossus. 1 - postaxial side, 2 - anterior side, 3 - preaxial side, 4 posterior side.

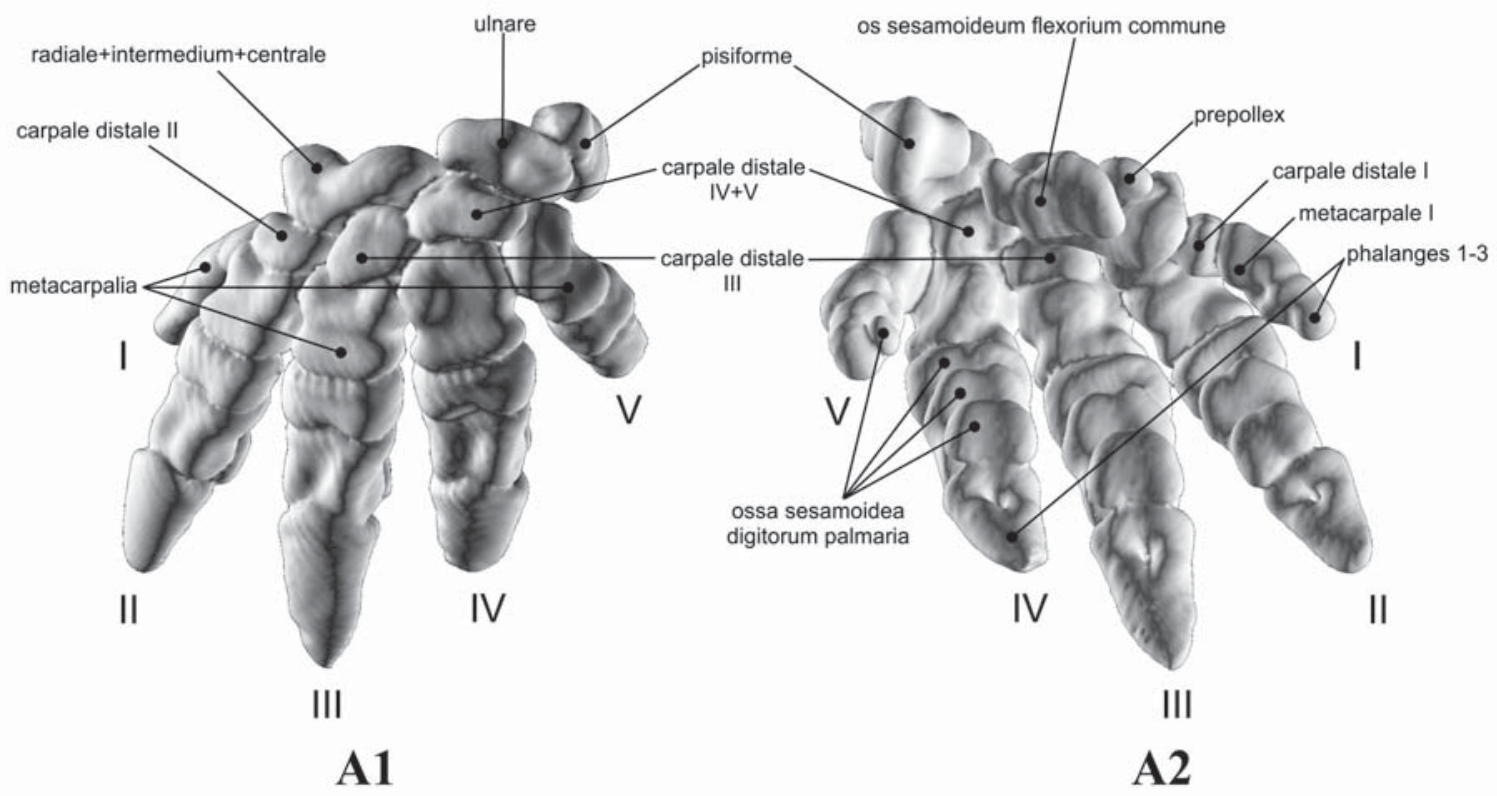

Figure 4. X-ray CT images of the left manus of Zaglossus. 1 - dorsal view, 2 - palmar view. 

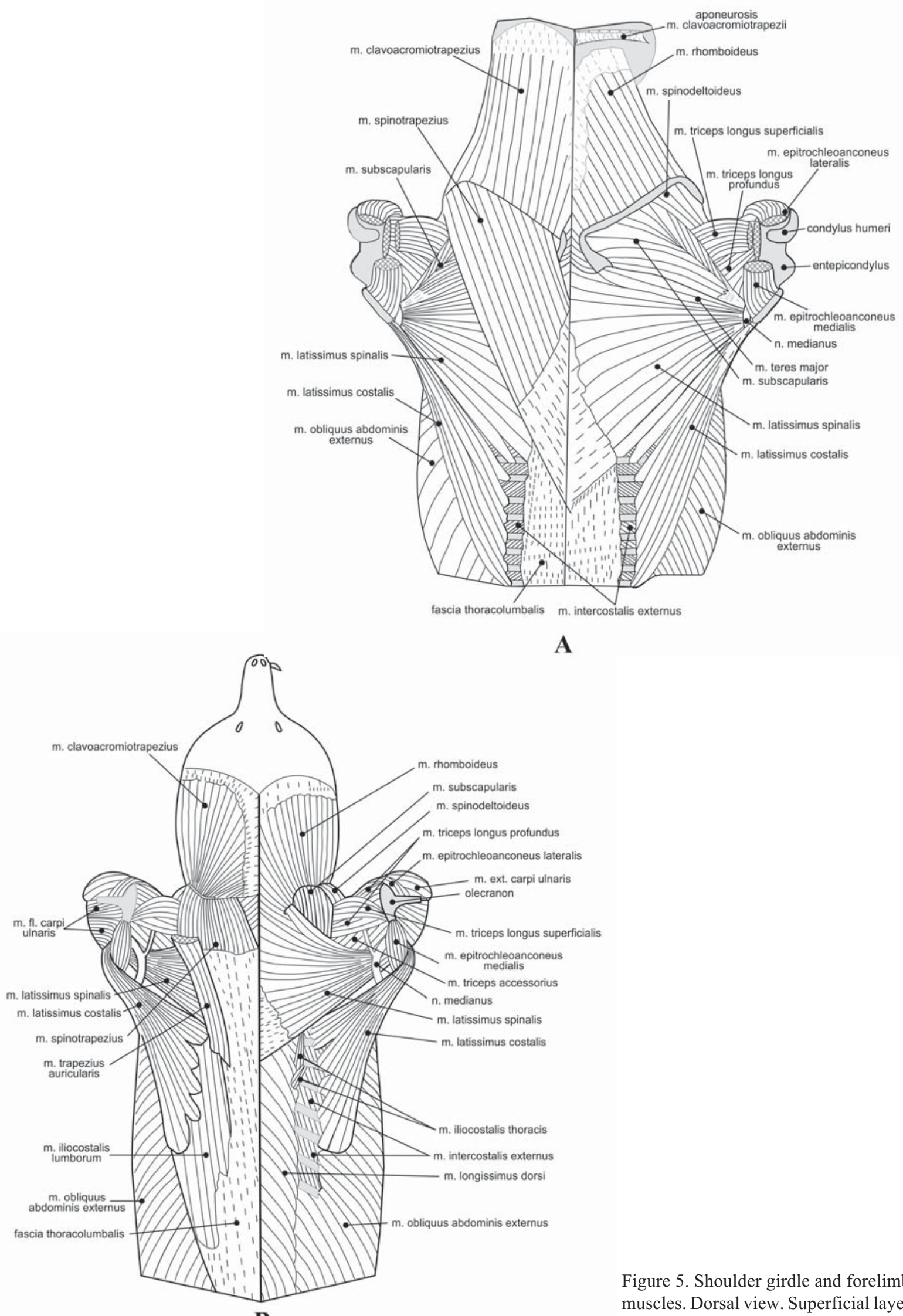

Figure 5. Shoulder girdle and forelimb muscles. Dorsal view. Superficial layer B at the left, deeper layer at the right. 
Figure 5 (continued).

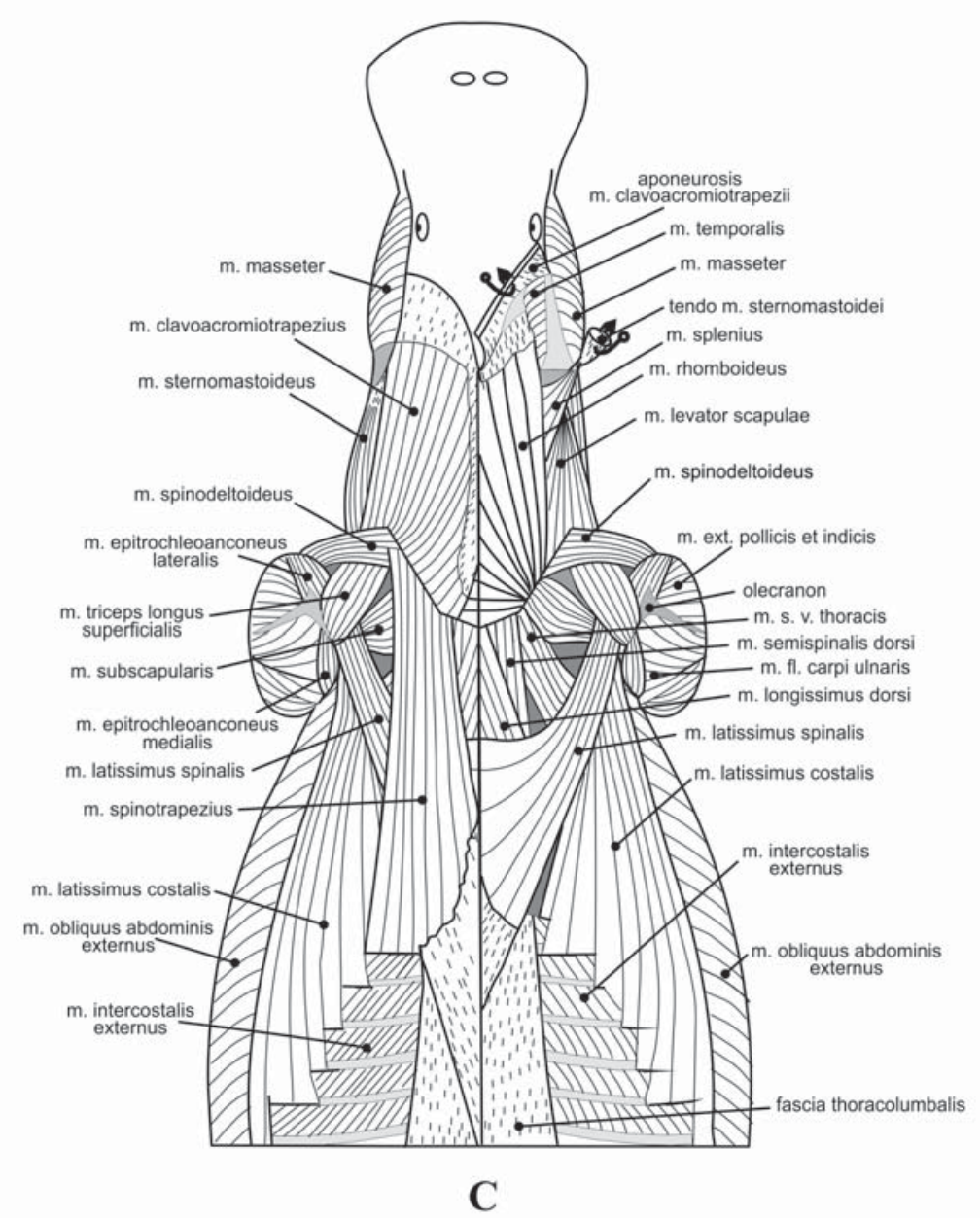

Musculus cleidomastoideus (Figs. 6C, 7C).

The muscle is absent in Tachyglossidae.

Origin. In Ornithorhynchus, the muscle originates by a short tendon from the caudolateral area of the skull, passing from below the ventral edge of the $m$. splenius capitis (on the contrary, the m. sternomastoideus, originates above the $m$. splenius capitis).

Insertion (Fig. 19C). In Ornithorhynchus, it inserts in the middle of the anterior border of the clavicle.

Musculus clavoacromiotrapezius (Figs. 5-7).

The $m$. clavotrapezius and the $m$. acromiotrapezius are not divided in monotremes comprising a single muscle, but its cephalic portion (corresponding to the $m$. clavotrapezius) is significantly thicker than the cervical one (corresponding to the $m$. acromiotrapezius).

Origin. In Zaglossus, the muscle originates by aponeurosis from the transverse crest of the occiput (when approaching the midline, the aponeurosis increases in length) and from the dorsal midline of the anterior part of the neck up to the level of the posterior end of the neural spine of the epistropheus (here, the aponeurosis decreases in length again). Note, that the transverse crest of the skull is curved, passing, at first, dorsorostrally from the point of origin of the m. sternomastoi- deus, between the base of the zygomatic arch and occiput and, then, medially (transversely) over the parietale and up to the middle of the supraoccipitale.

In Tachyglossus, the muscle originates by aponeurosis from the transverse crest of the parietale (when approaching the midline, the aponeurosis increases in length) and from the dorsal midline of the neck up to the level of the last cervical vertebra (here, the aponeurosis decreases in length again).

In Ornithorhynchus, the muscle originates by aponeurosis from the transverse crest of the parietale and interparietale (when approaching the midline, the aponeurosis decreases in length) and from the dorsal midline of the anterior two-thirds of the neck (here, the aponeurosis increases in length again).

Insertion (Figs. 19, 20). In Zaglossus, the muscle inserts by aponeurosis on lateral (distal) one-sixth of the clavicle, acromion, cranial border of the scapula (just caudal to the insertion of the $m$. atlantoscapularis), and on the cranial-most part of the dorsal border of the scapula; the aponeurosis is thin here and fused with the end of the $m$. spinotrapezius.

In Tachyglossus, the muscle insertion varies. In one specimen, we found it on the lateral end of the clavicle, acromion, cranial border of the scapula, and its dorsal 


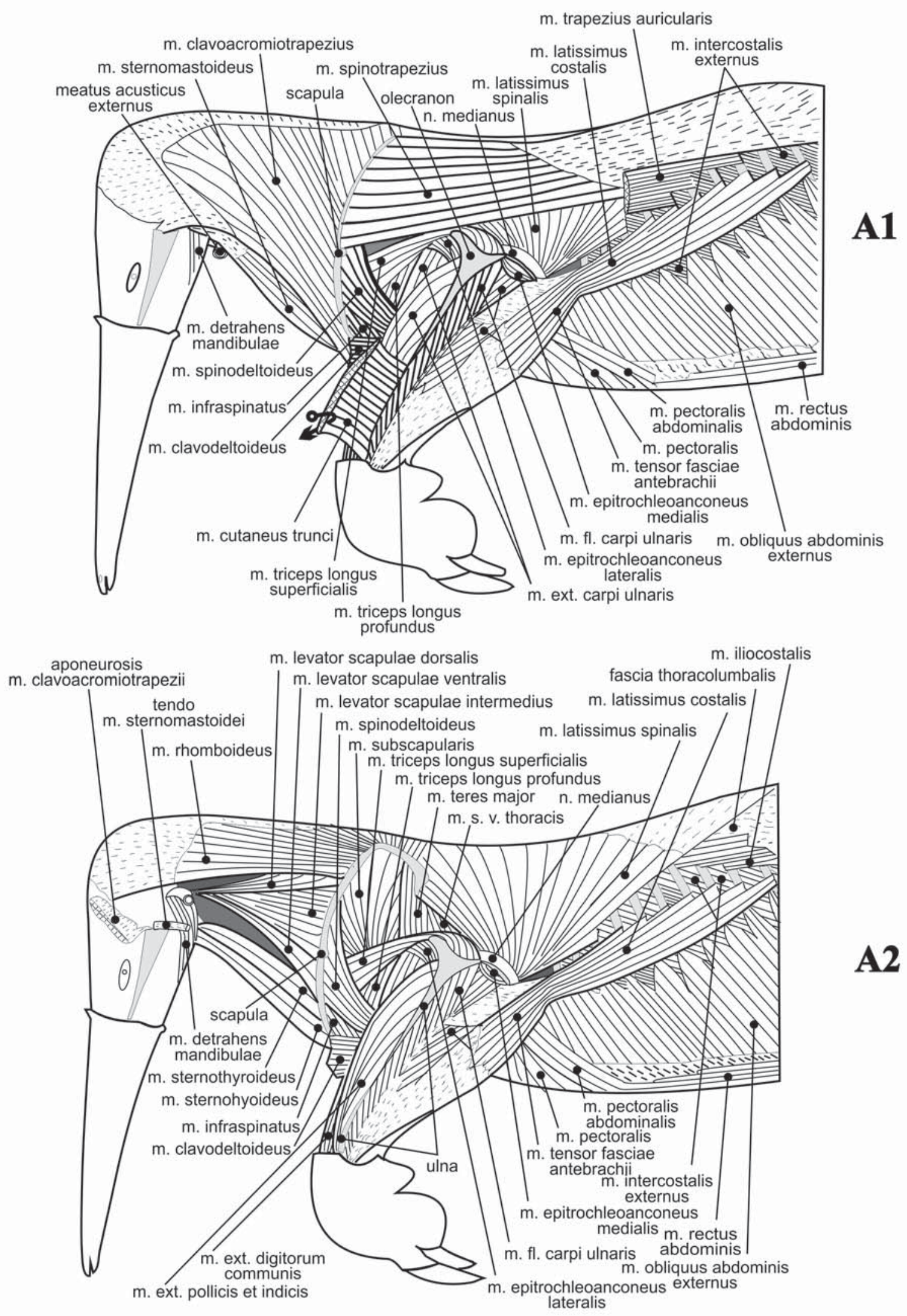



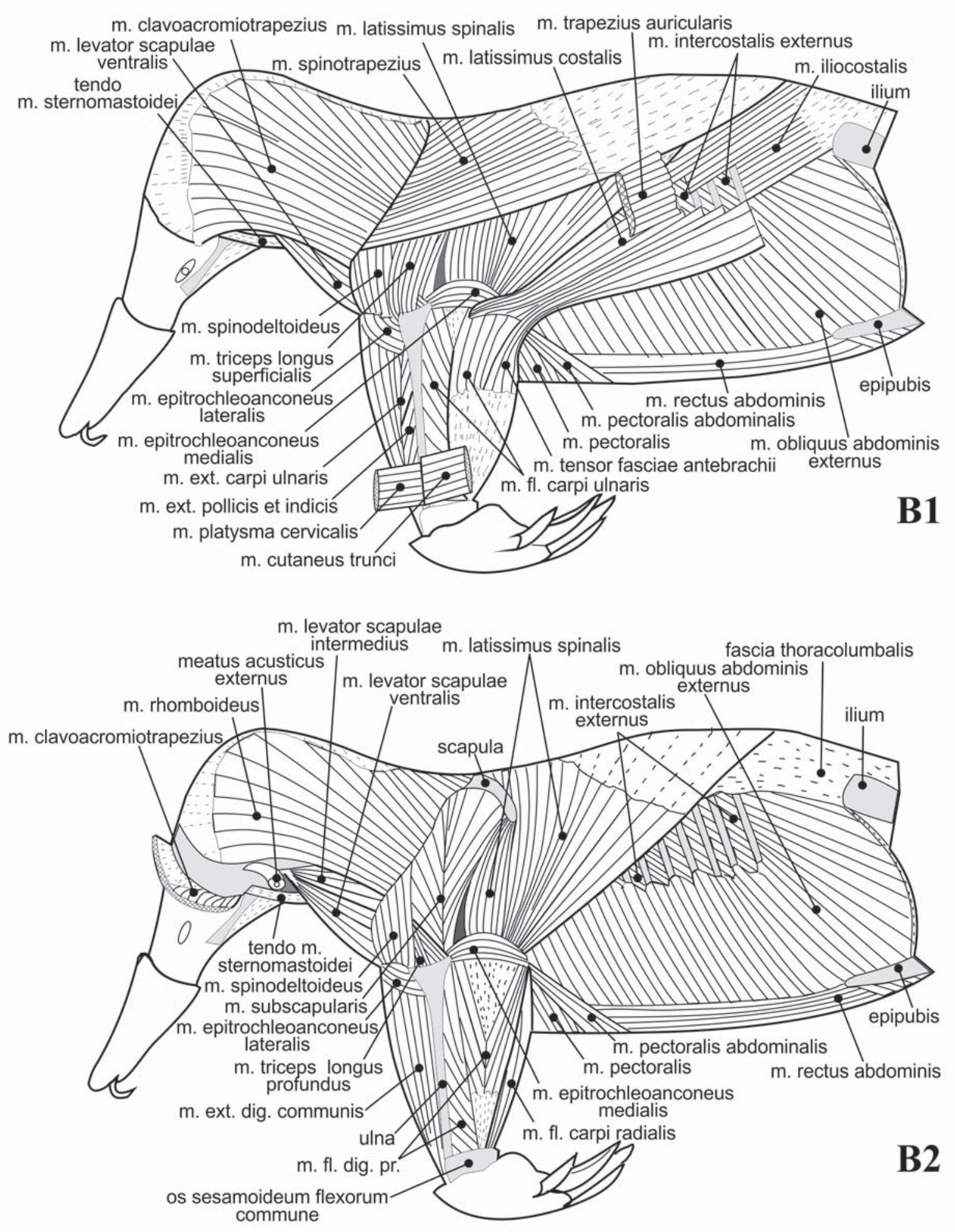

Figure 6. Shoulder girdle and forelimb muscles. Lateral view. 1 - superficial layer, 2 - deeper layer, C3 — the deepest layer (antebrachium removed) in Ornithorhynchus.

Continued on pages 10-11. 


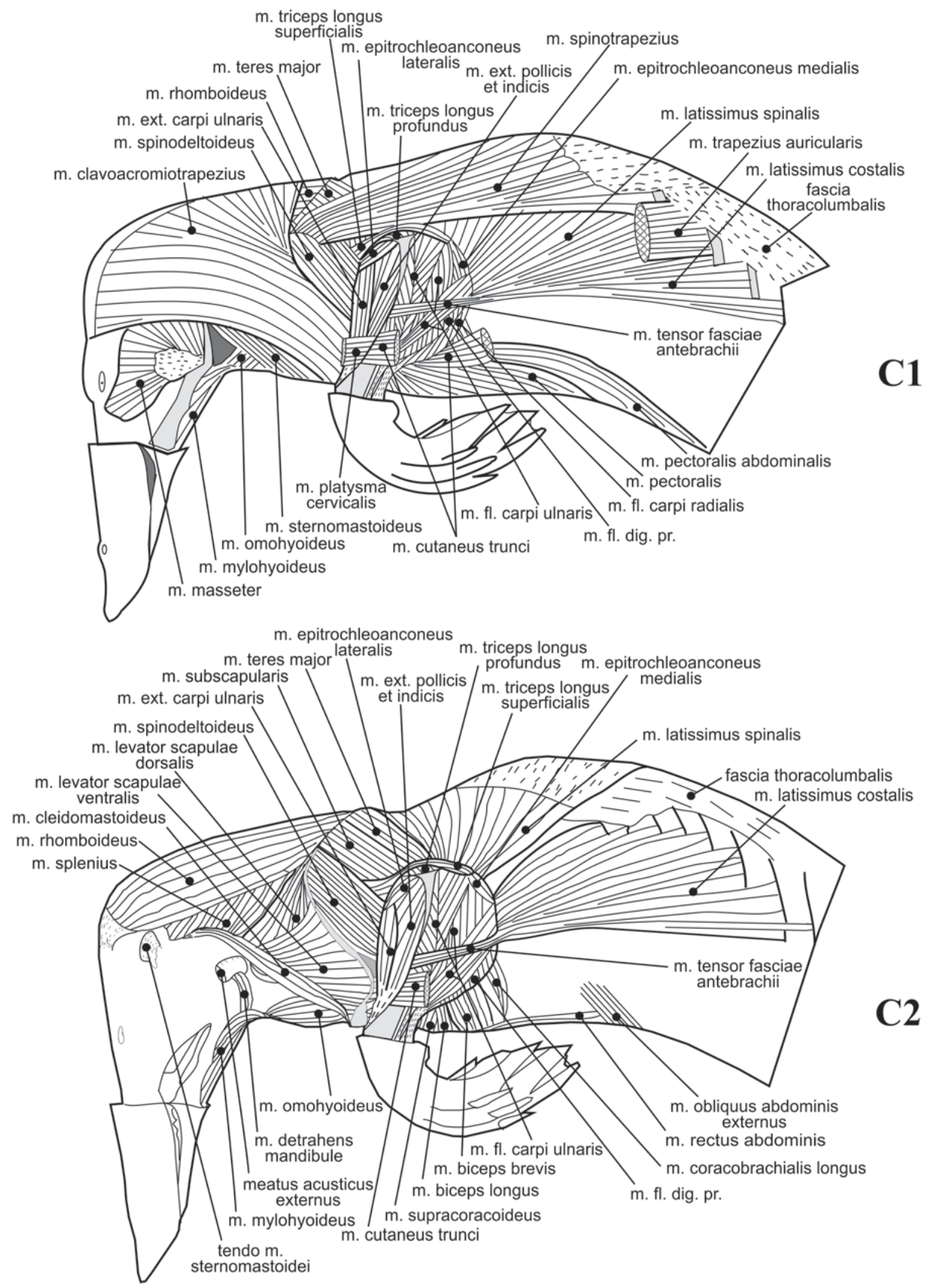

Figure 6 (continued). 


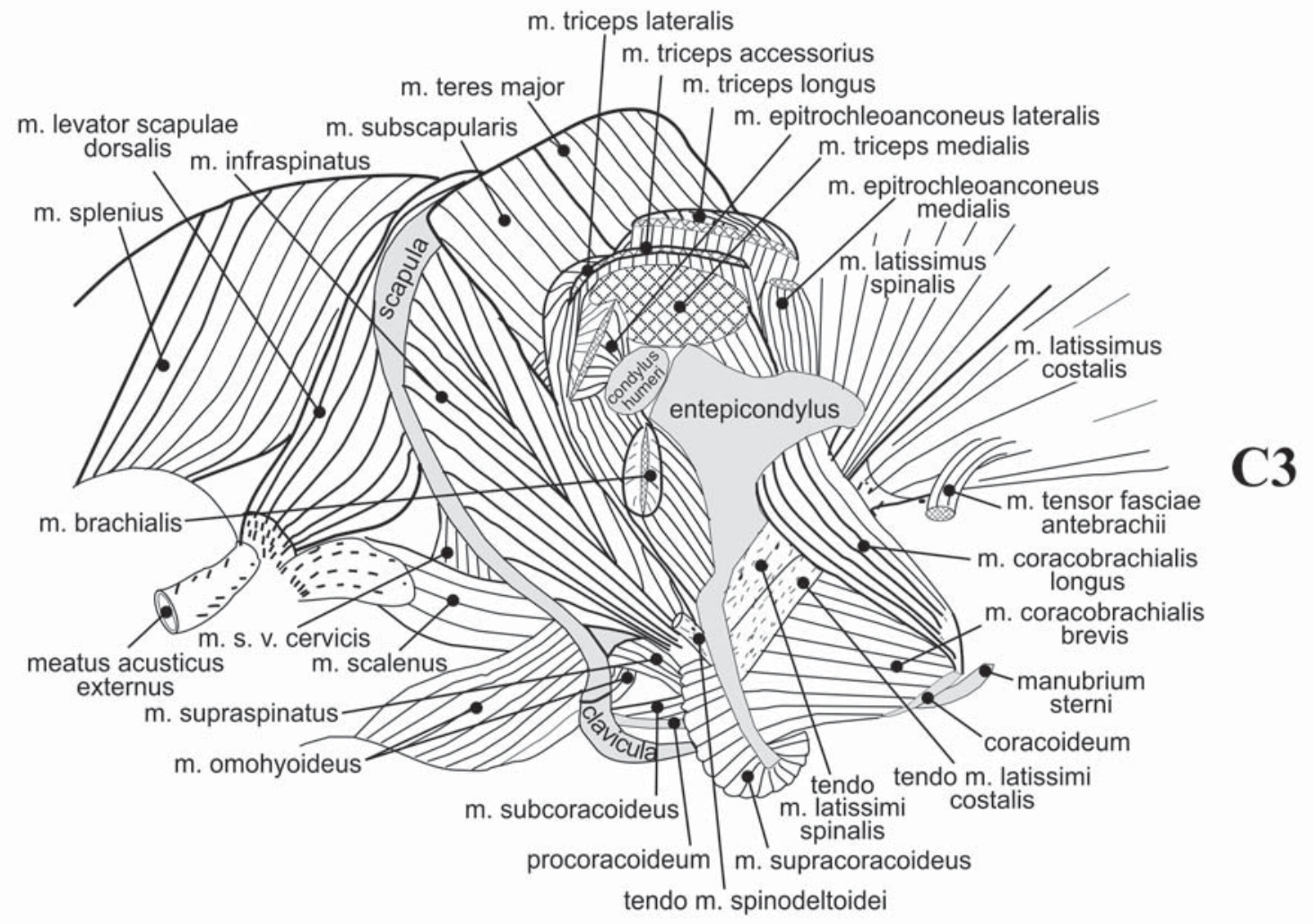

Figure 6 (continued).

border, where it is fused with the $m$. spinotrapezius (this corresponds to the earlier descriptions by Westling (1890) and Walter (1988)). In the other specimen, the insertion was restricted to two separate areas, which are the dorsal half of the cranial border of the scapula and the middle three-fifths of its dorsal border; so, in contrast to the first specimen, the muscle did not insert on the clavicle, acromion, and craniodorsal angle (angulus cranialis) of the scapula.

In Ornithorhynchus, the muscle inserts on the lateral end of the clavicle and the entire cranial border of the scapula.

Remarks. The cervical portion of this muscle (corresponding to the therian $m$. acromiotrapezius), as well as the $m$. levator scapulae, definitely mark, by their insertion, the cranial border of the scapula (Romer, 1922). This association proves the homology of this border in monotremes and the scapular spine in therians, in spite of the non-endochondral ossification of the spine (Sánchez-Villagra \& Maier, 2002).

Musculus spinotrapezius (Figs. 5, 6).

Origin. In Zaglossus, the muscle originates by aponeurosis from the neural spines of thoraco-lumbar vertebrae; in one specimen, from the 3rd through 16th thoracic vertebrae; in two specimens, from the 7 th thoracic vertebra up to the 2nd lumbar vertebra (according to Allen, 1912, the origin starts from the 10th thoracic vertebra). In addition, some caudal fibres of this muscle originate together with the $m$. trapezius auricularis by tendons attached to ribs 10-13 ventral to the epaxial muscles (spine extensors). The cranial-most tendon is fused to its rib much stronger than the other three, through almost one-fifth of the length of the 10th rib in its middle part.

In Tachyglossus, the muscle originates fleshy from the neural spine of the 8th thoracic vertebra and by aponeurosis from the neural spines of all subsequent thoracic vertebrae. In contrast to Walter (1988), we have not recorded origin from the ribs.

In Ornithorhynchus, the muscle originates by aponeurosis from the neural spines of thoracic vertebrae 713 and from the dorsal half of the 9th rib, distal to its tubercle.

Insertion (Fig. 20). In Zaglossus, the muscle inserts along the dorsal one-fifth of the cranial border of the scapula, its craniodorsal angle and the cranial twothirds of its dorsal border. Insertion is positioned somewhat apart from the border itself on the external surface of the scapular blade.

In Tachyglossus, the muscle inserts on the middle three-fifths of the dorsal border of the scapula.

In Ornithorhynchus, the muscle inserts on the dorsal one-fifth of the cranial border of the scapula, its craniodorsal angle and the cranial one-fifth of its dorsal border. At the cranial border of the scapula, the inser- 
tion of this muscle wedges into the origin of the $m$. spinodeltoideus, dividing it into two portions, of which the posterior one comes out onto the external surface of shoulder musculature from under the $m$. spinotrapezius, while the anterior portion completely overlies it.

Remarks. In all monotremes, the aponeurosis of origin gradually increases in length in antero-posterior direction, and that is why the muscular fibres do not.

Musculus trapezius auricularis (Figs. 5, 6).

Origin. In Zaglossus, the muscle originates as slips, paralleling the $m$. spinotrapezius, from ribs 8-10 and the superficial fascia of the $m$. iliocostalis. In one of the three specimens examined, there was an additional (about 6 times less massive) posterior portion originating at the level of ribs 11-14 from the external surface of the $m$. latissimus costalis; it joined the major portion close to the origin.

In Tachyglossus, the muscle originates as slips from ribs 11 (almost at its mid-height) and 12, and, more posteriorly, from the external surface of the aponeurosis of origin of the $m$. spinotrapezius, approaching here the level of the spine extensors.

In Ornithorhynchus, the muscle originates as two slips from the dorsal one-third of rib 10 and from the middle of rib 11.

Insertion. In Zaglossus, the most anterior slip inserts on the top of the auricle and the rest of the muscle fuses into the internal surface of the $m$. platysma cervicalis.

In Tachyglossus, the muscle fuses into the m. platysma cervicalis short of reaching the head.

In Ornithorhynchus, the slip from rib 10 reaches the auricle and the slip from rib 11 fuses into the $m$. platysma cervicalis.

Musculus rhomboideus (Figs. 5, 6).

Tachyglossidae show incipient division of the muscle into two portions with sharply different fibre direction; the cranial portion, corresponding to the therian $m$. rhomboideus capitis, passes anteroposteriorly, while the caudal one, corresponding to the therian $\mathrm{m}$. rhomboideus thoracis, runs more or less mediolaterally. In Ornithorhynchus, the muscle shows no such division.

Origin. In Tachyglossidae, the muscle originates from the transverse crest of the occiput and the dorsal midline of the whole neck. From the transverse crest and up to the neural spine of the epistropheus inclusive, the origin is aponeurotic, while more posteriorly, the fibres reach the midline to meet those of the contralateral muscle above the nuchal ligament (shaped as a sagittal fascia giving rise to the $m$. semispinalis capitis and the $m$. splenius).

In Ornithorhynchus, the origin is similar, but at the transverse crest of the occiput, particularly in its lateral part, it is positioned further apart (more posteriorly) from the origin of the m. clavoacromiotrapezius than in Tachyglossidae.

Insertion (Fig. 20). In Tachyglossidae, the cranial portion of the muscle (=m. rhomboideus capitis), in- serts on the uppermost part of the cranial border of the scapula (under the $m$. spinotrapezius), its craniodorsal angle, and the internal and external sides of its dorsal border, while the posterior portion $(=m$. rhomboideus thoracis), inserts on the internal side of the dorsocaudal angle (angulus caudalis) of the scapula.

In Ornithorhynchus, the muscle inserts on the internal side of the caudal part of the dorsal border of the scapula up to its dorsocaudal angle.

Musculus levator scapulae $(=m$. omotransversarius) (Figs. 6-8) is divided into three closely associated portions: the $m$. levator scapulae dorsalis, $m$. levator scapulae intermedius, and m. levator scapulae ventralis $(=m$. levator claviculae of Diogo \& Abdala, 2010).

Origin. In Zaglossus, the m. levator scapulae dorsalis originates from the ventral side of the occiput just posterior to the $m$. detrahens mandibulae and somewhat medial to the $m$. rectus capitis lateralis by a rather wide aponeurosis, which, having passed onto the neck close to the capsule of the atlantooccipital joint, fuses with the tendon of origin of the subsequent portion. The origin of the m. levator scapulae intermedius extends from the capsule of the atlantooccipital joint posteriorly, onto the ventral border of the epistropheal rib, where it almost fuses with the origin of the subsequent portion. The $m$. levator scapulae ventralis originates from the ventral border of the epistropheal rib.

In Tachyglossus, the $m$. levator scapulae dorsalis originates from the ventral side of the occiput just posterior to the $m$. detrahens mandibulae and somewhat medial to the $m$. rectus capitis lateralis by a tendon, which, having passed close to the capsule of the atlantooccipital joint, converges with the common tendon of origin of the two other portions arising from the ventral tubercle of the atlas wing. From the point of convergence of the two tendons, muscular fibres begin. In addition, the $m$. levator scapulae intermedius and the $m$. levator scapulae ventralis originate from the ventral border of the epistropheal rib - the m. levator scapulae intermedius has here an accessory tendon of origin, and the $m$. levator scapulae ventralis originates fleshy throughout the rib length.

In Ornithorhynchus, all the three portions originate mainly from the atlas, at the ventral tubercle of its wing. The $m$. levator scapulae dorsalis originates here by a very short flat tendon, the $m$. levator scapulae intermedius by a longer tendon, and the $m$. levator scapulae ventralis by an aponeurosis being separated from the previous two by the $m$. scalenus. In addition, the $m$. levator scapulae intermedius has a thin accessory tendon arising from the articular capsule of the atlantooccipital joint, and posterior fibres of the $m$. levator scapulae ventralis originate fleshy along the caudoventral border of the atlas wing and up to the epistropheal rib.

Insertion (Figs. 19, 20). In Zaglossus, the m. levator scapulae dorsalis inserts on the internal side of the dorsal border of the scapula, except for the cranial onetenth and caudal one-fourth of its length. The insertion of the $m$. levator scapulae intermedius starts just crani- 


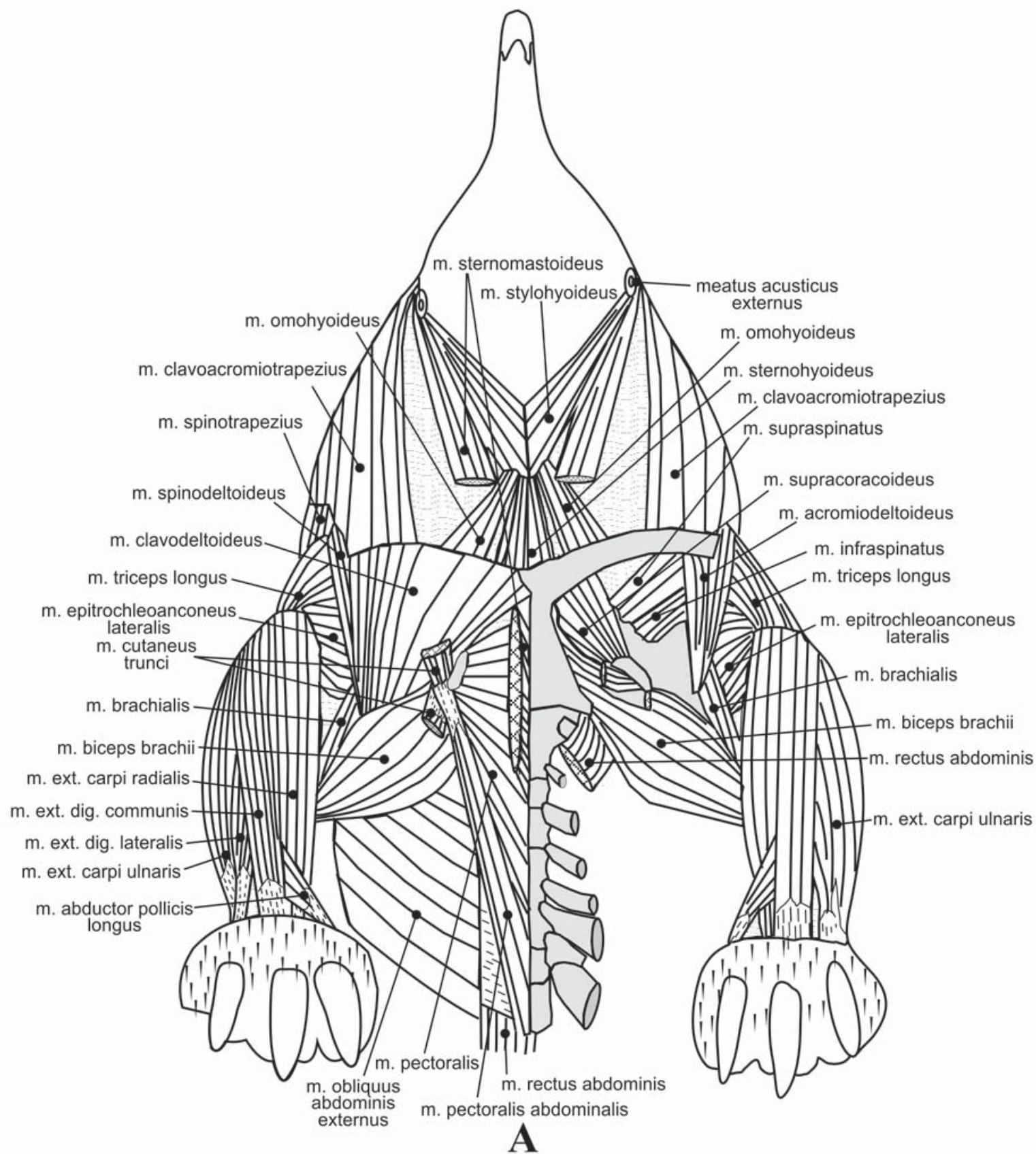

Figure 7. Shoulder girdle muscles and forelimb muscles. Ventral view. Superficial layer at the left, deeper layer at the right, $\mathrm{C} 2$ - the third, deepest layer in Ornithorhynchus.

al to that of the previous portion; it occupies the internal side of the remaining cranial part of the dorsal border of the scapula and the dorsal half of its cranial border. The $m$. levator scapulae ventralis inserts just ventral to the previous portion on the remaining part of the anterior border of the scapula, acromial process and the distal one-sixth of the clavicle.

In Tachyglossus, the $m$. levator scapulae dorsalis inserts on the internal side of almost the entire dorsal border of the scapula and dorsal one-fifth of its cranial border. The $m$. levator scapulae intermedius inserts on the medial side (in contrast to Zaglossus, the insertion does not come out onto the external side) of the subsequent two-fifths of the cranial border, directly adjoining the previous portion or (in one specimen) somewhat apart from it. The $m$. levator scapulae ventralis inserts on the internal side of the remaining ventral two-fifths of the cranial border of the scapula and acromion.

In Ornithorhynchus, the m. levator scapulae dorsalis inserts on the internal side of the cranial three-fifths 


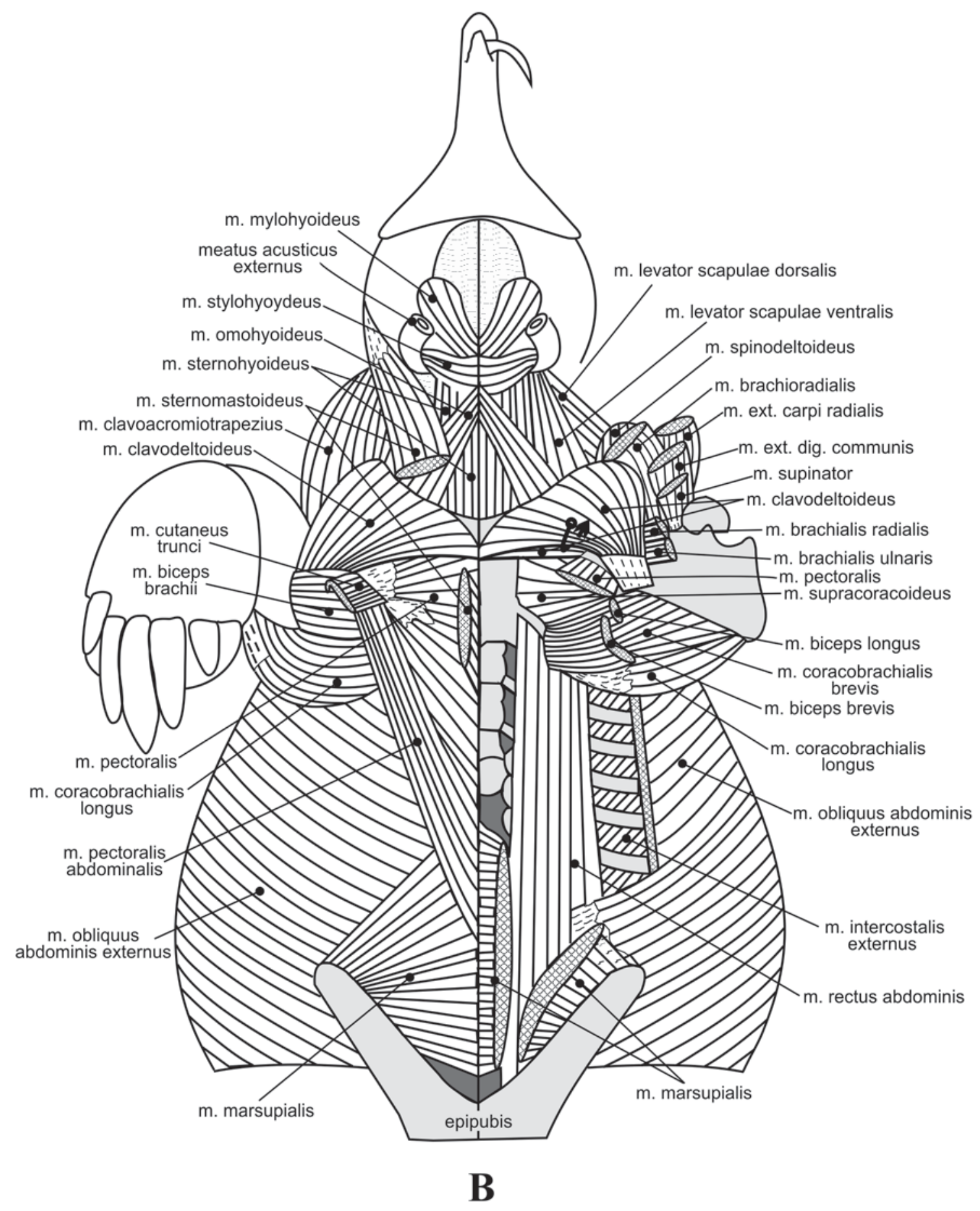

Figure 7 (continued).

of the dorsal border of the scapula and on the dorsal half of its cranial border. The $m$. levator scapulae intermedius inserts on a short segment of the cranial border of the scapula just below the previous portion and above the subsequent portion. The $m$. levator scapulae ventralis inserts on the remaining ventral part of the cranial border of the scapula, acromial process, and the distal one-fifth of the clavicle. Note, that the inser- tions of all the three portions cover both internal and external sides of the cranial border of the scapula.

Remarks. The $m$. levator scapulae dorsalis corresponds to the $m$. atlantoscapularis of therians, the $m$. levator scapulae ventralis is the $m$. atlantoacromialis, and the $m$. levator scapulae intermedius was apparently lost in therians. The wide and variable origin in monotremes shows that its association with the atlas in 


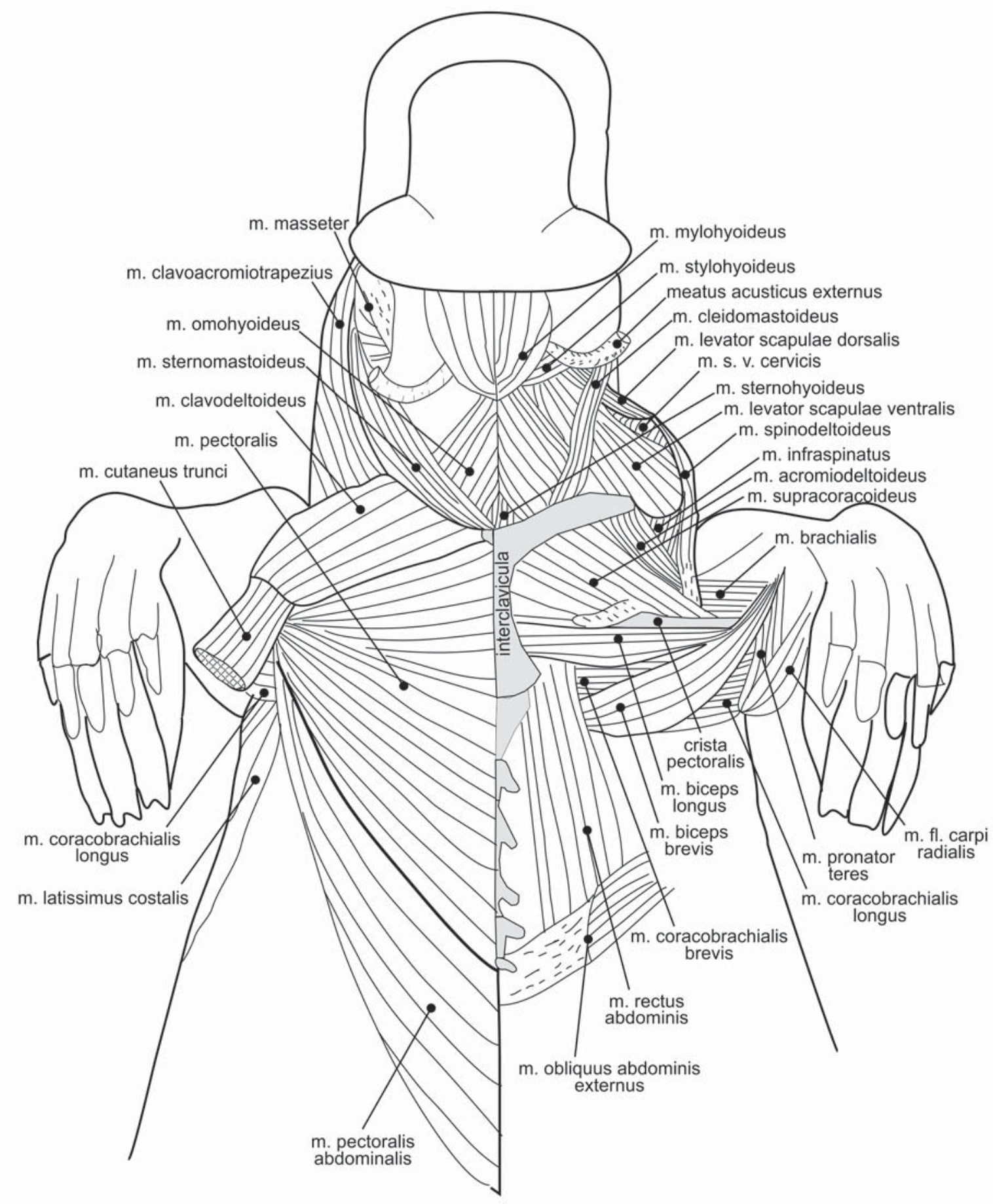

C1

Figure 7 (continued).

therians and Ornithorhynchus should be regarded as an advanced condition achieved independently. By insertion, especially in Tachyglossidae, the $m$. levator scapulae dorsalis looks like the anterior-most slip of the $m$. serratus ventralis cervicis. Indeed, the $m$. levator scapulae of man represents both of these muscles fused together
Musculus serratus ventralis cervicis $(=m$. serratus anterior profundus) (Figs. 7, 9, 11) in all monotremes consists of six slips associated, respectively, with cervical vertebrae II-VII (i.e., all, except for the atlas). The origins of the slips on the cervical ribs are compact and insertions on the internal side of the scapula are wide, so that each slip spreads towards the scapula as a flat 


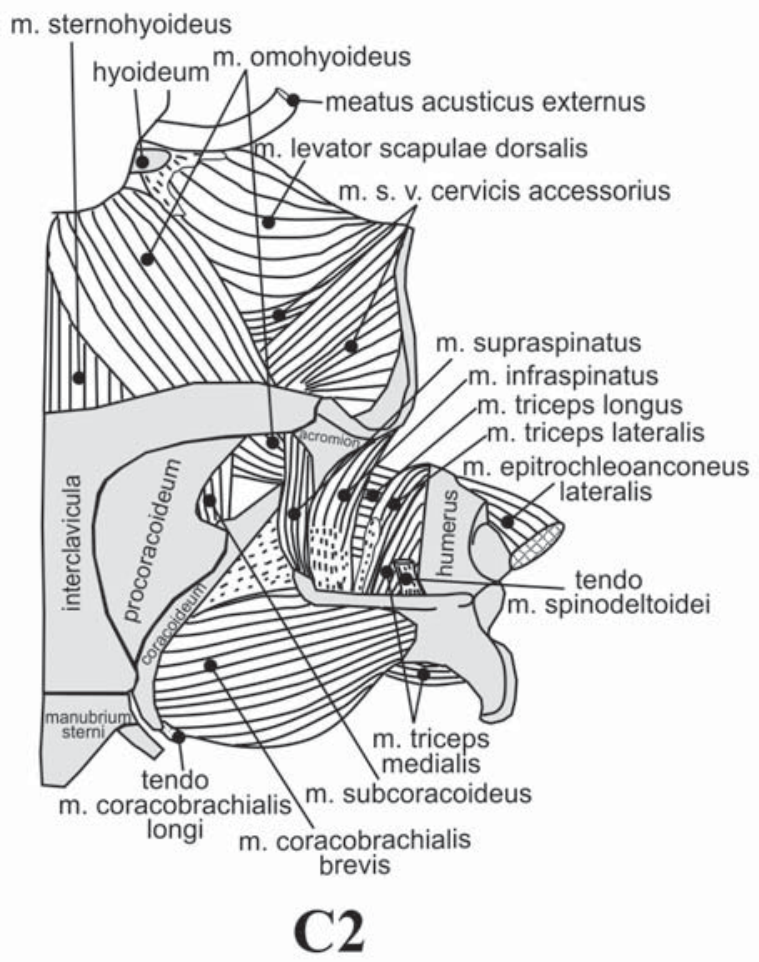

Figure 7 (continued).

fan. Each subsequent slip lies lateral to that from the preceding vertebra and, hence, closer to the scapula. The insertion of each subsequent slip is positioned lower on the internal side of the scapula than that of the preceding slip and expands further anteriorly and posteriorly, i.e., each next slip forms a wider fan. The slips from the last three cervical vertebrae of Tachyglossidae and the last five of Ornithorhynchus bifurcate (i.e., middle part of the fan is absent). In this case, we designate the caudal and cranial portions of the slip as main and accessory, respectively, or the $m$. serratus ventralis cervicis proprius and $m$. serratus ventralis cervicis accessorius. In general, the main portions pass from the vertebrae to the scapula subvertically (ventrodorsally), while the accessory portions pass subhorizontally (caudocranially).

Origin. In Zaglossus, two first slips originate by superficial (medial-side) aponeuroses and the others have mostly fleshy origins.

In Tachyglossus, only the first slip originates by a superficial (medial-side) aponeurosis and the others have fleshy origins.

In Ornithorhynchus, all slips originate by superficial (medial-side) aponeuroses, except for the accessory portion of the last slip ( $m$. serratus ventralis cervicis VII accessorius), which origin is fleshy.

Insertion (Fig. 20). In Zaglossus, insertions of all slips together occupy at least the dorsal one-third of the internal surface of the scapula. The slip from the epistropheus inserts on the dorsal border of the scapula, except for the cranial one-tenth and the caudal one-fifth of its length. The slip from vertebra III has an even wider insertion, spreading along the entire dorsal border of the scapula and descending for one-fifth of its anterior border. The insertion of the slip from vertebra IV is widest, spreading along the entire dorsal border of the scapula and descending for more than one-third of its anterior border. The slip from vertebra V bifurcates; its accessory portion inserts as a relatively wide band on the internal side of the dorsal half of the cranial border of the scapula and a small part of its dorsal border; the main portion of the slip $\mathrm{V}$ inserts along caudal two-thirds of the dorsal border of the scapula, slightly apart from the accessory portion. In the slips from vertebrae VI and VII, the space between insertions of the main and accessory portions is much greater. Their main portions insert at the dorsocaudal angle of the scapula one above the other - the attachment of the VIIth is below that of the VIth and spreads onto the caudal border of the scapula. The respective accessory portions insert on the cranial half of the internal surface of the scapula one after another - the attachment of the VIIth is caudal to that of the VIth.

In Tachyglossus, the slip from the epistropheus inserts along almost the entire dorsal border of the scapula. The insertions of the slips from vertebrae III and IV spread not only along the entire dorsal border of the scapula, but also descend onto its cranial border (the slip from vertebra IV descends more ventrally than that from vertebra III). In the double slip from vertebra $\mathrm{V}$, the accessory portion inserts as a wide band along the dorsal half of the cranial border of the scapula, and the main portion inserts along the caudal half of its dorsal border. The accessory and main portions of the slip from vertebra VI insert, respectively, caudal and ventral to the same portions of the preceding slip. The accessory portion of the slip from vertebra VII inserts similarly, caudal to the accessory portion of the VIth slip, and the main portion of slip VII inserts along the dorsal half of the caudal border of the scapula.

In Ornithorhynchus, insertions of all slips are assembled on the internal surface of the scapula close to its dorsal and cranial borders and, on the whole, occupy a considerably smaller fraction of this surface (covered mostly by the $m$. subscapularis) than in Tachyglossidae. The slip from the epistropheus forms a wide fan inserting on the craniodorsal angle of the scapula and along its dorsal border, except for the dorsocaudal angle. Other slips are double. In the slip from vertebra III, the main portion inserts along the dorsal border of the scapula just ventral to caudal fibres of the slip from the epistropheus, and the accessory portion inserts below anterior fibres of that slip descending even lower along the cranial border of the scapula, up to its midheight. Four subsequent slips are similar in structure. The main portions insert on the internal side of the dorsocaudal angle of the scapula, each subsequent portion caudal to the preceding one, and the accessory portions insert in the same order on the lower half of the cranial part of the scapula, caudal to the $m$. levator 

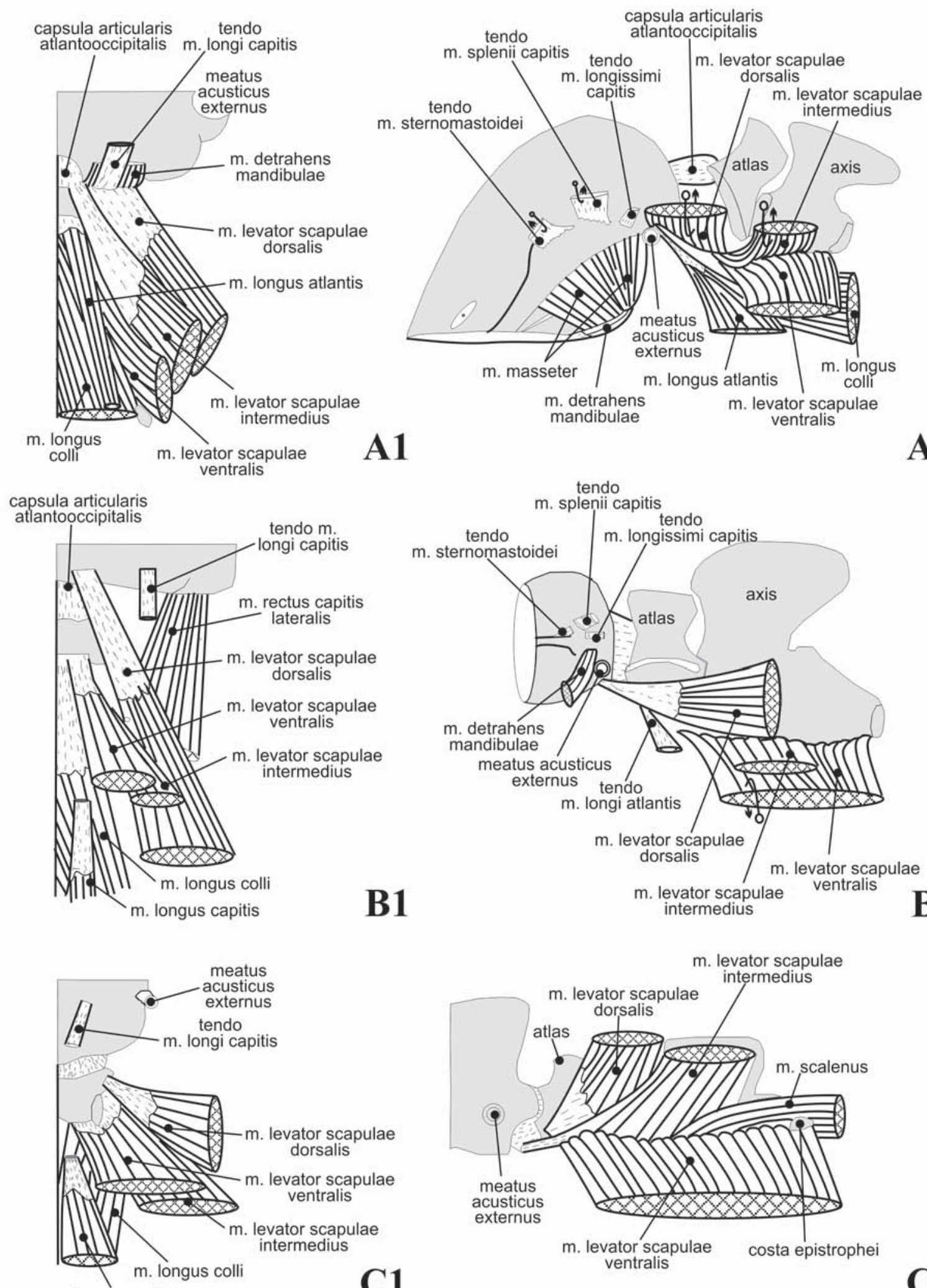

$\mathrm{m}$. longus capitis

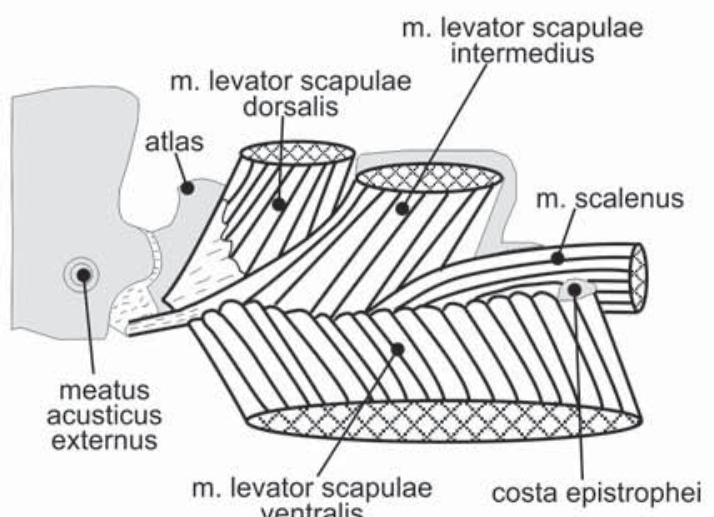

ventralis
B2

Figure 8. Details of origin of the $m$. levator scapulae. 1 - ventral view, 2 - lateral left-side view. 
scapulae intermedius and m. levator scapulae ventralis.

Remarks. The complexity of this muscle in monotremes was generally overlooked by our predecessors. McKay (1894) found its accessory portions, which he named "ventral", in Ornithorhynchus, but failed to notice them in Tachyglossus (note, that he treated the $m$. serratus ventralis cervicis as the $m$. levator scapulae, like in human anatomy). The evolutionary significance of this division was never considered, in spite of the fact that similarly bifurcating caudal slips of the homologous muscle are found in urodelans and Sphenodon (Miner, 1925). Miner called the series of subvertical (main) portions "the deeper layer of the $m$. serratus profundus", and the series of subhorizontal (accessory) portions "the superficial layer of the $m$. serratus profundus". However, the set thereof found in monotremes is apparently more complete and homonomic (especially in Tachyglossidae), and, as such, can be definitely regarded as the most primitive condition among all recent tetrapods. Under the term "accessory", for the cranial (subhorizontal) portions of the slips, we imply "additional, as compared to therians". Indeed, the $m$. serratus ventralis cervicis of therians is comparable to the $m$. serratus ventralis cervicis proprius alone of monotremes (especially, to that of Ornithorhynchus). In therians, this muscle does not insert, in the manner of accessory slip portions, on the cranial border of the scapula, each next slip below the previous one. So, the $m$. serratus ventralis cervicis accessorius was, apparently, lost in therians.

Musculus serratus ventralis thoracis $(=m$. serratus anterior superficialis) (Figs. 5, 7, 9).

Origin. Zaglossus has four slips, originating from the lower halves of four first ribs short of reaching costal cartilages; on more posterior ribs, the distance to cartilages is greater than on anterior ribs. The origins on all the ribs are approximately equal in extent. Two first slips originate from the posterior border of respective ribs; in the third and fourth, the origin also spreads onto the external rib surface.

Tachyglossus has slips from four or five first ribs, the posterior slips being longer than anterior ones. The origin of the most anterior slip occupies the entire length of the first rib from its tubercle up to connection with the costal cartilage; the origins of subsequent slips are positioned on respective ribs apart from both tubercle and cartilage. On the first rib, the origin occupies the caudal half of its external surface (posterior to attachment of the m. scalenus) and, on subsequent ribs, the origin descends over the external rib surface obliquely, from its anterior to posterior border.

Ornithorhynchus has three slips, originating from the lower halves of three first ribs short of costal cartilages. The first slip originates by a superficial, medialside aponeurosis; the second has a shorter aponeurosis of the same kind, and the origin of the third is fleshy.

Insertion (Fig. 20). In Zaglossus, the four slips fuse before reaching the scapula and insert together on the external side of the dorsocaudal angle of the scapula somewhat cranial to the scapular origin of the $m$. latissimus spinalis.

In Tachyglossus, the slips from the first and second ribs fuse close to insertion. All slips insert on the dorsocaudal angle of the scapula, not only on external, but on internal side as well.

In Ornithorhynchus, insertion is compact, located on the external side of the dorsocaudal angle of the scapula; in the first slip, it is completely fleshy, in the second, tendinous, and in the third, with a superficial, lateral-side aponeurosis, which fuses with the tendon of the preceding slip. Close to insertion, the muscle fuses with the main portion of the last slip of the $m$. serratus ventralis cervicis.

Remarks. In contrast to therians, the $m$. serratus ventralis thoracis of monotremes is underdeveloped as compared to the $m$. serratus ventralis cervicis. This may be due to the general anteroposterior inclination of the scapula: in monotremes its dorsal border is found in the cervical region, while in therians it is in the thorax, and, hence, the major weight is transmitted onto the scapula, respectively, through the cervical or through the thoracic part of the m. serratus ventralis.

Musculus omohyoideus (Figs. 6, 7, 9).

Origin. In all monotremes, this muscle originates from the hyoid cartilage.

Insertion (Fig. 20). In Zaglossus, the muscle inserts by a thin aponeurosis on the medial-side superficial fascia of the m. supraspinatus.

In Tachyglossus, it inserts by an aponeurosis in the centre of the internal surface of the scapula above the dorsal margin of the origin of the $m$. supraspinatus and below the insertion of the $m$. serratus ventralis cervicis accessorius.

In Ornithorhynchus, the muscle inserts fleshy on the internal side of the acromion, just caudal to the attachment of the clavicle and ventral to the origin of the $m$. subscapularis.

Musculus sternocoracoideus (Figs. 10, 11).

Origin (Fig. 19). In Zaglossus, the muscle originates by a short aponeurosis from the dorsal side of the sternal manubrium along the rim of the sternocoracoid joint and from the internal side of the base (sternal end) of the first costal cartilage, adjoining from below the $m$. costocoracoideus.

In Tachyglossus, it originates fleshy from the dorsal side of the sternal manubrium along the rim of the sternocoracoid joint and from the internal side of the ventral (sternal) part of a calcified region of the first costal cartilage, adjoining from below the $m$. costocoracoideus.

In Ornithorhynchus, it originates fleshy from the dorsal side of the sternal manubrium along the rim of the sternocoracoid joint, from its articular capsule, and from the internal side of the ventral (sternal) segment of the first costal cartilage, adjoining from below the $m$. costocoracoideus. 

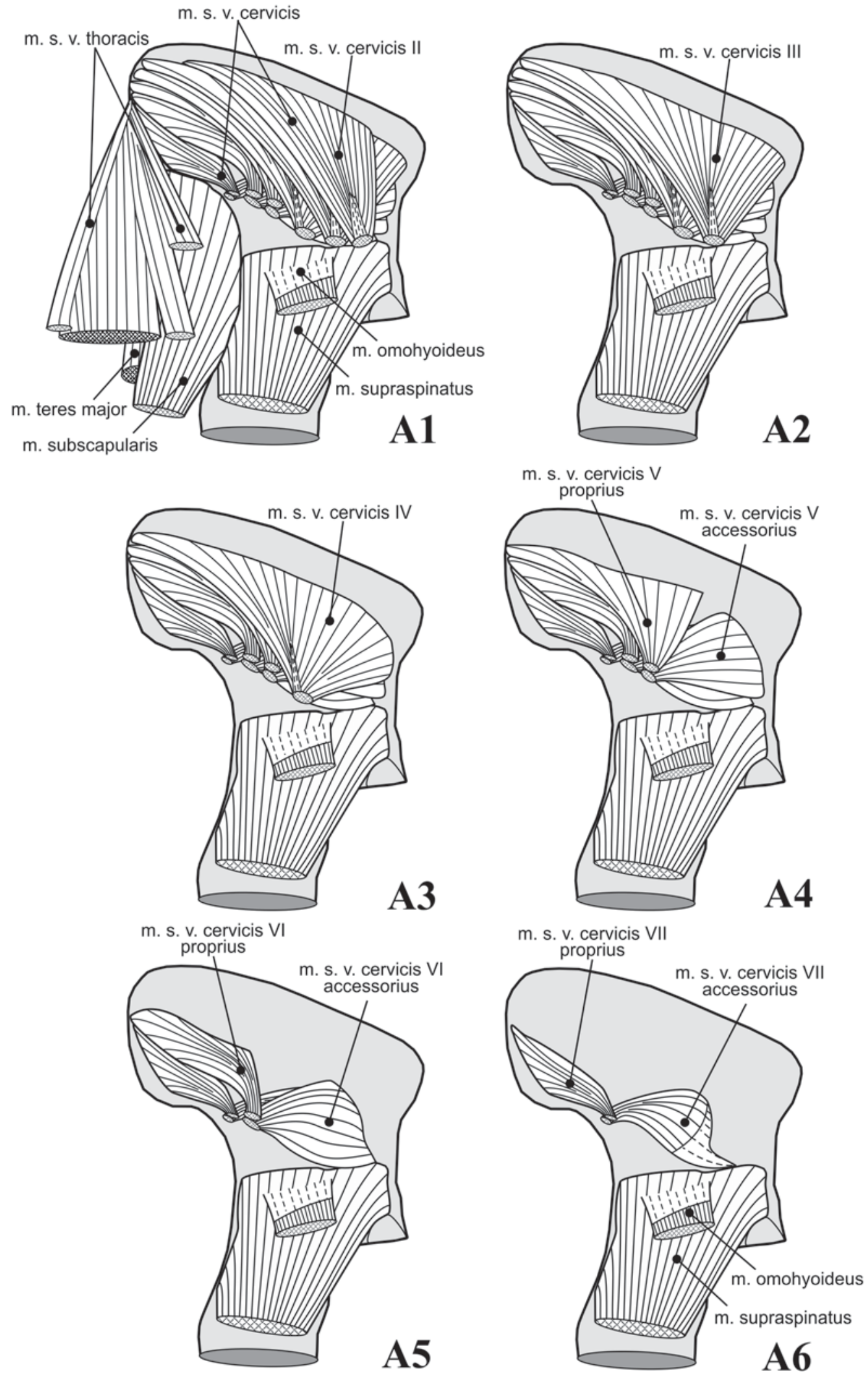

Figure 9. Details of structure of the m. serratus ventralis cervicis. Medial view. 1- all six slips, 2 - slip from vertebra II removed, 3 - slip III removed, 4 - slip IV removed, 5 - slip V removed, 6 - only slip VII retained. 

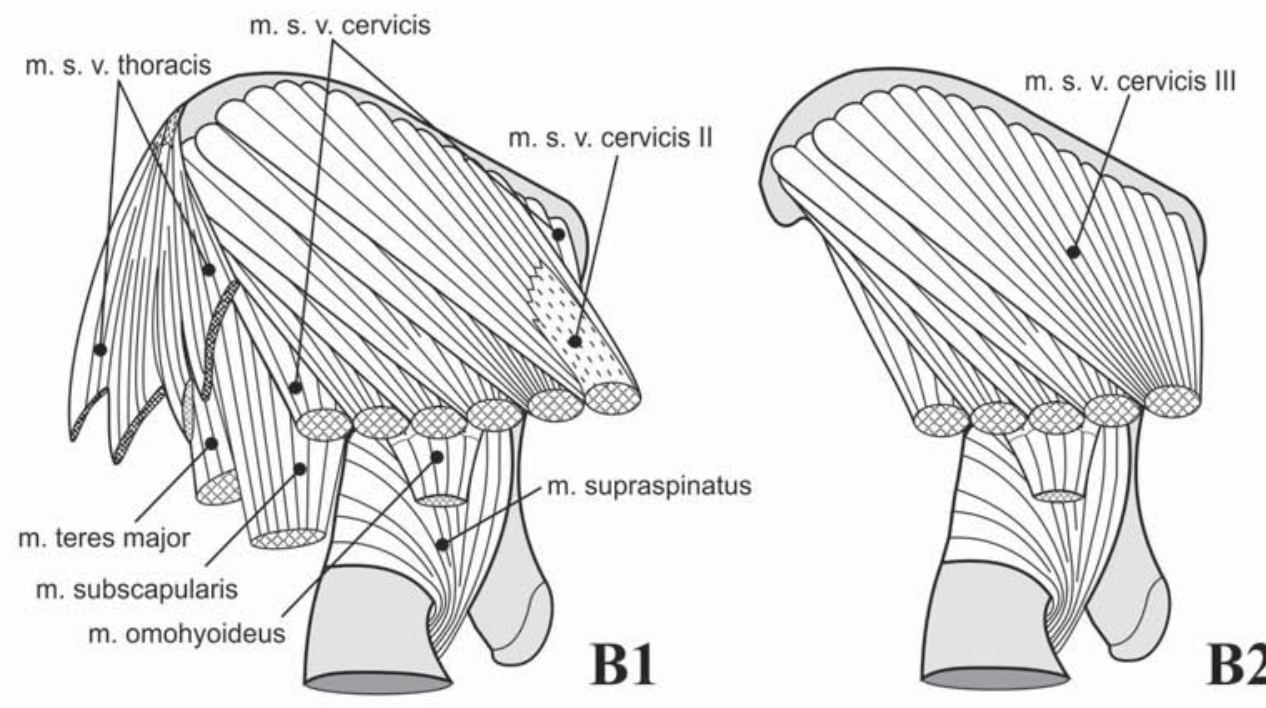

B2
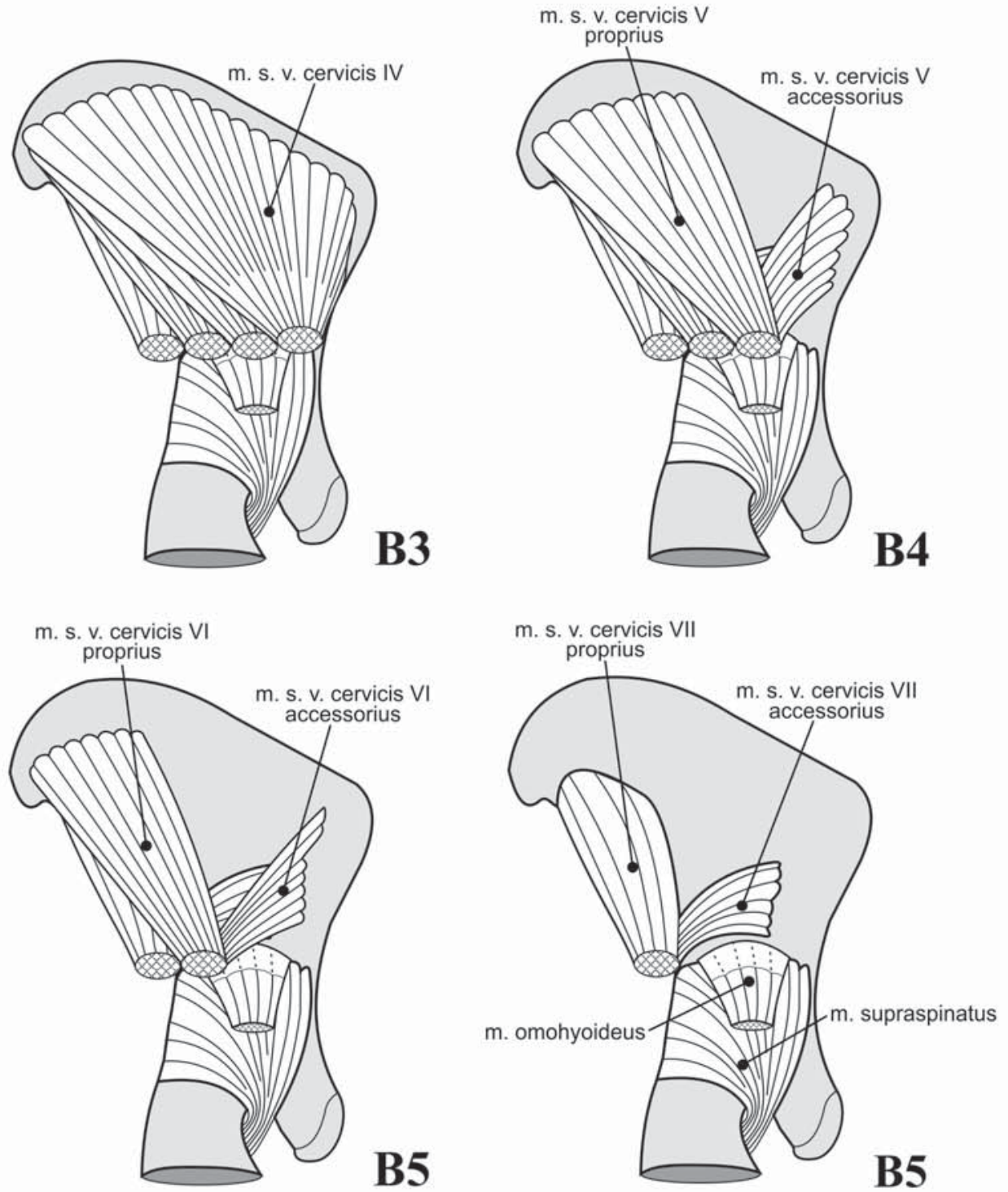

Figure 9 (continued). 

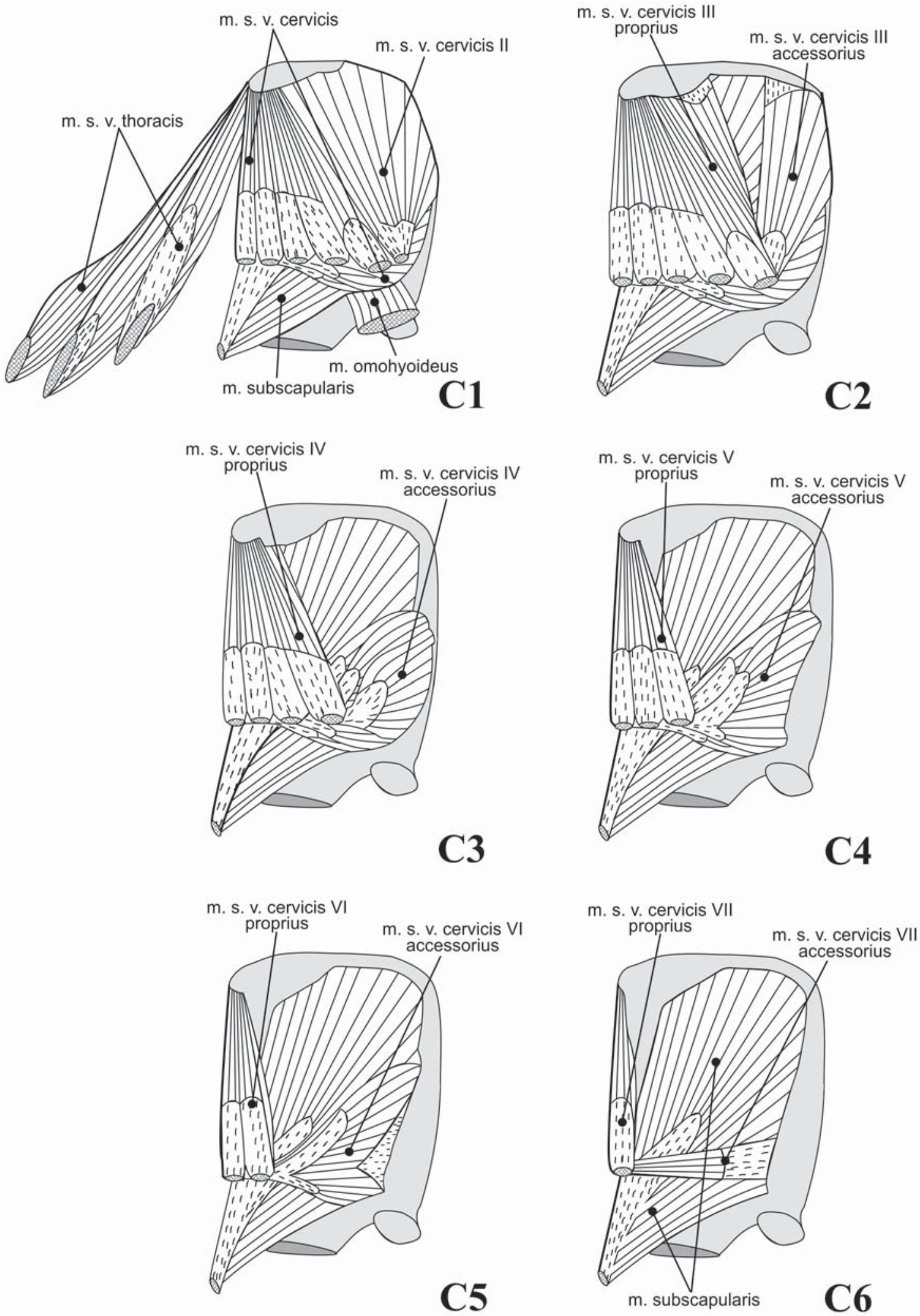

Figure 9 (continued). 


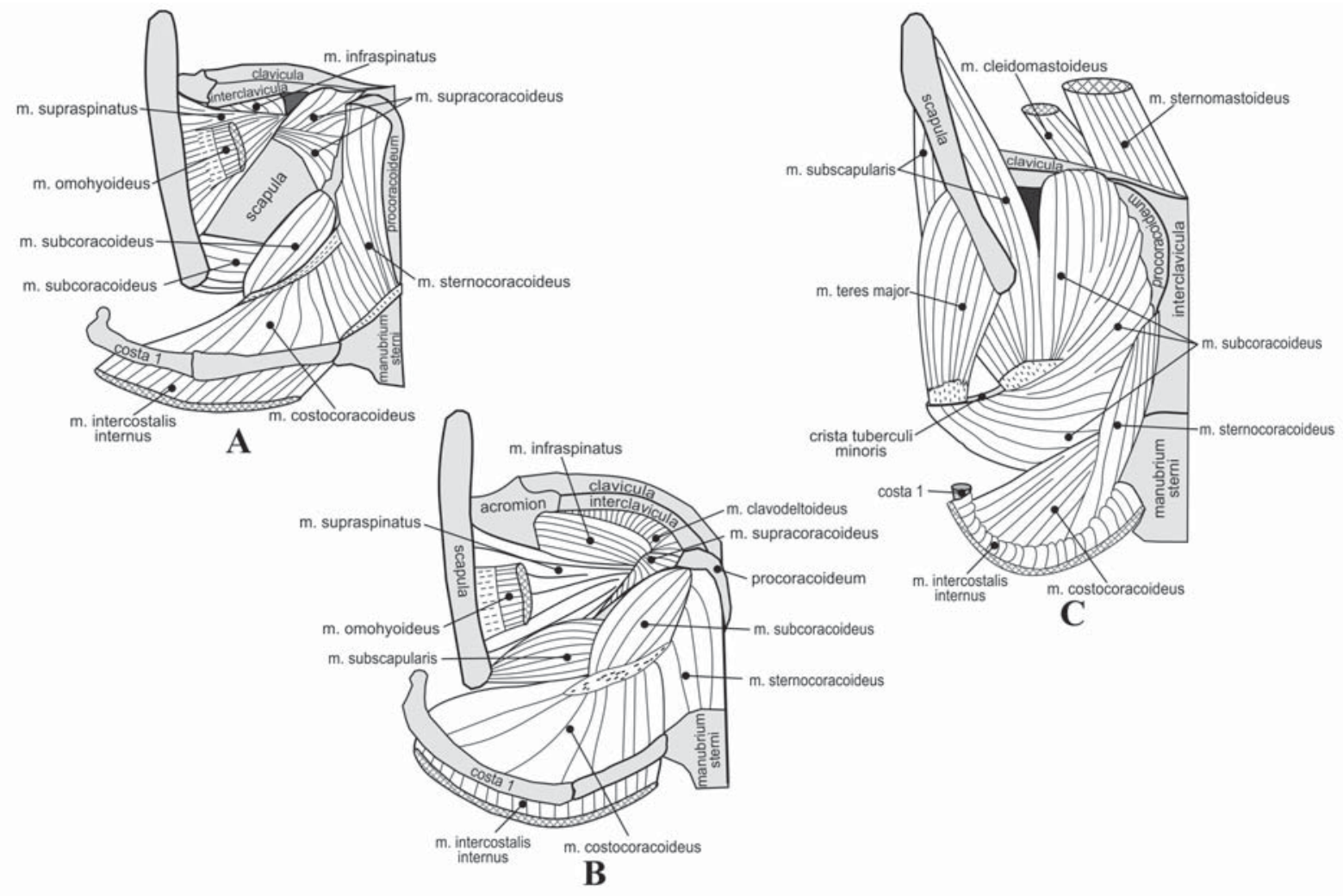

Figure 10. Inner muscles of the coracoid plate. Dorsal view.

Insertion (Fig. 19). In Tachyglossidae, the muscle inserts on the dorsal (internal) side of the medial border of the procoracoid.

In Ornithorhynchus, the muscle is unipennate; the terminal aponeurosis arises on the upper part of the lateral surface of the belly and inserts on the dorsal side of the medial border of the procoracoid in its posterior half, apart from the suture with the coracoid. The most anterior fibres insert fleshy medial to the aponeurosis on the cartilaginous lining of the procoracoid border.

Remarks. The sternocoracoid mobility is much reduced in monotremes, but the existence of the $m$. sternocoracoideus, composed of long-enough fibres, proves that it is still actual. Probably, it was even greater in basal mammals. The remnant of that condition is the specific overlapping position of the left and right procoracoids in monotremes (Fig. 1), similar to what is observed in urodelans and lizards characterized by very pronounced craniocaudal sternocoracoid mobility.

Musculus costocoracoideus (Figs. 10, 11).

Origin (Fig. 19). In Zaglossus, the muscle originates by a wide aponeurosis from the internal side of the first costal cartilage and the cranial border of the first rib itself.

In Tachyglossus, the muscle originates fleshy from the cranial border of cartilaginous and bony segments of the first rib.
In Ornithorhynchus, the muscle originates fleshy from the cranial border of the upper part of the first costal cartilage and the lower part of the first rib itself, ascending slightly above the origin of the anterior slip of the $m$. serratus ventralis thoracis (between the two muscles, the $m$. scalenus is wedging).

Insertion (Fig. 19). In Zaglossus, the muscle inserts by a short aponeurosis on the dorsal (internal) side of the medial border of the coracoid and, also, on the procoracoid, just lateral to the posterior part of the $m$. sternocoracoideus.

In Tachyglossus, it inserts by a short aponeurosis on the dorsal side of the medial border of the coracoid adjoining the $m$. sternocoracoideus at the procoracoidcoracoid suture.

In Ornithorhynchus, the insertion is restricted to the posterior half of the dorsal side of the medial border of the coracoid and, so, does not reach anteriorly the suture with the procoracoid.

\section{Dorsal muscles from trunk to limb}

Musculus latissimus spinalis (Figs. 5, 6, 11, 13).

Origin (Fig. 20). In Zaglossus, the muscle originates from the neural spines of thoracic vertebrae, beginning from 1 st or 2 nd to 11 th or 12 th. Up to the 5 th or 6 th vertebra, the origin is fleshy and, then, it is aponeurotic. This aponeurosis becomes gradually longer 


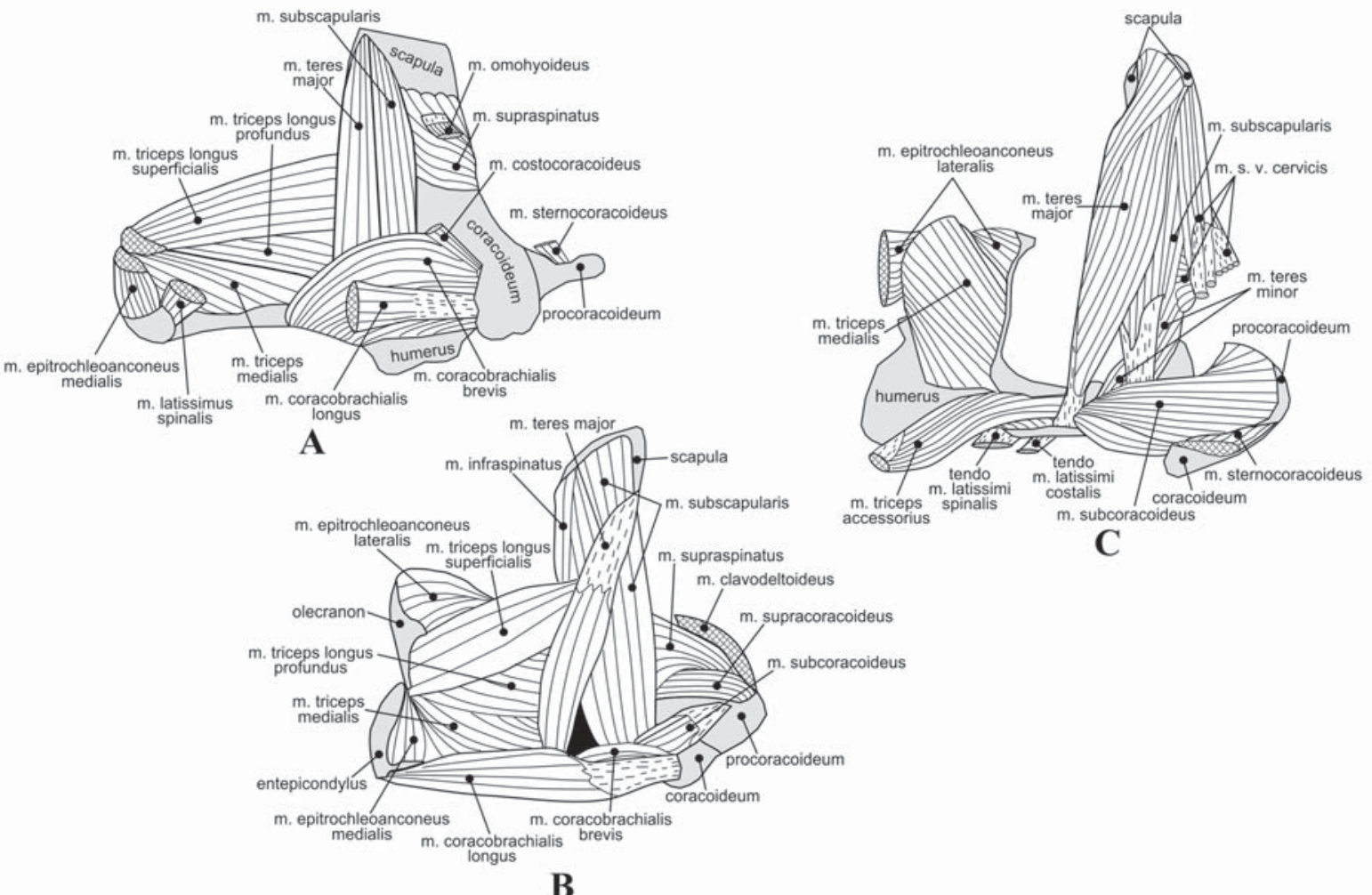

Figure 11. Shoulder muscles. Posterior view.

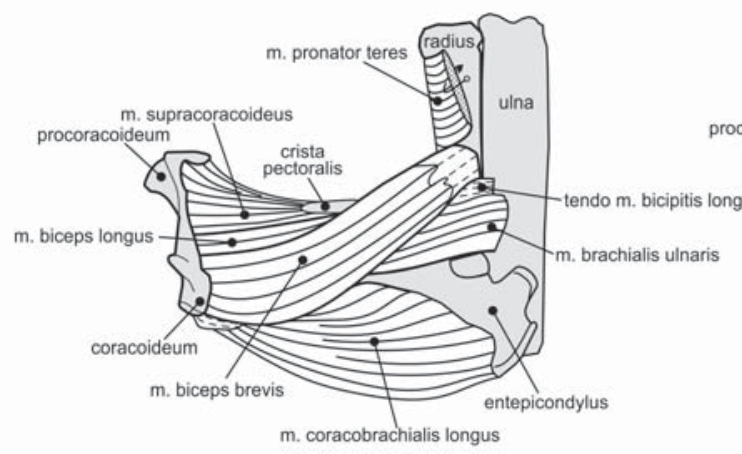

B1

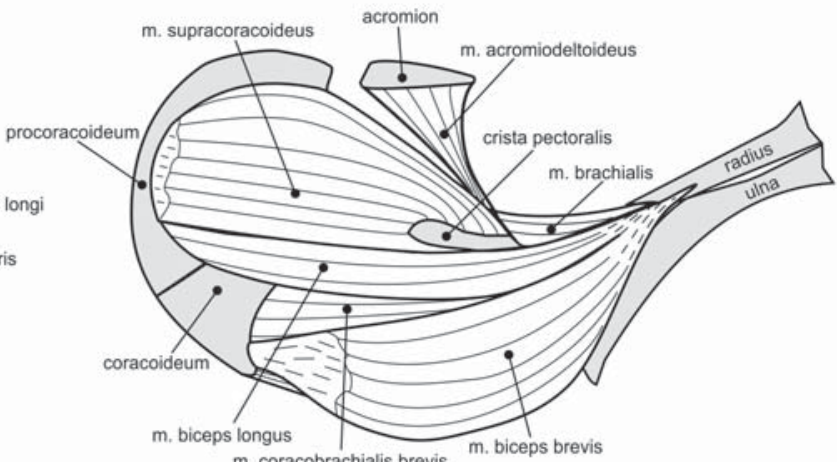

$$
\text { m. coracobrachialis brevis }
$$

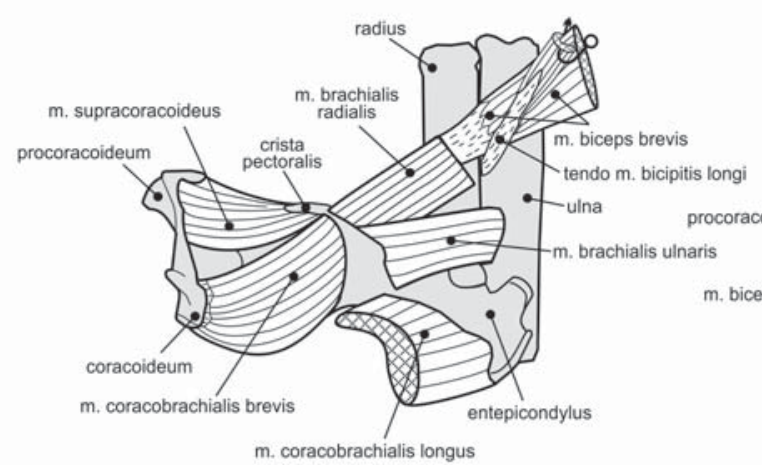

B2
C1

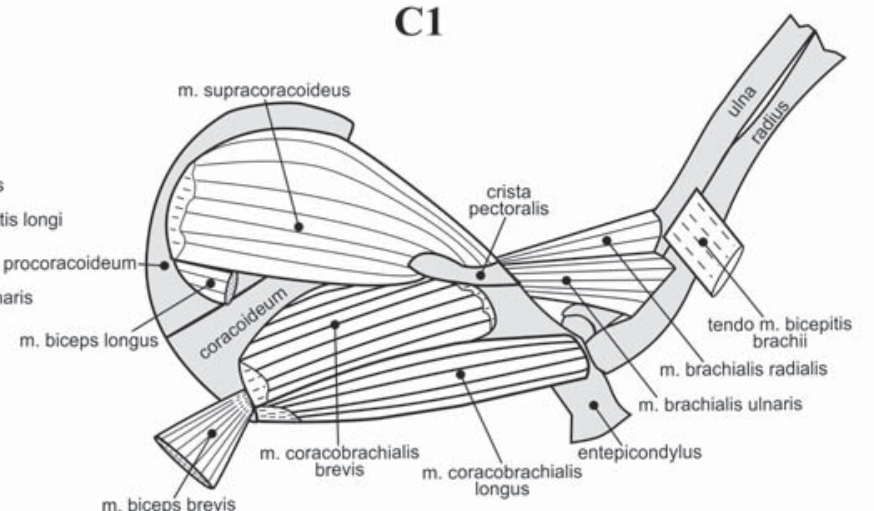

$\mathrm{C2}$

Figure 12. Ventral muscles of the humerus of Tachyglossus and Ornithorhynchus. Ventral view. 1 - superficial layer (the $m$. pectoralis and $m$. clavodeltoideus are removed), 2 - deep layer. 

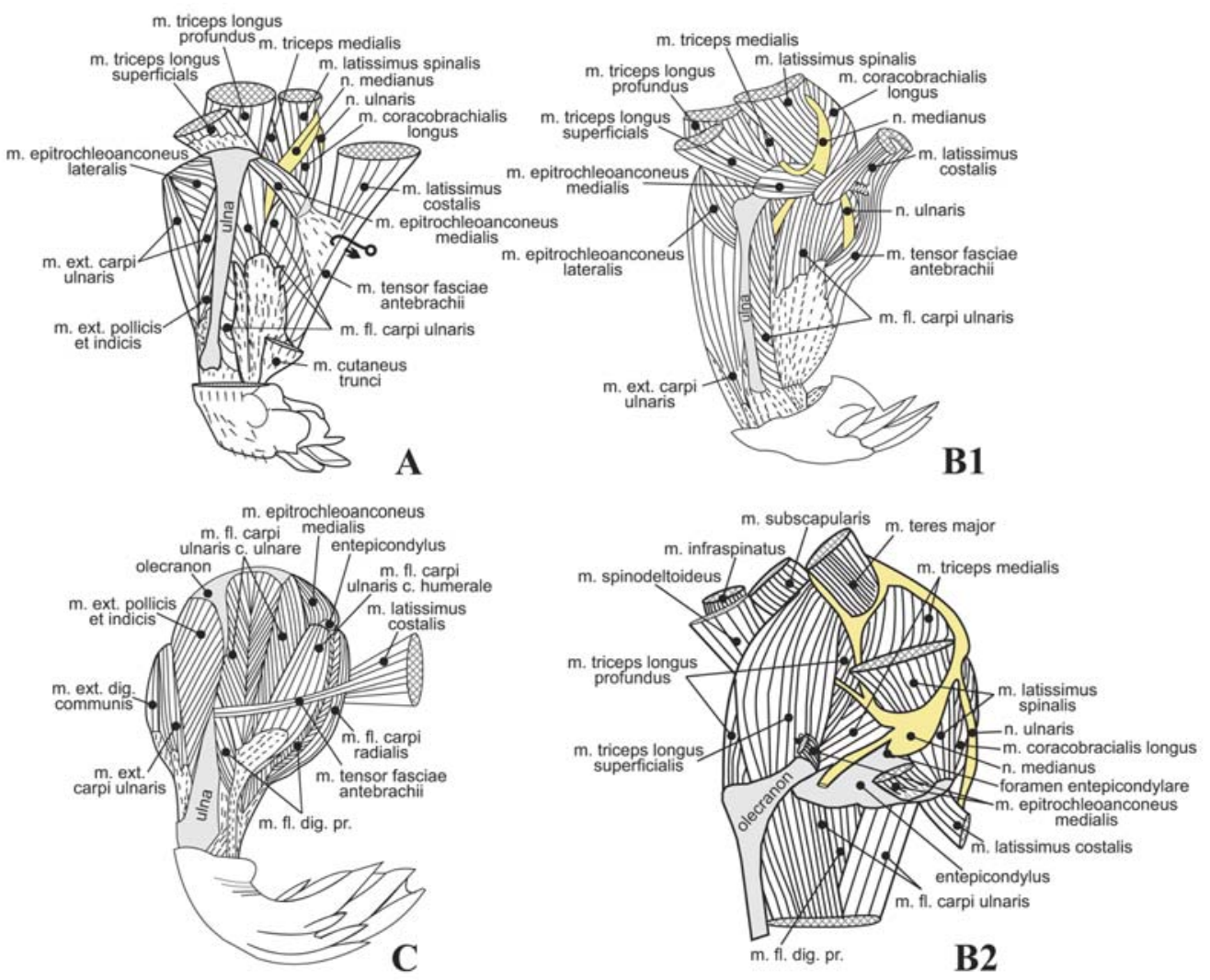

Figure 13. Forearm muscles. Lateral view. 2 - details of the nerve routes at the elbow (shown only for Tachyglossus).

from the 6th to 11 th vertebrae. In addition, there are slips originating fleshy from ribs 7 and 8 (in one specimen, only from the 7 th) slightly above the middle of the rib, over approximately one-sixth of its length. Also, there is a slip originating from the external surface of the ventral part of the dorsocaudal angle of the scapula.

In Tachyglossus, the muscle originates from the neural spines of thoracic vertebrae 2-10 (or 11); up to vertebra 7 , the origin is fleshy and, then, it is aponeurotic, with gradually elongating aponeurosis. In addition, it originates from the external surface of the ventral part of the dorsocaudal angle of the scapula and from the proximal (upper) one-third of the aponeurosis of origin of the $m$. teres major.

In Ornithorhynchus, it originates from the neural spines of thoracic vertebrae 3-11; up to vertebra 7, the origin is fleshy and, then, it is by gradually elongating aponeurosis.

Insertion (Fig. 21). In Tachyglossidae, the muscle inserts by an aponeurosis on the special tubercle (Fig. 2) on the dorsal side of the posterior border of the entepicondylus of the humerus (for Tachyglossus, Walter (1988) described it as insertion on the "internal condyle of humerus" and did not come into further detail). The terminal part of the muscle is embraced by nerve branches converging to the entepicondylar foramen and, thus, separating this muscle from the $m$. epitrochleoanconeus medialis and the m. coracobrachialis longus (Fig. 13).
In Ornithorhynchus, the insertion is mainly fleshy, but is aided by an aponeurosis on the external (ventrolateral) surface of the terminal part of the muscular belly. The insertion descends along the postaxial aspect of the humerus from the distal angle of the pectoral crest (closely adjoining here the $m$. latissimus costalis) to the entepicondylar foramen forming a distinct facet on the bone.

Remarks. In respect of the origin on the spine, the $m$. latissimus spinalis of monotremes is comparable to the whole $m$. latissimus dorsi of therians. However, in the latter, the insertion is at the lesser tubercle of the humerus, near the insertion of the $m$. teres major, while, in monotremes, it is far away on the opposite end of the humerus, at the entepicondylus. In recent reptiles, the insertion is like in therians, so the condition found in monotremes should be regarded as secondary. Apparently, it was acquired together with peculiar barrel-like shape of their rib cage. With this body build, the elbow falls in the same parasagittal plane as the lateral-most points of the ribs and, hence, the $m$. latissimus dorsi descends in this plane along the sides of the rib cage directly onto the entepicondylus.

Musculus latissimus costalis (Figs. 5, 6, 11, 13).

Origin. In Zaglossus, the muscle originates as slips from five, six, or seven ribs, beginning from rib 8 , above the boundary with their costal cartilages. Caudally, the origins of slips are gradually placed lower, i.e., 
closer to the costal cartilages. In particular, on rib 8, the slip originates from the middle of its length, while in rib 13 , just near the costal cartilage. The extent of origin on the rib increases posteriorly.

In Tachyglossus, it originates from ribs 8-14.

In Ornithorhynchus, it originates from ribs 8-15.

Insertion (Fig. 21). In Zaglossus, according to Allen (1912), the insertion is on "the most posterior part of the tuberosity of the humerus" but, according to our dissection, the muscle inserts by two aponeuroses. The first one arises from the anterior side of the muscular belly and inserts on the dorsal side of the posterior border of the entepicondylus, somewhat more distally than the $m$. latissimus spinalis. There is a narrowing of the muscular belly at this point, distal to which the muscular fibres continue and pass into the second aponeurosis, which is in fact the fascia antebrachii.

In Tachyglossus, the insertion is also double. The anterior muscular fibres insert via aponeurosis on the dorsal side of the posterior border of the entepicondylus just distal to the origin of the $m$. epitrochleoanconeus medialis. The posterior muscular fibres of the $m$. latissimus costalis bypass the entepicondylus and connect to the $m$. tensor fasciae antebrachii so that the most superficial fibres of the first muscle pass into those of the second one directly, while the deeper fibres do so via a tendinous interseptum. Thus, the two muscles make up a kind of digastric complex with the proximal belly represented by the $m$. latissimus costalis, the distal belly represented by the $m$. tensor fasciae antebrachii, and a definite narrowing at the interseptum between the two (Fig. 13B). However, in one specimen of Tachyglossus, the muscle looked like in Zaglossus: there was no interseptum, and even the connection with the entepicondylus was absent, so that the $m$. latissimus costalis passed into the $m$. tensor fasciae antebrachii directly.

In Ornithorhynchus, the insertion of the muscle is sharply different. Its terminal aponeurosis arises on the lower edge of the muscular belly and passes into a flat tendon, which inserts not distal to the $m$. latissimus spinalis but just proximal to it, along the posterior (morphologically ventral) side of the pectoral crest of the humerus.

Remarks. Judging from the origin on the ribs and taking into account the presence of the $m$. latissimus spinalis, the monotreme-like $m$. latissimus costalis is lacking beyond this group, which shows two sharply different variants of humeral insertion of this muscle. In Tachyglossidae, the insertion is found distal to that of the m. latissimus spinalis, while, in Ornithorhynchus, the order is reversed.

Musculus tensor fasciae antebrachii $(=m$. dorsoepitrochlearis) (Figs. 6, 13, 16).

Origin. In Zaglossus, this muscle is the direct continuation of the $m$. latissimus costalis. The narrowing of the muscular belly of the latter near the entepicondylus may be regarded as an arbitrary beginning of the $m$. tensor fasciae antebrachii.
In Tachyglossus, this muscle is the continuation of posterior fibres of the $m$. latissimus costalis; the transition from one to another is marked not only by the narrowing of the muscular belly, but also by a tendinous interseptum. As was noted above, in one specimen of Tachyglossus, the muscle looked exactly like in $\mathrm{Za}$ glossus: there was no interseptum, so that the $m$. tensor fasciae antebrachii was a direct continuation of the $m$. latissimus costalis.

In Ornithorhynchus, this relatively small muscle does not continue directly the $m$. latissimus costalis but arises from the superficial fascia of its belly just proximal to the beginning of its terminal tendon (Fig. 6C3).

Insertion (Fig. 22C). In Tachyglossidae, the fibres of this muscle insert on the fascia antebrachii which, laterally, fuses with the terminal aponeurosis of the $m$. flexor carpi ulnaris and, distally, passes into the fascia palmaris manus.

In Ornithorhynchus, the muscle inserts on the lateral (postaxial) crest of the ulna near its midlength.

Remarks. In Ornithorhynchus, this muscle deviates from the $m$. latissimus costalis like, in therians, it deviates from the $m$. latissimus dorsi $(=m$. latissimus spinalis). Among Tachyglossidae, Zaglossus and one specimen of Tachyglossus lacked any tendinous interseptum between the $m$. latissimus costalis and the $m$. tensor fasciae antebrachii. However, in the same place, near the elbow, Zaglossus has a narrowing of the muscular belly, and, hence, it is possible to regard the antebrachial segment of the $m$. latissimus costalis, which is positioned distal to this narrowing, as a homologue of the $m$. tensor fasciae antebrachii. Probably, the unity of the two muscles found in Zaglossus and one specimen of Tachyglossus is primitive. If so, the tendinous interseptum found in the other specimen of Tachyglossus represents an intermediate condition between $\mathrm{Za}$ glossus and Ornithorhynchus, and, in therians, the $m$. tensor fasciae antebrachii is the last remnant of the $m$. latissimus costalis.

\section{Ventral muscles from trunk to limb}

Musculus pectoralis (Figs. 6, 7) is not divided in monotremes into the $m$. pectoralis superficialis and the $m$. pectoralis profundus as is typical to therians.

Origin (Fig. 19). In Zaglossus, the muscle originates along anterior one-fifth of the stem of the interclavicle (except for its most cranial part).

In Tachyglossus, it originates along anterior onefourth of the stem of the interclavicle (except for its most cranial part) and, further posteriorly, from the sternum excluding the xiphoid process.

In Ornithorhynchus, it originates along anterior onefifth of the stem of the interclavicle (except for its most cranial part) and from the ventral (sternal) parts of costal cartilages of the first five ribs.

Insertion (Fig. 21). In Zaglossus, the insertion is on the ventral side of the pectoral crest of the humerus and covers about $10 \%$ of the bone length. 


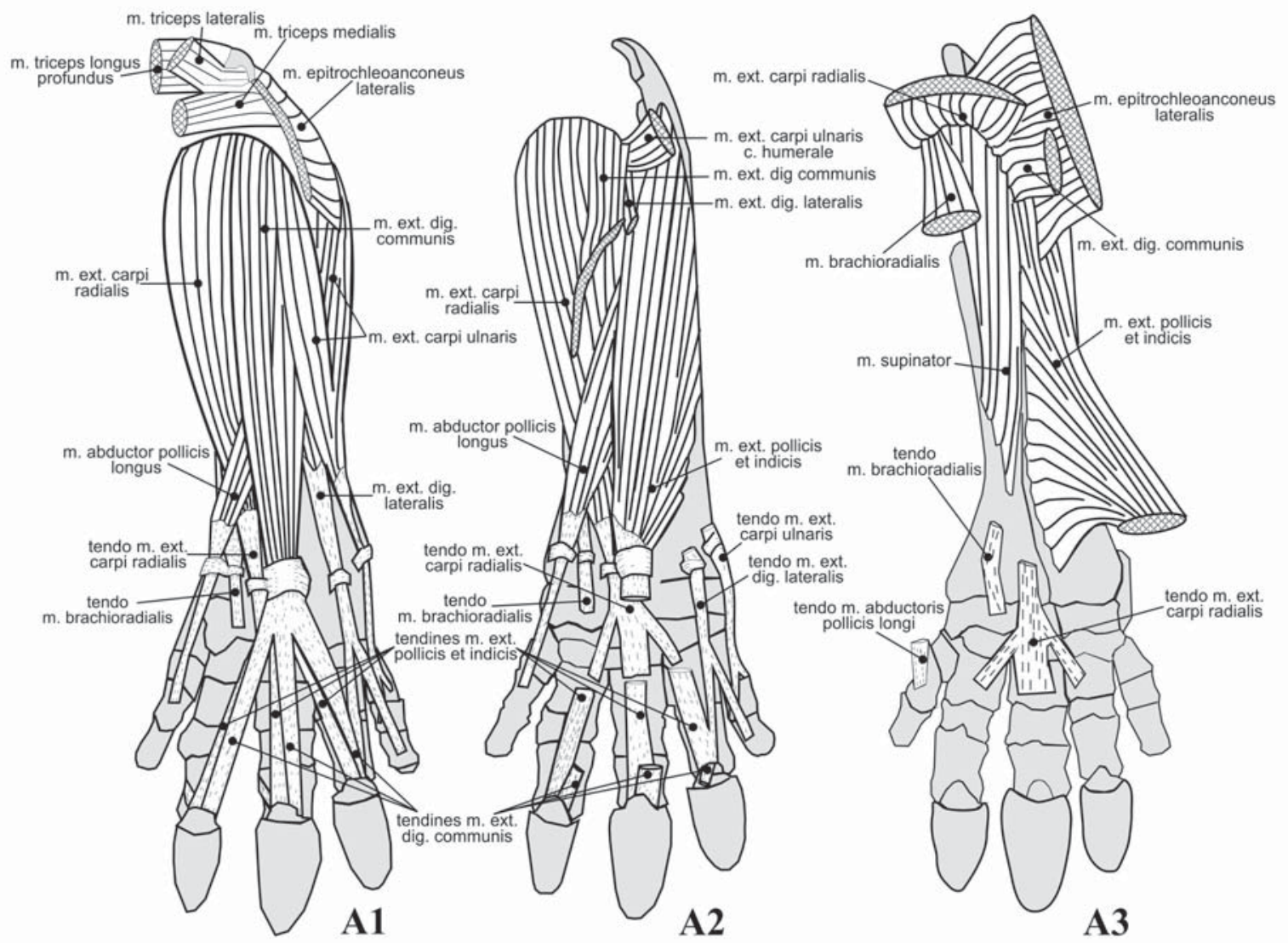

Figure 14. Forearm extensors.

In Tachyglossus, the muscle inserts on the compact area of the ventral side of the pectoral crest of the humerus.

In Ornithorhynchus, it inserts along the whole length of the pectoral crest, on its ventral side.

Musculus pectoralis abdominalis (Figs. 6, 7).

Origin (Fig. 19). In Zaglossus, the muscle originates from costal cartilages of ribs $6-8$ somewhat apart from the midline of the abdomen.

In Tachyglossus, the muscle originates from the cranial one-third of the midline of the abdomen.

In Ornithorhynchus, it originates from the cranial one-fourth of the midline of the abdomen.

Insertion (Fig. 21). In Zaglossus, the muscle inserts on the pectoral crest of the humerus just distal to the $m$. pectoralis.

In Tachyglossus, it inserts on the pectoral crest over a greater extent than in Zaglossus, displacing proximally the insertion of the $m$. pectoralis.

In Ornithorhynchus, only some fibres of this muscle insert, as in Tachyglossidae, on the pectoral crest just distal to the insertion of the $m$. pectoralis via a separate tendon, but most fibres terminate just on the posterior edge of the latter muscle.
Musculus cutaneus trunci $(=m$. panniculus carnosus) (Figs. 6, 13, 14, 16, 18).

Origin. In all monotremes, this muscle spreads subcutaneously over the whole body, being anchored, anteriorly, to the parietale and the chin and, posteriorly, to the transverse processes of the tail vertebrae, cloacal orifice, and the hindlimb. The division of the muscle into portions and their associations beyond the forelimb are the subject of the other paper.

Insertion (Figs. 21, 22). In all monotremes, many fibres of this muscle converge from the body flank at the axilla to form the main insertion on the pectoral crest, wrapping the endings of the $m$. pectoralis and $m$. pectoralis abdominalis from the dorsal, distal, and ventral sides, and the additional insertion, via the fascia antebrachii, on the postaxial border of the ulna. In Tachyglossidae, the second insertion is at the distal end of the ulna, while in Ornithorhynchus, it is close to its midlength. Also, a bundle of muscular fibres fuses into a fascia coating the extensor (anterior) surface of the antebrachium; this connection is most pronounced in Ornithorhynchus (see below).

In Zaglossus, a bundle of fibres of this muscle descends along the flexor (posterior) side of the antebrachium to the wrist joint and, via the fascia palmaris manus, connects to the preaxial side of the common 

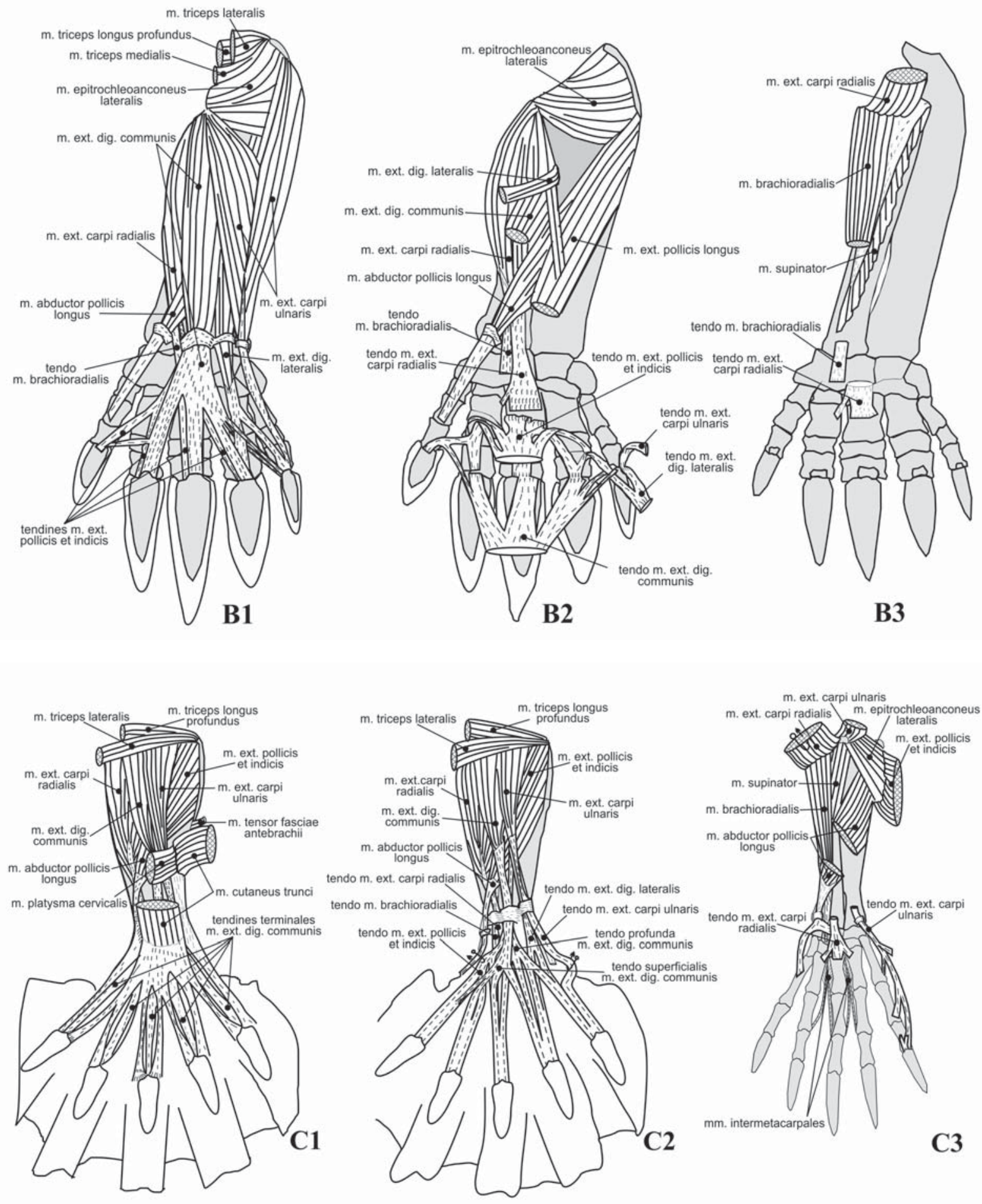

Figure 14 (continued).

tendon of the $m$. flexor digitorum profundus near its branching point (Fig. 18A).

In Ornithorhynchus, the muscle spreads much onto the extensor side of the antebrachium and, at the wrist joint, terminates by subcutaneous aponeurosis, which, then, splits into four interdigital branches. Finally, each branch bifurcates to insert on opposing sides of the claws of adjacent digits (Fig. 14C).

Remarks. A muscular bundle, found in Zaglossus, which is associated with the fascia palmaris manus and the common tendon of the m. flexor digitorum profun$d u s$, is the apparent predecessor of the therian m. palmaris longus. 


\section{Dorsal muscles of shoulder joint}

Musculus clavodeltoideus (Fig. 7).

Origin (Fig. 19). In Zaglossus, the origin of this muscle covers the caudal border of the clavicle and the lateral ramus of the interclavicle, through the distance from the level of the procoracoid to acromion of the scapula.

In Tachyglossus, the muscle originates from the entire ventral surface of the clavicle, except for its acromial end and the entire ventral surface of the lateral ramus of the interclavicle. In the medial part, this muscle has a superficial aponeurosis expanding on its ventral side for almost one-fourth of its length.

In Ornithorhynchus, this muscle is inseparable from the $m$. acromiodeltoideus and they embrace together the $m$. spinodeltoideus from below: the fibres located caudal to the last can be regarded as the m. acromiodeltoideus and the fibres located cranially, as the $m$. clavodeltoideus. So, it originates from the ventral surface of the acromion (completely fusing here with the underlying $m$. acromiodeltoideus), ventral surface of the lateral ramus of the interclavicle, and the clavicle spreading medially up to the insertion of the $m$. sternomastoideus.

Insertion (Fig. 21). In Zaglossus, the muscle inserts widely on the anterior part of the proximal dorsal surface of the humerus including its pectoral crest.

In Tachyglossus, it inserts on the dorsal side of the pectoral crest of the humerus. The most distal fibres insert via a short strong tendon.

In Ornithorhynchus, its insertion descends along the pectoral crest and far distal to it.

Musculus spinodeltoideus $(=m$. dorsalis scapulae $=m$. deltoideus scapularis of Diogo \& Abdala, 2010) (Figs. 5, 6).

Origin (Fig. 20). In all monotremes, this muscle originates from the edge of the craniodorsal angle of the scapula.

In Ornithorhynchus, the origin of this muscle is split by the insertion of the $m$. spinotrapezius so that the cranial muscular fibres arise from the scapula over the latter muscle while the caudal fibres comes out to the surface from under it.

Insertion (Fig. 21). In Zaglossus, at mid-height of the scapula, the muscular belly passes into a tendon, which inserts on the proximal dorsal surface of the humerus between the $m$. clavodeltoideus and $m$. acromiodeltoideus, posterior to the former and anterior to the latter.

In Tachyglossus, the muscle also inserts between the $m$. clavodeltoideus and $m$. acromiodeltoideus on the proximal dorsal surface of the humerus, but closer to the pectoral crest than in Zaglossus.

In Ornithorhynchus, muscular fibres attach to aponeuroses on the lateral and posterior sides of the belly which pass into a terminal tendon inserting on the dorsal side of the pectoral crest between the m. clavodeltoideus and m. acromiodeltoideus.
Musculus acromiodeltoideus (Figs. 7, 12).

Origin (Figs. 19, 20). In Tachyglossidae, this muscle only originates from the apex of the acromion.

In Ornithorhynchus, this muscle is not listed by Diogo \& Abdala (2010); it originates from the posterior side of the acromion, slightly expanding onto the adjacent part of the clavicle.

Insertion (Fig. 21). In Zaglossus, the muscle inserts on the proximal dorsal surface of the humerus by an aponeurosis which embraces from posterior the $\mathrm{m}$. spinodeltoideus and fuses, proximal and distal to the latter, with the $m$. clavodeltoideus.

In Tachyglossus, it inserts on the proximal dorsal surface of the humerus posterior to the $m$. clavodeltoideus and $m$. spinodeltoideus.

In Ornithorhynchus, it inserts along the dorsal side of the pectoral crest of the humerus proximal and posterior to the terminal tendon of the $m$. spinodeltoideus.

Remarks. In reptiles, there are only two deltoid muscles, which correspond to the mammalian $m$. clavodeltoideus and $m$. spinodeltoideus $(=m$. dorsalis scapulae). The mammalian m. acromiodeltoideus may be a portion of one of these two. However, its partially submerged position and wide insertion on the humerus in monotremes suggest, that it is a portion of the reptilian $m$. scapulohumeralis anterior, whose deeper portion is the mammalian $m$. teres minor.

Musculus teres minor (=m. scapulohumeralis anterior of Diogo \& Abdala, 2010) (Fig. 11).

Origin (Fig. 20). In Tachyglossidae, this muscle originates from a notch in the cranial border of the scapula between the acromion and the shoulder joint and, partially, from the joint capsule.

In Ornithorhynchus, it originates fleshy and by a lateral surface aponeurosis from the external side of the scapula just anterior to the $m$. triceps longus profundus. On the bone surface, the origin looks like a fossa, where fibres arise, and a ridge along the anterior border of the fossa, from which the aponeurosis originates. In addition, the muscle partially originates from the articular capsule of the shoulder joint.

Insertion (Fig. 21). In all monotremes, the muscle inserts fleshy in the fossa on the dorsal side of the lesser tubercle of the humerus immediately anterior to the insertion of the $m$. subscapularis.

Remarks. In contrast to therians, the muscle does not make a shortcut from the scapula to the humerus to insert on its greater tubercle but, instead, passes postaxially below the $m$. triceps longus to insert on the lesser tubercle of the humerus, near the $m$. subscapularis. As compared to lizards, the muscle in question looks like a posteromedial (postaxial) subdivision of the $m$. scapulohumeralis anterior, whereas the $m$. teres minor of therians, to the anterolateral portion of the same muscular mass. Shrivastava (1962) suggested that the true homologue of the therian $m$. teres minor is fused, inseparably, into the $m$. infraspinatus of monotremes. 
Musculus teres major (Figs. 6, 9, 11, 13).

Origin (Fig. 20). In Zaglossus, the origin of this muscle occupies the external side of the apex of the dorsocaudal angle of the scapula and, slightly, extends onto its internal side below insertions of the $m$. serratus ventralis.

In Tachyglossus, the origin of this muscle occupies the external side of the apex of the dorsocaudal angle of the scapula and, also, its internal side, where it wedges between the insertions of the $m$. serratus ventralis cervicis (which is more cranial) and the $m$. serratus ventralis thoracis (which is more caudal).

In Ornithorhynchus, the origin of this muscle extends along the external side of the caudal half of the dorsal border of the scapula up to the apex of its dorsocaudal angle, where the $m$. serratus ventralis thoracis inserts.

Insertion (Fig. 21). In all monotremes, the muscle inserts on the dorsal side of the distal part of the crest of the lesser tubercle of the humerus between the insertion of the $m$. subcoracoideus and the origin of the $m$. triceps accessorius.

Musculus subscapularis (Figs. 5, 6, 9-11).

Origin (Fig. 20). In Tachyglossidae, the muscle originates from the external surface of the scapula caudal to the crest where the $m$. triceps longus arises, from the caudal border of the scapula, and a narrow zone of the internal surface along it.

In Ornithorhynchus, the muscle has two incompletely fused heads, medial and laterocaudal. The latter is approximately twice as massive as the former and originates from the caudal border of the scapula and caudal part of its external surface (except for the dorsocaudal angle, where the $m$. teres major originates); anteriorly, the area of its origin is bordered by those of the $m$. triceps longus and $m$. infraspinatus. The medial head widely covers the internal surface of the scapula wedging between the $m$. serratus ventralis cervicis proprius and the m. serratus ventralis cervicis accessorius. The origins of the two heads both descend along the scapula up to the level of the acromion.

Insertion (Fig. 21). In Tachyglossidae, the muscle is bipennate. Its anterior and posterior fibres converge from the two sides on an aponeurosis passing inside the belly and, then, fusing into a superficial terminal aponeurosis on the medial surface of the belly. The insertion is on the proximal apex of the lesser tubercle of the humerus.

In Ornithorhynchus, the muscle has two superficial terminal aponeuroses; one is on the laterocaudal side of the laterocaudal head and the other is on the medial side of the medial head; these aponeuroses come in contact distally, although the boundary between them is seen up to the very end. Between these aponeuroses, nerves enter the belly. At the insertion of this muscle, the proximal apex of the lesser tubercle of the humerus bears a sesamoid bone connected with the lesser tubercle by a synovial joint. The aponeurosis of the medial head inserts on the sesamoid and the aponeurosis of the laterocaudal head inserts directly on the lesser tubercle of the humerus just distal to the sesamoid.

Remarks. At first sight, judging by the name of the muscle and by its subscapular position in therians, as well as by similar position of its homologue in nonmammalian tetrapods, its external origin in Tachyglossidae may seem to be an advanced condition, and the half-external origin in Ornithorhynchus may seem intermediate. Note, however, that the similar half-external $m$. subscapularis sometimes occurs in primitive placentals, e.g., in the giant anteater. Also, the ancient nature of the suprascapular position of this muscle is supported by the state of the muscles which "replace" it on the internal surface of the scapula in Tachyglossidae. They are the $m$. serratus ventralis cervicis and the $m$. supraspinatus. Definitely, the bifurcating structure of the main and accessory portions of the former muscle is more advanced in Ornithorhynchus, and the vestigial state of the latter muscle in Ornithorhynchus also looks like a secondary reduction. So, we regard the condition of Tachyglossidae as the most primitive in mammals and, probably, in all synapsids.

Musculus subcoracoideus (Figs. 10, 11).

Origin (Fig. 19). In Zaglossus, the muscle originates from the dorsal (internal) surface of the procoracoid and coracoid, between the origin of the m. supracoracoideus (positioned on the procoracoid more laterally) and the insertions of the $m$. sternocoracoideus and $m$. costocoracoideus (positioned more medially). The origin bifurcates running along the two longitudinal crests on the procoracoid.

In Tachyglossus, the origin of this muscle is generally similar, but reaches the caudal angle of the coracoid fusing here with the origin of the m. coracobrachialis brevis.

In Ornithorhynchus, the muscle originates from the entire dorsal surface of the procoracoid, except for a narrow medial zone, where the $m$. sternocoracoideus arises. The complete fusion of the origin of this muscle with that of the $m$. coracobrachialis brevis at the procoracoid-coracoid boundary suggests their close affinity.

Insertion (Fig. 21). In Zaglossus, the insertion of this muscle on the humerus is tendinous and descends from the lesser tubercle (which proximal apex is occupied by insertion of the m. subscapularis) along its crest, between insertions of the $m$. coracobrachialis brevis (positioned ventrally) and the $m$. teres major (positioned dorsally).

In Tachyglossus, the muscle inserts in general as in Zaglossus. The terminal aponeurosis is formed inside the distal one-fourth of the muscular belly and, having come out of it, gives a tendon, which slides over the proximal apex of the lesser tubercle of the humerus. For lower friction, the tubercle is covered by hyaline cartilage and bears a synovial capsule.

In Ornithorhynchus, the muscle inserts on the lesser tubercle and its crest by four limbs with flat tendons 
overlying each other. The most proximal tendon inserts on the proximal pole of the sesamoid formed in the terminal tendon of the $m$. subscapularis. The next tendon inserts at the distal point of insertion of the $m$. subscapularis. Two remaining tendons fuse to insert between the insertions of the $m$. coracobrachialis brevis and the $m$. teres major and, then, pass into an aponeurosis extending up to the distal end of the crest of the lesser tubercle.

Remarks. The muscle in question is difficult to separate at the origin from the $m$. coracobrachialis brevis. More than that, in Ornithorhynchus, the last spreads from the ventral onto the dorsal (internal) side of the coracoid over its caudolateral border. Howell (1937) failed to divide this muscular mass of Ornithorhynchus into the $m$. subcoracoideus and the $m$. coracobrachialis brevis and called them together the $m$. coracobrachialis profundus, because he noticed only the ventral nerve supply thereof, typical to the coracobrachial muscles but not to the subcoracoscapular ones. Panyutina et al. (2015) described a similar muscle in colugos and named it the $m$. coracobrachialis profun$d u s$ too. Like the $m$. subcoracoideus of monotremes, that muscle of colugos is positioned more dorsally (deeper, as a specimen is dissected from the ventral side) than the usual $m$. coracobrachialis longus and $m$. coracobrachialis brevis. Both of them are present in these archaic placentals and, so, their m. coracobrachialis profundus is the third and as such may represent a pure homologue of the $m$. subcoracoideus absent in any other therian.

\section{Ventral muscles of shoulder joint}

Musculus coracobrachialis brevis (Figs. 6, 7, 12).

Origin (Fig. 19). In Zaglossus, the muscle originates from the ventral (external) side of the caudolateral border of the coracoid except for its tip, where the $m$. coracobrachialis longus arises.

In Tachyglossus, the muscle originates from the ventral side of the caudolateral border of the coracoid just cranial to the $m$. coracobrachialis longus and $m$. subcoracoideus.

In Ornithorhynchus, the muscle originates from the ventral side of the caudolateral border of the coracoid (except for its tip, where the $m$. coracobrachialis longus arises) and from its dorsal (internal) surface up to the coracoid-procoracoid boundary, where it adjoins the origin of the $m$. subcoracoideus.

Insertion (Fig. 21). In Tachyglossidae, the muscle inserts fleshy throughout the proximal ventral surface of the humerus from the crest of the lesser tubercle (posteriorly) almost to the pectoral crest (anteriorly), and almost from the attachment of the articular capsule of the shoulder joint (proximally) to the insertion of the $m$. coracobrachialis longus (distally).

In Ornithorhynchus, the insertion of this muscle also occupies the whole proximal ventral surface of the humerus, but is not adjoined distally by the insertion of the $m$. coracobrachialis longus, which is crowded out distally, towards the entepicondylar foramen, by the proximal shift of insertions of the $m$. latissimus dorsi from the entepicondylus to the pectoral crest.

Remarks. In Tachyglossidae, this muscle is separated from the $m$. coracobrachialis longus by a neurovascular bundle and, in Ornithorhynchus, by the terminal tendon of the $m$. latissimus costalis, which attachment is displaced proximally up to the pectoral crest.

Musculus coracobrachialis longus (Figs. 6, 7, 11-13).

Origin (Fig. 19). In Zaglossus, the muscle originates from the ventral side of the caudal tip of the coracoid.

In Tachyglossus, it originates by a tendon from the ventral side of the caudal tip of the coracoid; then, the tendon splits into three aponeuroses: superficial, middle (entering the belly), and deep, which extends for almost one-fourth of the muscular belly length.

In Ornithorhynchus, it originates by a narrow tendon from the ventral side of the caudal tip of the coracoid; then, the tendon passes onto the external (ventral) side of the muscular belly as a superficial aponeurosis.

Insertion (Fig. 21). In Zaglossus, the muscle inserts on a distinctly outlined triangular area on the ventral side of postaxial border of the humerus, which occupies distal one-third of the bone up to the base of the entepicondylus.

In Tachyglossus, the muscle inserts in a distinctly outlined fossa on the ventral side of postaxial border of the humerus, which occupies distal half of the bone from the end of the pectoral crest to the base of the entepicondylus.

In Ornithorhynchus, the muscle bears a short terminal aponeurosis on the inner (turned to the humerus) surface of the belly. The insertion descends along a narrow line which continues the pectoral crest on the ventral side of the base of the entepicondylus, at the level of the entepicondylar foramen.

Musculus supracoracoideus ( $=$ m. infraspinatus of Diogo \& Abdala, 2010) (Figs. 6, 7, 10, 12).

Origin (Fig. 19). In Zaglossus, the origin of this muscle occupies the cranial part of the ventral surface of the procoracoid and, through a gap between its cranial border and interclavicle, comes onto the dorsal side of the two bones.

In Tachyglossus, the origin of this muscle occupies the cranial part of the ventral surface of the procoracoid and, through a gap between its cranial border and interclavicle, comes onto its dorsal side.

In Ornithorhynchus, the origin of this muscle occupies almost the entire ventral surface of the procoracoid and, through a gap between its cranial border and interclavicle, comes slightly onto its dorsal side.

Insertion (Fig. 21). In Zaglossus, the muscle inserts mostly on the anteroproximal part of the proximal ven- 
tral surface of the humerus anterior to insertion of the $m$. coracobrachialis brevis, whence, along the anterior side of the greater tubercle, it comes between insertions of the $m$. infraspinatus (positioned proximally) and the $m$. pectoralis (positioned distally) onto the dorsal side of the greater tubercle.

In Tachyglossus, the muscle only inserts on the anteroproximal part of the proximal ventral surface of the humerus between insertions of the $m$. coracobrachialis brevis (positioned posteriorly) and the $m$. infraspinatus (positioned anteriorly), without coming onto the anterior and dorsal sides of the greater tubercle.

In Ornithorhynchus, the muscle inserts mostly fleshy along the dorsal, but not ventral, side of the pectoral crest, descending from its proximal apex (the greater tubercle) to the insertion of the $m$. acromiodeltoideus.

Musculus supraspinatus (Figs. 6, 7, 9, 10).

Origin (Fig. 20). In Zaglossus, this muscle originates from the lower half of the internal surface of the scapula. Anterior fibres descend along the scapula (vertically) and posterior fibres are almost perpendicular to it (subhorizontal).

In Tachyglossus, this muscle originates from the lower one-third of the internal surface of the scapula and is fused by superficial fasciae with adjacent muscles, i.e., the $m$. levator scapulae anteriorly and the $m$. subscapularis posteriorly.

In Ornithorhynchus, this muscle is very small and its origin is restricted to the internal side of the base of the acromion just below the insertion of the $\mathrm{m}$. omohyoideus.

Insertion (Fig. 21). In all monotremes, the muscle inserts on the proximal apex of the greater tubercle of the humerus.

Remarks. The vestigial appearance of this muscle in Ornithorhynchus implies that its original mammalian condition, both size and position, is rather represented by Tachyglossidae. It is quite reasonable, indeed, that its secondary reduction in Ornithorhynchus was caused by invasion of the $m$. subscapularis onto the inner surface of the scapula, which did not yet reach this nominal position in Tachyglossidae.

Musculus infraspinatus $(=m$. teres minor of Diogo \& Abdala, 2010) (Figs. 6, 7, 10).

Origin (Fig. 20). In all monotremes, the muscle originates from the external surface of the scapula, occupying almost the entire area cranial to the crest for the origin of the $m$. triceps longus.

Insertion (Fig. 21). In Tachyglossidae, the muscle inserts on the greater tubercle of the humerus just distal to the $m$. supraspinatus.

In Ornithorhynchus, the muscle is bipennate; the fibres converge from anterior and posterior onto the flat terminal aponeurosis, which plane is perpendicular to the scapular blade. The insertion is tendinous, positioned on the greater tubercle just distal to that of the $m$. supraspinatus.
Remarks. Howell (1937) did not find the necessary supracoracoid innervation of this muscle and decided that it is the $m$. teres minor, which decision destroyed the consistent homologization of all surrounding muscles between monotremes and therians. The problem is farfetched, indeed. Both the earlier (Westling, 1889; McKay, 1894) and the later (Shrivastava, 1962) authors succeeded in finding the supracoracoid nerve supply, and so, this muscle is definitely the $m$. infraspinatus - by position, size and innervation (however, its axillary fibres are treated as the incorporated $m$. teres minor by Shrivastava, 1962). The generally accepted idea (Romer, 1922) that the therian $\mathrm{m}$. infraspinatus together with the $m$. supraspinatus are homologous to the $m$. supracoracoideus of the lower tetrapods is based mainly on similar innervation but does not take into account the fact that, in monotremes, all the three muscles are present simultaneously. More probably, the $m$. supracoracoideus of monotremes is completely homologous to the $m$. supracoracoideus of the recent reptiles, amphibians and birds, while the $m$. infraspinatus and $m$. supraspinatus are specifically mammalian add-ons to this muscular group, i.e., the advanced feature of mammalian evolutionary lineage.

\section{Dorsal muscles of humerus}

Musculus triceps brachii $(=m$. anconeus) is, in fact, composed of five heads. Two scapular heads are the caput longum pars superficialis, and c. longum $p$. profundus, and three humeral heads are the caput laterale, c. mediale, and c. accessorium. For brevity, we call them the $m$. triceps longus superficialis, $m$. triceps longus profundus, $m$. triceps lateralis, $m$. triceps medialis, and $m$. triceps accessorius.

Musculus triceps longus superficialis et profundus (Figs. 5, 6, 11, 13).

Origin (Fig. 20). In all monotremes, both long heads of triceps originate by a superficial aponeurosis from a special crest, which descends along the middle of the lower part of the external surface of the scapula to the glenoid. In Zaglossus, the origin of the $m$. triceps longus profundus descends further curving caudal to the glenoid and closely approaching the coracoid.

Insertion (Fig. 22). In all monotremes, both long heads of triceps run to the apex of the olecranon; the $m$. triceps longus superficialis inserts on its posterior projection (which is more than half of its extent in Zaglossus and about one-fourth in Tachyglossus and Ornithorhynchus) and the $m$. triceps longus profundus inserts almost throughout its extent from the posterior to anterior projection.

Remarks. Since the $m$. triceps longus superficialis originates from the scapula somewhat cranial to the $m$. triceps longus profundus and inserts on the olecranon more posteriorly, the two heads pull the ulna in a crisscross manner; this may help operate peculiar ball-andsocket elbow joint of monotremes (similar joint is only 
found in anurans). In therians characterized by a hingelike elbow joint, there is, usually, no such division of the $m$. triceps longus into two, and, hence, their long head may be regarded as homologous to both long heads of monotremes together. Otherwise, it may be homologous to the $m$. triceps longus profundus alone. Indeed, mustelids possess the second two-joint head of this muscle originating above the usual caput longum on the dorsocaudal angle of the scapula (Ercoli et al., 2015). This, so-called, caput angulare looks somewhat similar to the $m$. triceps longus superficialis of monotremes. If they are really homologous, the doubleheaded scapular triceps should be regarded as a primitive muscular set of mammals as a whole.

Musculus triceps lateralis (Fig. 6).

Origin (Fig. 21). In Zaglossus, this head originates by a narrow tendon a little proximal to the centre of the proximal dorsal surface of the humerus, more precisely, between the proximal extremities of attachment areas of the $m$. clavodeltoideus and $m$. brachialis radialis.

In Tachyglossus, this head originates fleshy from the proximal dorsal surface of the humerus more proximally than in Zaglossus, just distal to attachment of the shoulder joint capsule, posterior to the origin of the $m$. brachialis radialis, and just anterior to that of the $m$. triceps accessorius.

In Ornithorhynchus, this head originates by a narrow tendon from the proximal dorsal surface of the humerus more anteriorly, from a special tubercle positioned between the insertion of the $m$. infraspinatus and the origin of the $m$. brachialis.

Insertion (Fig. 22). In Tachyglossidae, this head inserts on the anterior projection of the olecranon apex.

In Ornithorhynchus, it inserts fleshy on the anterior projection of the olecranon apex almost fusing by the posterior side with the $m$. triceps accessorius.

Remarks. This head is separated from the other four by a neurovascular bundle.

Musculus triceps medialis et accessorius (Figs. 6, $11,13)$.

Origin (Fig. 21). In Tachyglossidae, these two heads are united at their origin, being separated more distally by a neurovascular bundle. The $m$. triceps accessorius arises fleshy from the proximal dorsal surface of the humerus posterior to the origin of the $m$. brachialis radialis and almost up to the crest of the lesser tubercle, while the $m$. triceps medialis arises, also fleshy, just distal to the $m$. triceps accessorius from the distal dorsal surface of the humerus.

In Ornithorhynchus, the origins of the two heads are similar to those of Tachyglossidae but are completely separate from each other.

Insertion (Fig. 22). In all monotremes, these two heads insert on the humerus-facing aspect of the olecranon, the $m$. triceps accessorius - along its apical border, from anterior to posterior projection, and the $m$. triceps medialis - on the surface between this border and the elbow joint facet of the ulna. In Tachyglossidae, the insertion of the $m$. triceps accessorius is aided by aponeurosis on the posterior side, and the insertion of the $m$. triceps medialis is aided by aponeurosis on the anterior side.

Remarks. These two heads are separated from each other by a neurovascular bundle but, at the insertion, they are difficult to separate. Together, they are distinct from the $m$. triceps lateralis and, hence, the $m$. triceps accessorius may be regarded as a proximal subdivision of the $m$. triceps medialis. Similar subdivision is well known among therians.

Musculus epitrochleoanconeus medialis $(=m$. epitrochleoanconeus of Diogo \& Abdala, 2010) (Figs. $5,6,11,13)$.

Origin (Fig. 21). In Tachyglossidae, the muscle originates from the dorsal surface of the entepicondylus, close to its tip.

In Ornithorhynchus, it originates from the dorsal surface of the tip of the entepicondylus.

Insertion (Fig. 22). In all monotremes, the muscle inserts on the posterior projection of the olecranon apex.

Remarks. This muscle distinctly differs from the $m$. triceps medialis by direction and is separated from it by nerves converging to the entepicondylar foramen (Fig. 13). It is apparently homologous to the $m$. flexor antebrachii ulnaris of lower tetrapods (Jouffroy et al., 1971).

Musculus epitrochleoanconeus lateralis (=m. anconeus of Diogo \& Abdala, 2010) (Figs. 5, 6, 11, 13).

Origin (Fig. 21). In all monotremes, the muscle originates from the dorsal surface of the ectepicondylus of the humerus anterior (preaxial) to the $m$. triceps medialis.

Insertion (Fig. 22). In Zaglossus, the muscle inserts along the anterior border of the olecranon and further descends the ulna somewhat distal to the elbow joint.

In Tachyglossus, the insertion of this muscle occupies the anterior border of the olecranon but does not descend distal to the elbow joint; it is marked by rugosity.

In Ornithorhynchus, the insertion of this muscle is similar to that in Zaglossus.

Remarks. This muscle is apparently homologous to the $m$. extensor antebrachii ulnaris of lower tetrapods (Haines, 1939).

\section{Ventral muscles of humerus}

Musculus biceps brachii (Figs. 7, 12) has two heads which are obviously homologous to the therian $m$. biceps brachii caput longum and c. breve. For brevity, we call them the $m$. biceps longus and $m$. biceps brevis.

Origin (Fig. 19). In Zaglossus, the m. biceps longus originates just caudal to the $m$. supracoracoideus from the ventral side of the procoracoid. The $m$. biceps brevis originates just caudal to the $m$. biceps longus 
from the procoracoid and adjacent region of the lateral border of the stem of the interclavicle.

In Tachyglossus, the $m$. biceps longus originates just caudal to the $m$. supracoracoideus from the ventral side of the procoracoid, penetrating under the lateral border of the stem of the interclavicle. The $m$. biceps brevis originates just caudal to the $m$. biceps longus from the procoracoid and coracoid.

In Ornithorhynchus, the $m$. biceps longus originates just caudomedial to the $m$. supracoracoideus from the ventral side of the medial border of the procoracoid and, a little, from the dorsal side of the lateral border of the stem of the interclavicle, which overhangs the medial border of the procoracoid from below. The $m$. biceps brevis originates more caudally, from the ventral side of the caudal tip of the coracoid just cranial to the $m$. coracobrachialis longus.

Insertion (Fig. 22). In Tachyglossidae, the m. biceps longus and $m$. biceps brevis pass side by side to the posterior surface of the antebrachium, at its midlength. The $m$. biceps longus inserts by a narrow aponeurosis just distal to the $m$. brachialis ulnaris on the radial edge of the ulna (Allen (1912) claimed that, in Zaglossus, there is no ulnar insertion of the biceps muscle). Some fibres of the $m$. biceps longus may traverse to the terminal aponeurosis of the $m$. biceps brevis. The $m$. biceps brevis descends more distally and inserts by a wider aponeurosis on the radius just distal and closer to the ulna than the $m$. brachialis radialis.

In Ornithorhynchus, distal to the level of the pectoral crest of the humerus, the $m$. biceps longus fuses with the $m$. biceps brevis to insert together with it on the posterior surface of the radius at its midlength, distal to the $m$. brachialis radialis. So, as compared to Tachyglossidae, the $m$. biceps longus has shifted its insertion from ulna onto radius. However, its part in the common area of insertion on the radius remains proximal to that of the $m$. biceps brevis.

Remarks. In contrast to therians, the $m$. biceps brevis is bigger in monotremes than the $m$. biceps longus.

Musculus brachialis (Figs. 7, 12) has two heads which can be termed the $m$. brachialis caput ulnare and c. radiale, according to their insertions on the antebrachium. For brevity, we call them the $m$. brachialis ulnaris and $m$. brachialis radialis. Allen (1912) took the latter for the $m$. brachioradialis, although noticed its tight junction with the former.

Origin (Fig. 21). In Zaglossus, the origin of the $m$. brachialis radialis invades the proximal dorsal surface of the humerus wedging proximally between the origins of the $m$. triceps lateralis (which is more anterior) and the $m$. triceps accessorius (which is more posterior). From this point it descends along a tiny ridge (just anterior to it), which represents the so-called anterior dorsoventral line of the humerus (Fig. 2). This term was introduced in the first publication of Romer (1922), and we keep it, although it cannot be understood now in its proper sense, since Romer, later, abandoned his initial idea of the ventral nature of deltoid muscles, which attach to the same surface of the humerus anterior to the $m$. brachialis. The origin of the $m$. brachialis ulnaris is located anterior and distal to the origin of the $m$. brachialis radialis, even coming a little from the proximal dorsal surface of the humerus onto the distal ventral one over the preaxial side of the humerus. According to Romer (1922), the origin on the distal ventral surface should be regarded as primitive condition of the $m$. brachialis among tetrapods, and invasion onto the proximal dorsal surface, as advanced feature of synapsids.

In Tachyglossus, the two heads are most separate from each other. They originate from the proximal dorsal surface of the humerus side by side, the $m$. brachialis ulnaris being placed anterior to the $m$. brachialis radialis. The latter approaches the humeral head anterior to the origin of the $m$. triceps lateralis, in contrast to Zaglossus and Ornithorhynchus.

In Ornithorhynchus, the two heads are least separate from each other. They originate together from the proximal dorsal surface of the humerus, widely separating the origin of the $m$. triceps lateralis (which is more anterior) from the origin of the $m$. triceps accessorius (which is more posterior).

Insertion (Fig. 22). In Tachyglossidae, the m. brachialis ulnaris inserts on the radial edge of the posterior surface of the ulna, just distal to the elbow joint. The $m$. brachialis radialis inserts just distal to the $m$. brachialis ulnaris on the posterior surface of in the radius, descending almost to its midlength.

In Ornithorhynchus, insertions of both heads are positioned similarly to those of Tachyglossidae but the extent of insertion of the $m$. brachialis radialis is smaller. Notable is the groove on the ulna for the flat terminal tendon of the $m$. brachialis ulnaris.

Remarks. Since, in monotremes, the intra-antebrachial mobility is extremely reduced, the double-headed state of the $m$. brachialis should be regarded as the remnant of an earlier evolutionary stage, where this division was somehow excused by the primitive freedom of movement of the radius relative to the ulna.

\section{Dorsal muscles of antebrachium and manus}

Musculus supinator (Fig. 14).

Origin (Fig. 21). In Zaglossus, the muscle originates fleshy from the entire ventral surface of the ectepicondylus of the humerus, from under the other antebrachial extensors, which arise from the ectepicondylar border. The anterior side of the muscle bears an aponeurosis of origin, which descends almost up to the midlength of its belly.

In Tachyglossus, it originates from the ventral side of the ectepicondylus with the help of two aponeuroses covering the anterior and anteromedial sides of the belly; some fibres arise from the proximal one-fourth of the medial aponeurosis of origin of the $m$. extensor digitorum communis. 
In Ornithorhynchus, it originates from the ventral side of the ectepicondylus mostly fleshy, but is aided by an aponeurosis on the anterolateral side.

Insertion (Fig. 22). In Zaglossus, the muscle inserts fleshy on the preaxial side of the proximal three-quarters of the radius, somewhat apart from the elbow joint.

In Tachyglossus, it inserts fleshy on the anteropreaxial side of the distal two-thirds of the radius, somewhat apart from the wrist joint.

In Ornithorhynchus, it inserts fleshy on the anteropreaxial of the proximal half of the radius, somewhat apart from the elbow joint.

Musculus brachioradialis (Fig. 14).

Origin (Fig. 21). In Zaglossus, the muscle originates fleshy from the ventral surface of the ectepicondylus near its proximal border, deeper than the $m$. extensor carpi radialis.

In Tachyglossus, it originates fleshy from the ventral surface of the ectepicondylus along its proximal border; only postaxial part of the origin comes under the origin of the $m$. extensor carpi radialis.

In Ornithorhynchus, it originates fleshy from the ventral surface of the ectepicondylus apart from its proximal border, deeper than the $m$. extensor carpi radialis and also the $m$. extensor digitorum communis, which has expanded proximally along the border of the ectepicondylus.

Insertion (Fig. 23). In Tachyglossidae, the terminal tendon of this muscle passes under the $m$. abductor pollicis longus to insert on the radiale + intermedium + centrale, namely, on its dorsal projection between its joints with the distal carpals II and III. The terminal tendon is held at the distal end of the radius by a connective tissue sheath.

In Ornithorhynchus, the only considerable difference from Tachyglossidae is that the terminal tendon of this muscle is bound to the distal end of the radius by a transverse branch of the terminal tendon of the $m$. abductor pollicis longus.

Musculus extensor carpi radialis (Fig. 14).

Origin (Fig. 21). In Zaglossus, the muscle originates from the ventral side of a special crest (crista supraectepicondylaris, Fig. 2) developed along the proximal border of the ectepicondylus of the humerus.

In Tachyglossus, it originates from the ventral side of the proximal as well as preaxial border of the ectepicondylus.

In Ornithorhynchus, it originates from the ventral surface of the ectepicondylus parallel to its proximal border, deeper than the m. extensor digitorum сотmunis.

Insertion (Fig. 23). In Zaglossus, the terminal tendon passes under the m. abductor pollicis longus, under a transverse retinaculum ligament binding it to the distal end of the radius, and then splits into three branches, which insert on the dorsal side of the bases of metacarpals II-IV. The branch to the metacarpal III is the thickest and that to metacarpal IV is sometimes absent.

In Tachyglossus, the terminal tendon passes in the same way as in Zaglossus, but the branch to metacarpal II is considerably thinner and that to metacarpal IV is absent (however, it was described by Haines, 1939).

In Ornithorhynchus, the terminal tendon passes under the $m$. abductor pollicis longus, but over the transverse branch of the terminal tendon of the latter muscle. There is no transverse retinaculum ligament, which binds the tendon of the $m$. extensor carpi radialis to the radius in Tachyglossidae. There are three terminal branches, which insert on the dorsal side of the bases of metacarpals II-IV.

Remarks. The insertion, being centred on the metacarpal III, is surprisingly symmetrical relative to the mid-axis of the metacarpus. This makes this muscle of monotremes comparable with the reptilian $m$. extensor digitorum communis ( $=m$. humerodorsalis of Haines, 1939), rather than with the reptilian $m$. extensor carpi radialis, which terminates more proximally and preaxially.

Musculus abductor pollicis longus (Fig. 14).

Origin (Fig. 22). In Zaglossus, the origin of this muscle occupies adjacent parts of the anterior side of the radius and ulna, descending from the elbow joint for more than a half of the radius length.

In Tachyglossus, the muscle originates similarly to that of Zaglossus, but on the ulna only. More postaxially, the anterior surface of the ulna is occupied by the origin of the $m$. extensor pollicis et indicis, and the boundary between the areas of origin of the two muscles is marked on the bone by a special rugosity.

In Ornithorhynchus, it originates from the anterior side of the proximal end of the radius and adjacent part of the ulna.

Insertion (Fig. 23). In Zaglossus, the terminal tendon of this muscle is formed in the distal one-fourth of the antebrachium and passes under its own transverse retinaculum ligament in a special groove on the distal end of the radius to the preaxial side of the metacarpal I.

In Tachyglossus, the muscular belly bears distal aponeuroses on its inner and outer surfaces, which two fuse to form the terminal tendon passing under its own transverse retinaculum ligament at the distal end of the radius to the preaxial side of the metacarpal I.

In Ornithorhynchus, the terminal tendon passes under its own transverse retinaculum ligament at the distal end of the radius to the preaxial side of the base of metacarpal I. At the level of the wrist joint, the main tendon gives off a postaxial branch, which passes over the terminal tendon of the $m$. brachioradialis, but deeper than all the other extensor tendons, and inserts on the preaxial side of the ulnare.

Musculus extensor pollicis et indicis $(=m$. extensor digitorum profundus of Haines, $1939=m$. extensor pollicis longus $+m$. extensor indicis $+m$. extensor 


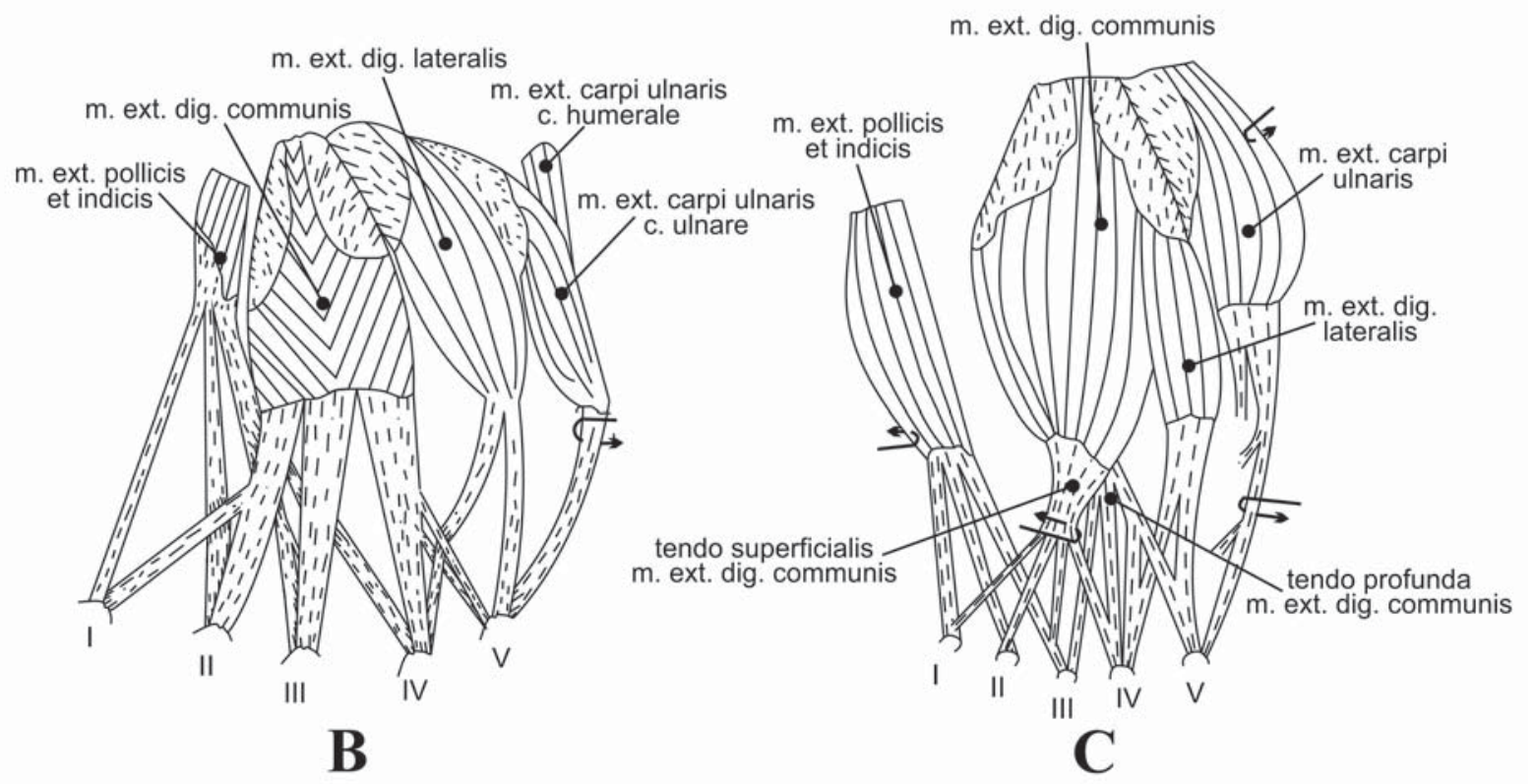

Figure 15. Interrelations of forearm extensors and composition of digital extensor tendons. Spread-view diagram.

digitus III proprius of Diogo \& Abdala, 2010) (Figs. 13-15).

Origin (Fig. 22). In Zaglossus, the muscle originates from the anterior surface of the ulna, except for its distal end and the olecranon; a small tubercle is developed at the proximal point of the area of origin.

In Tachyglossus, it originates from the anterior surface of the ulna, excluding its distal end but including the base of the olecranon. Also, the belly receives a small muscular bundle from the $m$. extensor digitorum communis.

In Ornithorhynchus, the muscle arises mainly from the olecranon: the origin descends from its anterior projection, along the anterior side of the crest of the ulna, to the level of the elbow joint and somewhat distal to it.

Insertion (Fig. 23). In Zaglossus, a flat tendon is formed as a superficial aponeurosis covering distal two-thirds of the belly. The tendon passes under the retinaculum extensorum (transverse ligament connecting distal ends of the ulna and radius), deeper than the tendon of the $m$. extensor digitorum communis, the two tendons being separated by a special bridge of the retinaculum extensorum. There are three terminal branches which insert on the ungual phalanges of the digits II-IV immediately preaxial and proximal to respective tendinous branches of the $m$. extensor digitorum communis.

In Tachyglossus, there are not only terminal branches to the ungual phalanges of the digits II-IV, found in Zaglossus. In addition, there is a branch to the ungual phalanx of the digit I, which joins at the insertion the respective tendon of the m. extensor digitorum communis. More than that, before division into the branches to the ungual phalanges, the internal (ventral) surface of the common tendinous plate of the $m$. extensor pollicis et indicis sends deeper branches to the bases of preungual phalanges of the digits II-IV (these collateral insertions are not shown on Fig. 23B1).

In Ornithorhynchus, a flat tendon is formed as a superficial aponeurosis covering the distal half of the belly. The tendon passes under the retinaculum extensorum, deeper than the tendon of the $m$. extensor digitorum communis, and splits into three terminal branches to the ungual phalanges of the digits I-III, shared with respective tendinous branches of the $m$. extensor digitorum communis.

Remarks. Of the three patterns of insertion, i.e., on the digits II-IV in Zaglossus, on I-IV in Tachyglossus, and on I-III in Ornithorhynchus, we regard the condition I-IV as the most primitive. In placentals, this muscle is usually divided into two, namely, the $m$. extensor pollicis longus and the m. extensor indicis, of which the latter often retains tendon to digit III and even to digit IV.

Musculus extensor digitorum communis (Figs. 14, 15 ) is the common superficial extensor of the digits and can be also treated as the $m$. extensor digitorum superficialis.

Origin (Fig. 21). In Zaglossus, the muscle originates from the ventral side of the preaxial border of the ectepicondylus, deeper than the $m$. extensor carpi ulnaris, with which it shares the aponeurosis of origin spreading over the entire area of contact of the outer side of the $m$. extensor digitorum communis and inner side of the $m$. extensor carpi ulnaris $c$. humerale. Also, this aponeurosis gives rise to the $m$. extensor digitorum lateralis.

In Tachyglossus, it originates from the ventral side of the distal-preaxial angle of the ectepicondylus, deeper than the m. extensor carpi ulnaris, by two superficial 
aponeuroses on the inner and outer sides of the belly. The outer aponeurosis is shared with the $m$. extensor carpi ulnaris $c$. humerale and $m$. extensor digitorum lateralis and forms a longitudinal trough accommodating the belly of the last muscle.

In Ornithorhynchus, its origin on the ventral side of the ectepicondylus ascends from the crest on its preaxial apex (here this muscle arises under the $m$. extensor carpi ulnaris) along the entire proximal border (here it arises over the $m$. extensor carpi radialis). There are two aponeuroses of origin: the wider one covers the inner and anterior sides of the belly, and the narrower one on its outer side is shared with the m. extensor carpi ulnaris and $m$. extensor digitorum lateralis.

Insertion (Fig. 23). In Zaglossus, the muscle forms before the wrist joint a flat terminal tendon, which passes under the retinaculum extensorum, and then, on the dorsal surface of the manus, strongly expands and splits into three branches which insert on the ungual phalanges of the digits II-IV. In one specimen, before branching, the common tendon fused with that of the $m$. extensor pollicis et indicis, so that they shared the three terminal branches to the ungual phalanges of the digits II-IV. Also in that specimen, the terminal branch to the digit IV sent an accessory sub-branch to the tendon of the $m$. extensor digitorum lateralis inserting on the digit $\mathrm{V}$, while the terminal branch to the digit II had a thin connection with the dorsal superficial fascia of the digit I. In fact, in that case, there was a single extensor of all five digits, which humeral and ulnar heads were homologous to the normal $m$. extensor digitorum communis and $m$. extensor pollicis et indicis, respectively.

In Tachyglossus, some muscular fibres split off from the belly to join the $m$. extensor pollicis et indicis, while the majority of fibres converge on an internal aponeurosis which shortly comes out of the belly as a flat terminal tendon; having passed under the retinaculum extensorum, it splits into three main branches to the digits II-IV. In addition, the main branch of the digit II sends a minor sub-branch to the ungual phalanx of the digit I, and the main branch of the digit IV sends a pair of minor sub-branches to the ungual phalanx of the digit $\mathrm{V}$; one of the pair passes above, and the other under the tendon of the $m$. extensor digitorum lateralis to the digit IV.

In Ornithorhynchus, the terminal tendon is formed in the distal one-third of the antebrachium, passes under the retinaculum extensorum, and then, on the manus, divides into two sheets, superficial and deep. The superficial sheet splits into four branches to the ungual phalanges of the digits I-IV, while the deep sheet splits into three branches to the ungual phalanges of the digits III-V. Before the insertion on the ungual phalanges, the long tendons send, from their inner (ventral) surface, deeper and shorter branches to the bases of preungual phalanges (these insertions are not shown on Fig. 23C1) and fuse, by their outer (dorsal), surface with more superficial interdigital tendons of the $m$. cutaneus trunci.
Remarks. The pre-mammalian integrity of the digital extensors may be modelled by an individual variation in Zaglossus, where the $m$. extensor digitorum communis and $m$. extensor pollicis et indicis converged from the humerus and ulna, respectively, onto a common tendon giving branches, though unequal, to all five digits. The other trace of this ancient convergence is the slip of fibres, which, in Tachyglossus, splits off from the belly of the $m$. extensor digitorum communis and joins the $m$. extensor pollicis et indicis.

Musculus extensor digitorum lateralis $(=m$. extensor digiti minimi of Diogo \& Abdala, 2010) (Figs. 14, $15)$.

Origin. In Zaglossus, the fibres of this muscle arise from the aponeurosis of origin on the outer side of the m. extensor digitorum communis and, in two specimens of the three studied, directly (fleshy) from the ectepicondylus of the humerus.

In Tachyglossus, its fibres arise from the outer aponeurosis of origin of the m. extensor digitorum communis, which forms a longitudinal trough accommodating the belly of the $m$. extensor digitorum lateralis.

In Ornithorhynchus, this muscle is rudimentary. Its fibres arise from the outer aponeurosis of origin of the $m$. extensor digitorum communis and from the inner aponeurosis of origin of the $m$. extensor carpi ulnaris.

Insertion (Fig. 23). In Zaglossus, the terminal tendon is formed at the midlength of the antebrachium, passes in a special groove along the ulna under a separate transverse retinaculum ligament and, in the manus, bifurcates into branches to the ungual phalanx of the digit IV (at insertion, this branch fuses with respective tendon of the $m$. extensor pollicis et indicis) and to the preungual phalanx of the digit $\mathrm{V}$ (its ungual phalanx is reduced).

In Tachyglossus, the terminal tendon arises as aponeurosis on the outer side of the belly and inserts by two branches on the ungual phalanges of the digits IV and $\mathrm{V}$.

In Ornithorhynchus, the terminal tendon is very thin; it runs in a special groove along the ulna under the tendon of the $m$. extensor carpi ulnaris, passes together with it under their common transverse retinaculum ligament, and then bifurcates into branches, which become gradually thinner and, before their insertions on the ungual phalanges of the digits IV and V, fuse with respective tendons of the $m$. extensor digitorum communis.

Musculus extensor carpi ulnaris (Figs. 13-15).

Origin (Figs. 21, 22). In Zaglossus, this muscle has two heads, which are the caput humerale and c. ulnare. The humeral head originates from the apex of the ectepicondylus by the inner-side aponeurosis shared with the $m$. extensor digitorum communis and $m$. extensor digitorum lateralis. The ulnar head originates from the anterior aspect of the ulna at the level of the proximal end of the radius. 
In Tachyglossus, the humeral head originates from the apex of the ectepicondylus, even expanding onto its dorsal side, with the help of the inner-side aponeurosis shared with $m$. extensor digitorum communis and the $m$. extensor digitorum lateralis. The ulnar head originates from the anterior aspect of the olecranon up to the level of the proximal end of the radius.

In Ornithorhynchus, there is only the humeral head. Its fleshy origin on the apex of the ectepicondylus is slightly aided by the aponeurosis shared with the $m$. extensor digitorum lateralis and $m$. extensor digitorum communis.

Insertion (Fig. 23). In Zaglossus, the caput humerale and $c$. ulnare fuse together at the midlength of the antebrachium to produce a common tendon, which passes under a special transverse retinaculum ligament at the distal end of the ulna and inserts in the digit $\mathrm{V}$ by two branches: the proximal one runs to the postaxial side of its metacarpophalangeal joint, and the distal one terminates on the preaxial side of its proximal interphalangeal joint. Distal to the ulnare and up to the very insertion, the tendon is underlaid by a collagen-adipose pad.

In Tachyglossus, fibres of the two muscular heads converge in a bipennate fashion onto a common terminal aponeurosis, which comes out of the belly as a tendon. It passes under a special transverse retinaculum ligament at the end of the ulna to insert extremely distally, on the base of the ungual phalanx of the digit $\mathrm{V}$, postaxial to the $m$. extensor digitorum lateralis.

In Ornithorhynchus, the terminal tendon passes under the transverse retinaculum ligament at the distal end of the ulna, together with the tendon of the $m$. extensor digitorum lateralis, and, in the metacarpal region, splits into four branches. The three main branches insert in a cascade manner at the postaxial side of the three joints of the digit $\mathrm{V}$, while the most proximal and thin branch deviates postaxially to join the $m$. interosseus Vlateralis at the outer side of the metacarpal V.

\section{Ventral muscles of antebrachium and manus}

Musculus pronator teres (Figs. 7, 16) is the most superficial muscle on the inner surface of the antebrachium.

Origin (Fig. 21). In Zaglossus, the muscular fibres originate from the round area on the ventral side of the entepicondylus and from an aponeurosis on the posterior side of the muscle, which is shared with the $m$. flexor carpi radialis.

In Tachyglossus, the origin of this muscle on the ventral side of the entepicondylus is narrower than in Zaglossus. In addition to the aponeurosis on the posterior side, shared with the $m$. flexor carpi radialis, the second aponeurosis of origin is present on the anterior side of the belly.

In Ornithorhynchus, the origin is on the ventral side of the apex of the entepicondylus.

Insertion (Fig. 22). In Zaglossus, the muscle inserts on the preaxial side of the radius distal to its midlength.
In Tachyglossus, it inserts on the preaxial side of the distal part of the radius.

In Ornithorhynchus, the muscular belly bears terminal aponeuroses on its anterior and posterior sides, which fuse together into a flat tendon inserting on the posterior side of the radius close to its midlength.

Musculus flexor carpi radialis (Figs. 13, 16).

Origin (Fig. 21). In Zaglossus, the origin of this muscle extends along the ventral side of the entire distal border of the entepicondylus, from its apex up to the humeral condyle, being interrupted by the origin of the $m$. flexor digitorum profundus c. humerale profundum. The separation of the two zones of origin reflects bipennate structure of the belly of the $m$. flexor carp $i$ radialis. It bears an aponeurosis of origin on the inner side, which is shared with the underlying $m$. pronator teres, and an aponeurosis of origin on the outer side, which is shared with the overlying $m$. flexor digitorum profundus $c$. humerale superficiale.

In Tachyglossus, the origin is similar to that in Zaglossus, but is not interrupted by the origin of the $m$. flexor digitorum profundus c. humerale profundum. The muscular fibres are relatively shorter, and the two aponeuroses of origin shared with the $m$. pronator teres and $m$. flexor digitorum profundus $c$. humerale superficiale are more developed: they descend, roughly, one half of the length of the antebrachium or four-fifths of the length of the belly.

In Ornithorhynchus, the origin is similar to that in Tachyglossidae. However, the muscular belly is more flattened in the parasagittal plane and shows more pronounced bipennate structure. The anterior penna bears an aponeurosis of origin on the anterior side, which faces the humeral condyle and the radius. The posterior penna, bears two aponeuroses of origin, the inner one, shared with the underlying $m$. pronator teres, and the outer one, facing the overlying $m$. flexor digitorum profundus c. humerale superficiale.

Insertion (Fig. 23). In Zaglossus, muscular fibres from the inner and outer aponeuroses of origin converge in a bipennate fashion onto a common external terminal aponeurosis, which becomes a wide flat tendon running to the proximal apex of the prepollex connected with the radiale+intermedium +centrale. From the prepollex, the pull of the muscle is also transferred via ligaments to the distal carpals and metacarpals I-III.

In Tachyglossus, short muscular fibres converge from the aponeuroses of origin onto a thick terminal aponeurosis, which becomes a flat tendon running to the proximal apex of the prepollex connected with the radiale+intermedium + centrale and, by two ligaments, with the distal carpals I and II.

In Ornithorhynchus, muscular fibres converge in a bipennate fashion, from anterior and from posterior, onto the middle terminal aponeurosis, which becomes a round tendon inserting on the proximal apex of the prepollex. 

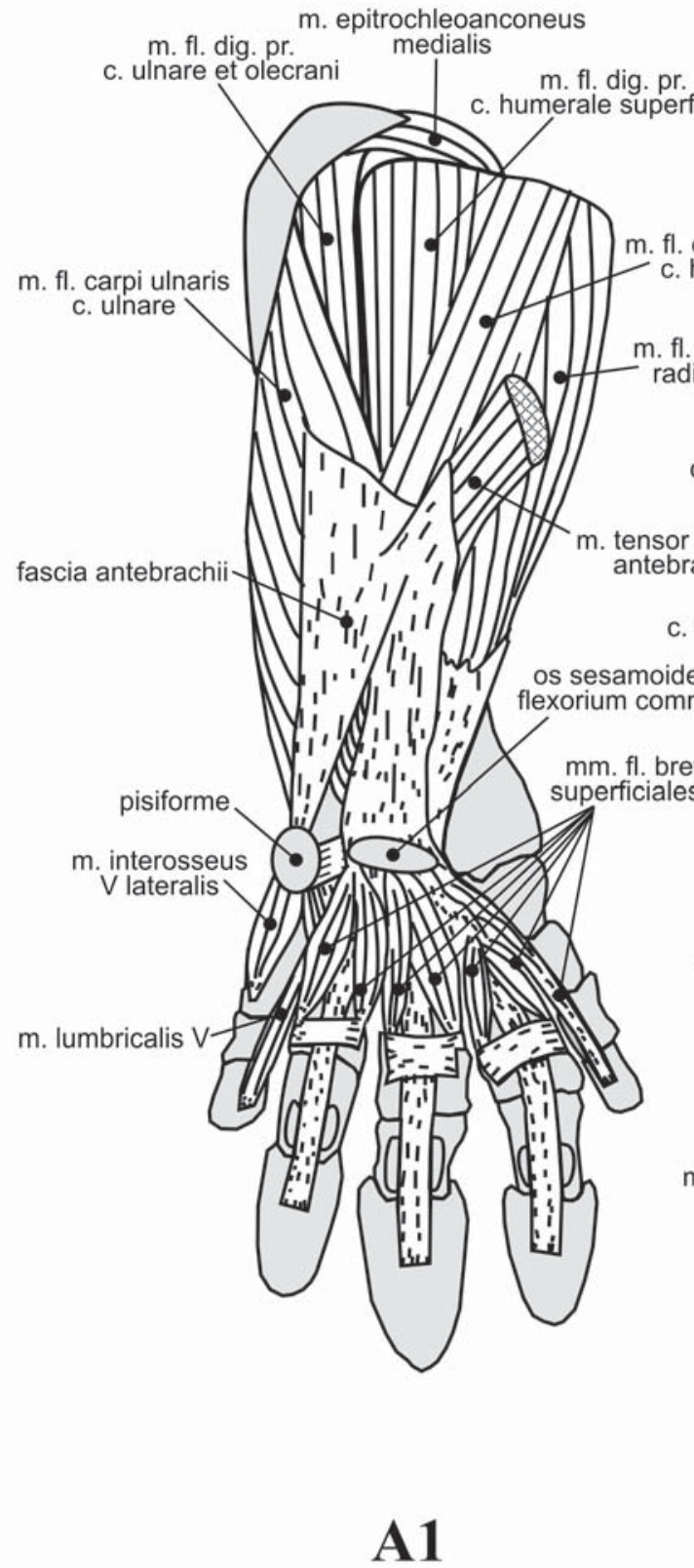

m. fl. carpi ulna c. ulnare

m. epitrochleoanconeus medialis

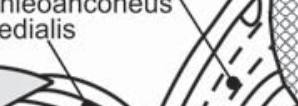
m. fl. dig. pr.
c. ulnare et olecrani

fasciae c. humerale profundum

\section{mune}

tendo $\mathrm{m}$. fl. carpi
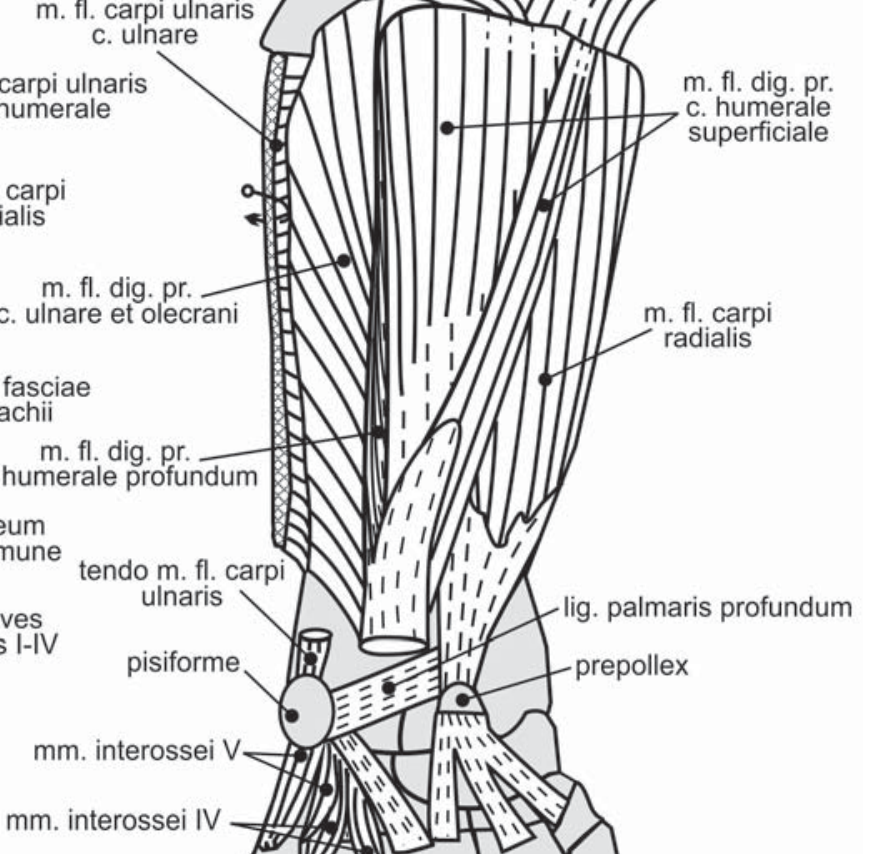

$\mathrm{m}$. interossei $\mathrm{V}$
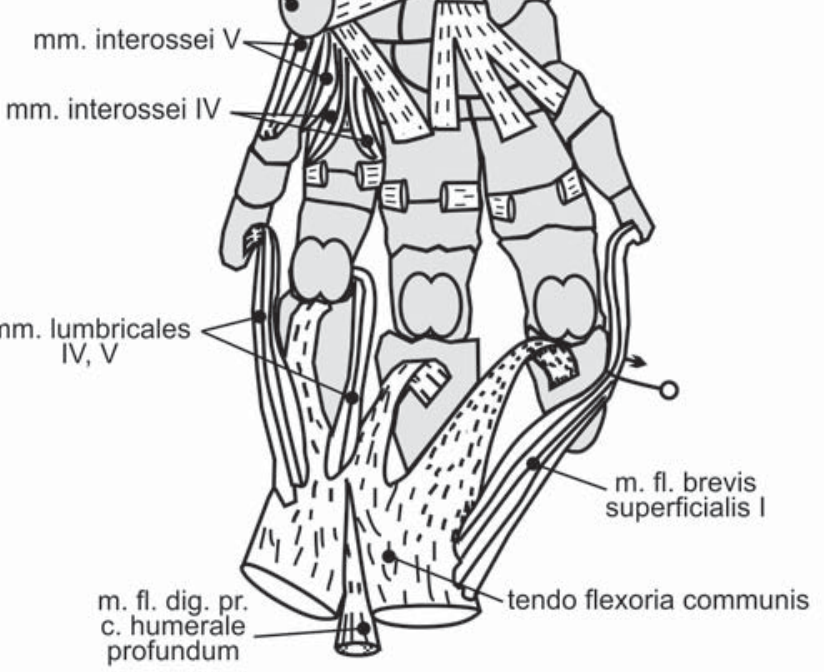

A2

Figure 16. Forearm and palmar flexors.

Remarks. The prepollex increases the lever arm of the $m$. flexor carpi radialis for flexion and medial adduction in the wrist joint. In Zaglossus, due to the distal ligaments, it plays the same role for the carpometacarpal I-III joints.

Musculus flexor digitorum profundus $(=m$. flexor digitorum longus of Diogo \& Abdala, 2010) (Figs. 13, $16,18)$ is composed of two long heads arising from the humerus, namely, the caput humerale superficiale and c. humerale profundum, and two short heads arising from the ulna, namely, the caput olecrani and c. ulnare. Of all the four heads, the most separate is the caput humerale profundum: it is distinctive by a spindleshaped belly and joins the common terminal tendon much more distally than the others.

Origin (Figs. 21, 22). In Zaglossus, the caput humerale superficiale originates from the ventral side of the distal border of the entepicondylus throughout its length with the help of two aponeuroses, of which the outer one is shared with the $m$. flexor carpi ulnaris $c$. humerale, and the inner one with the $m$. flexor carpi 


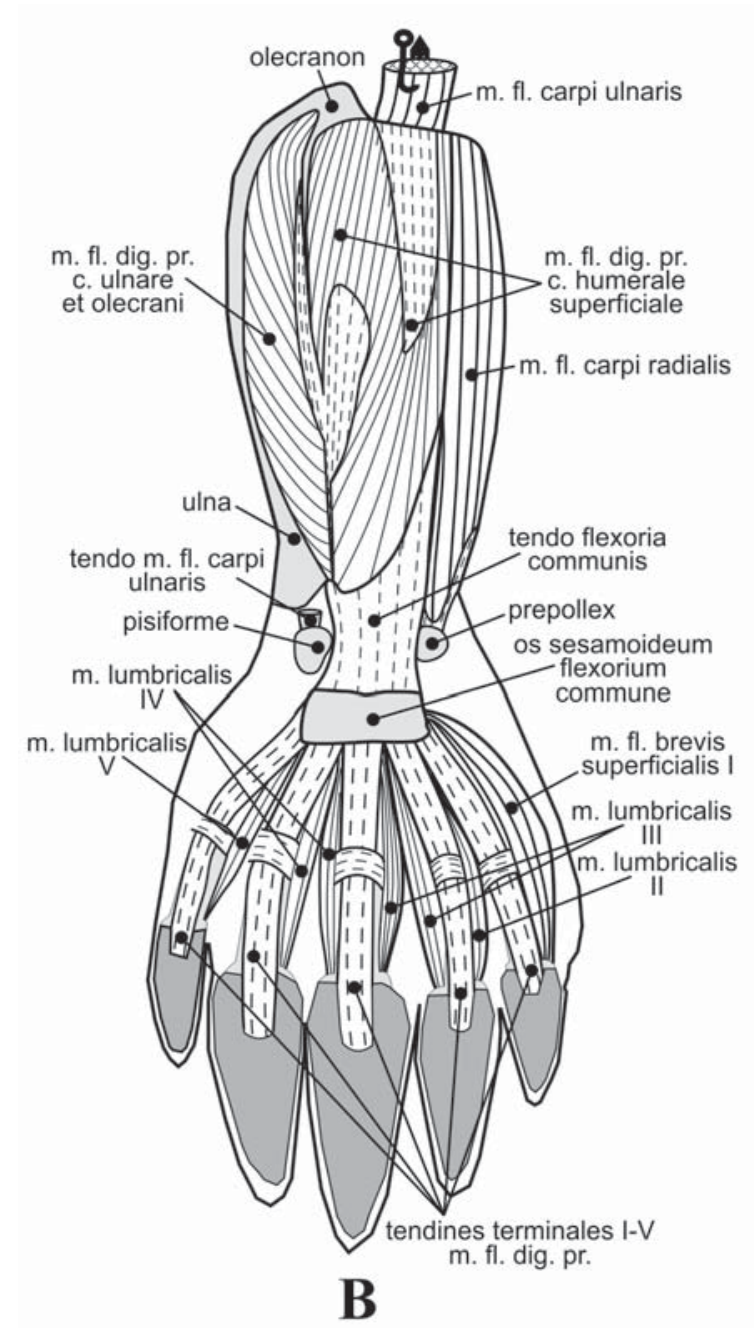

Figure 16 (continued).

radialis. The caput humerale profundum has a compact origin on the ventral side of the distal styloid process of the entepicondylus (spina entepicondyli, Fig. 2). The caput olecrani and c. ulnare are not differentiated from each other and arise together from proximal threequarters of the posterior side of the ulna, including the olecranon. In one specimen, a few deeper fibres arised not from the ulna, but close to it, from the radius (more precisely, from the distal one-fourth of its posterior side), as a presumptive rudiment of the caput radiale, found in therians.

In Tachyglossus, the origins of all heads are similar to those in Zaglossus. The origin of the united caput ulnare et olecrani is outlined on the ulna by two crests.

In Ornithorhynchus, the caput humerale superficiale is the biggest and, due to very short fibres, the strongest of the heads. It originates from the distal border of the entepicondylus. Its belly is extremely flattened in the parasagittal plane and has a bipennate structure. The two aponeuroses of origin cover the outer and the inner sides of the belly; additional layer of fibres arises from the inner side of the inner aponeuro- sis forming a third minor penna. The caput humerale profundum has long fibres originating from the ventral side of the distal styloid process of the entepicondylus. The origin of the caput olecrani and the smaller caput ulnare descends along the posterior side of the ulna from the posterior projection of the olecranon below the midlength of the bone. Within this area, the caput ulnare occupies more preaxial and distal part than the caput olecrani, and the boundary between the two on the ulna gives rise to their common aponeurosis of origin binding together the adjacent sides of the two short heads.

Insertion (Fig. 23). In Zaglossus, the caput humerale superficiale forms an awkward pennate structure. It bears two terminal aponeuroses, the outer one and the inner one. Most of its muscular fibres insert on their sides which face each other, and the outer side of the outer aponeurosis also receives a smaller bundle of fibres running from the aponeurosis of origin common with the $m$. flexor carpi ulnaris c. humerale (see above). The caput ulnare et olecrani is unipennate; its terminal aponeurosis fuses into the inner terminal aponeurosis of the caput humerale superficiale producing a thick common terminal tendon (tendo flexoria communis). The caput humerale profundum is hidden in the narrow cleft between the caput humerale superficiale and caput ulnare et olecrani, but forms its own thin terminal tendon, which runs under the common tendon of the other heads being coated from inside by the distal-most muscular fibres of the caput ulnare. This thin tendon runs separately up to the level of the wrist bend, where it fuses into the dorsal (deeper) side of the thick common tendon. In the manus, the resultant tendon splits into branches inserting on the ventral side of the bases of the ungual phalanges of the digits I-V, II-V, or IIIV. This individual variation reflects the degrees of reduction of the marginal fingers. At the branching point, the common tendon bears a so-called palmar sesamoid, which is very common across tetrapods (Ponssa et al., 2010). We introduce a new Latin term for this bone, the os sesamoideum flexorium commune, to emphasize its mechanical role in conjunction of the terminal tendons of the main flexor muscle of the digits, both in the manus and the pes.

In Tachyglossus, the pattern of insertion is in general similar to that in Zaglossus. The muscular fibres are relatively shorter, and the aponeuroses are more developed. The thin terminal tendon of the caput humerale profundum runs separately under the main tendon almost up to the os sesamoideum flexorium commune, being coated from inside by the distal-most muscular fibres of the caput ulnare, which also reach the palmar sesamoid An important difference from Zaglossus is the large size of this sesamoid embedded in the resultant tendon of all heads at the point of splitting into digital branches, which number is five. In fact, due to the large size of this sesamoid, the common tendon inserts on the proximal apex of the bone, and the branches to the ungual phalanges originate from its distal end. 


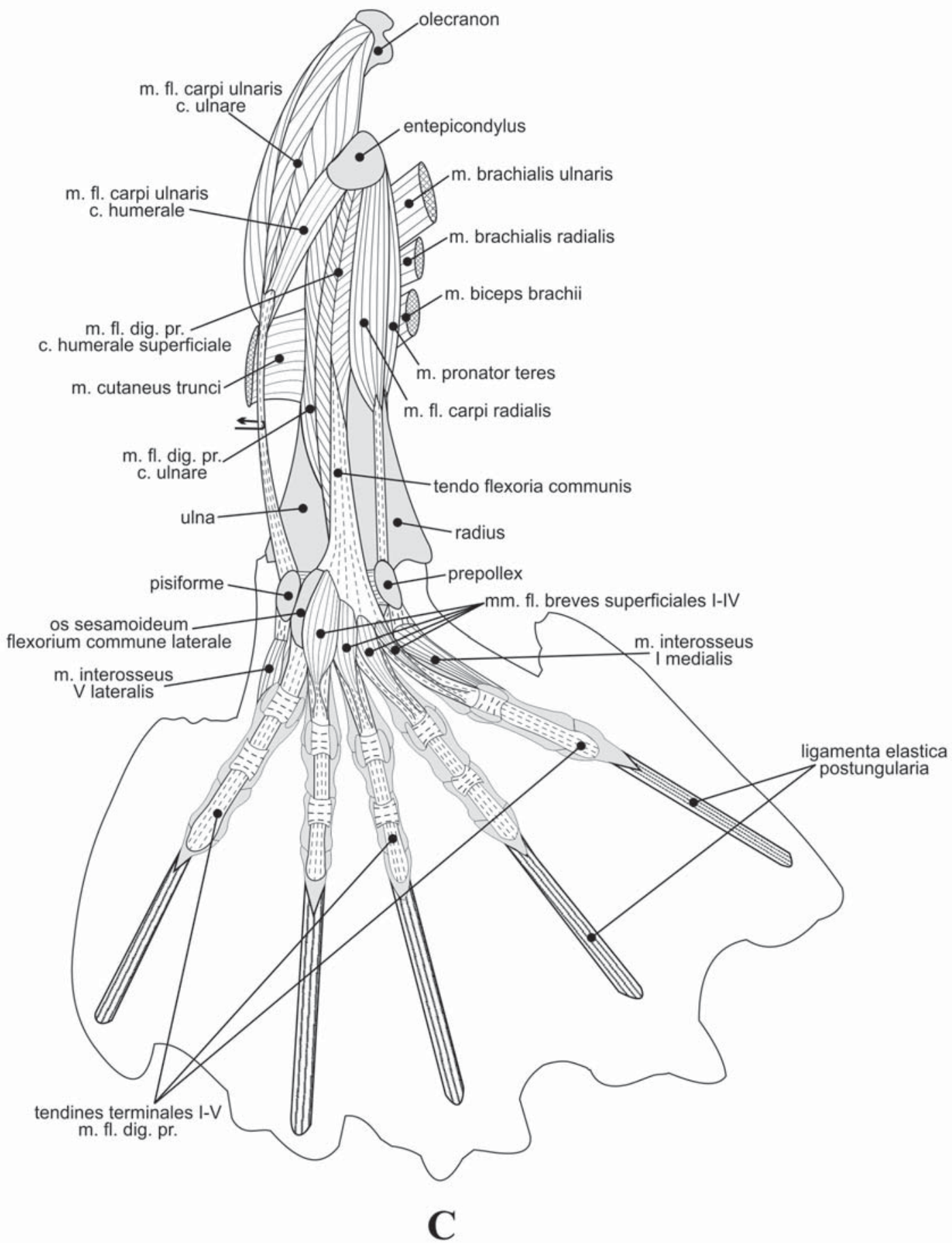

Figure 16 (continued).

To the ventral side of the sesamoid, the proximal paw pad is attached, which is composed of dense adipose tissue.

In Ornithorhynchus, the extremely numerous and short fibres of the caput humerale superficiale converge from its outer and inner aponeuroses of origin onto the first aponeurosis of insertion, which spreads in the parasagittal plane through the midst of the flat belly and forms the stem of its bipennate structure. The second, deeper terminal aponeurosis is produced by the caput ulnare together with the inner-most layer of fibres (the third penna) of the caput humerale superficiale. The caput olecrani forms the third terminal aponeurosis on its anterior side. The first aponeurosis turns 
into a very thick terminal tendon, which thickness is associated with a great force of the caput humerale superficiale (its fibres are only about $2 \mathrm{~mm}$ long, and hence, their number and the physiological cross-sectional area is extremely increased). In the distal part of the antebrachium, this central flexor tendon is joined, preaxially, by the second aponeurosis belonging to the caput ulnare and, postaxially, by the third aponeurosis belonging to the caput olecrani. The spindle-shaped caput humerale profundum squeezes down between the caput olecrani and caput ulnare, internal to the former and external to the latter, and, then, forms a thin terminal tendon, which descends to the manus along a groove on the dorsal (deeper) side of the thick common tendon of the other heads, which groove is filled with loose connective tissue. At the level of the carpus, the thick common flexor tendon bears two sesamoids, and the thin tendon of the caput humerale profundum inserts on these two separately, deeper than the common tendon. Each sesamoid carries on its dorsal side a proximodistally elongated dense adipose pad, which damps its contact with the carpus; proximally, the two pads are fused. In the centre of the dorsal side of the preaxial sesamoid (os sesamoideum flexorium commune mediale), the thick ligamentum flexorium commune transversum is inserted, which other end is attached to the ventral side of the ulnare, under the pisiforme but superficial to the ligamentum palmaris profundum. The last crosses the ventral surface of the wrist from the pisiforme and ulnare to the prepollex and incorporates a dense adipose pad at the preaxial end. Further, the preaxial sesamoid of the common flexor tendon gives rise to the terminal flexor tendons to the digits I-III, and the postaxial sesamoid (os sesamoideum flexorium commune laterale) gives branches to the digits IV and $\mathrm{V}$. The branches $\mathrm{I}$ and $\mathrm{V}$ are considerably thicker than those of the three middle digits. All the five brances insert on the bases of the ungual phalanges and are bound to the bases of all the other phalanges by subarticular ligaments. Under each metacarpophalangeal and interphalangeal joint, there is a sesamoid increasing the lever arm of the force applied through the flexor tendons. In addition to the five digital branches, the two sesamoids of the common flexor tendon give rise to four thick elastin bands which pass between the digits into the free part of the swimming membrane beyond the claws.

Remarks. The structure of the $m$. flexor digitorum profundus is most remarkable in Ornithorhynchus. It combines the traits of extreme specialization and profound primitiveness. From the functional standpoint, most important is the caput humerale superficiale, which shortened fibres ensure high forces for underwater rowing, but restrict the ability for finger extension and lead to awkward knuckle-walking on land. From the evolutionary standpoint, most interesting is the caput humerale profundum. It squeezes between the caput olecrani and caput ulnare exactly in the same way as, in urodelans, the $m$. ulnocarpalis $(=m$. flexor vetus $=m$. contrahentium caput longum) squeezes between the ventral and dorsal heads of the $m$. palmaris communis profundus sensu Miner, 1925 (which are the same as the $m$. flexor accessorius lateralis and $m$. flexor accessorius medialis of Diogo \& Abdala, 2010). It is usually accepted that the $m$. ulnocarpalis was lost beyond amphibians or as if dissolved in the $m$. flexor digitorum profundus (Straus, 1942). However, the condition found by thorough dissection in Ornithorhynchus shows that this muscle retained its identity in mammals, being shifted in its origin from the ulna onto the humerus and imprisoned inside the former $m$. palmaris communis. So, the caput humerale superficiale corresponds to the $m$. palmaris communis superficialis, and the ligamentum flexorium commune transversum connecting ulnare to the preaxial sesamoid in Ornithorhynchus is the postaxial segment of the transverse subcarpal ligament, on which the $m$. ulnocarpalis specifically inserts in urodelans. The deeper sheet of this urodelan ligament is retained as the ligamentum palmaris profundum. Note, that the ligamentum flexorium commune transversum is not unique to Ornithorhynchus: it can be found in Tachyglossus (Kajava, 1911), probably, as individual variation, and in some lipotyphlous insectivores (Howell, 1936; Straus, 1942).

Musculus flexor carpi ulnaris (Figs. 13, 16) is composed of two heads, the caput humerale and $c$. ulnare.

Origin (Figs. 21, 22). In Zaglossus, the caput humerale arises from the apex and postaxial part of the distal border of the entepicondylus and bears an innerside aponeurosis of origin shared with the underlying $m$. flexor digitorum profundus c. humerale superficiale. The caput ulnare originates from the posteriorpostaxial side of the ulna almost throughout its length, excluding the distal end but including the olecranon.

In Tachyglossus, the caput humerale originates from the dorsal side of the postaxial distal angle of the entepicondylus and is divided into two portions. The anterior portion is associated by a common aponeurosis of origin with the underlying $m$. flexor digitorum profundus c. humerale superficiale, but remains separate from the $m$. flexor carpi ulnaris $c$. ulnare up to the insertion. On the contrary, the posterior portion of the caput humerale fuses with the caput ulnare, which originates from the posterior-postaxial side of the ulna throughout its length.

In Ornithorhynchus, the caput humerale arises from the apex and postaxial part of the distal border of the entepicondylus; bearing an aponeurosis of origin on the inner side of its belly, it is unipennate. The caput ulnare has a tripennate structure, its belly is flattened in the parasagittal plane, and the origin occupies the posterior-postaxial surface of the olecranon descending distal to the elbow joint but a little.

Insertion (Fig. 23). In Zaglossus, the two heads form a common terminal aponeurosis (the input of the caput humerale is greater), which becomes a flat ten- 
don running to the proximal apex of the pisiforme. From this ossicle, three pisometacarpal ligaments descend to the bases of metacarpals III-V.

In Tachyglossus, similarly to Zaglossus, the two heads insert together on the pisiforme, wherefrom the pisometacarpal ligaments descend to the metacarpals III-V.

In Ornithorhynchus, the caput humerale bears a terminal aponeurosis on the outer side. The posterior part of this aponeurosis forms a longitudinal trough for insertion of the fibres of the caput ulnare. Thus the two heads converge, and produce a short and thick common tendon, which inserts on the proximal apex of the pisiforme. Ligaments from the pisiforme to metacarpals are absent.

Remarks. The pisiforme increases the lever arm of the $m$. flexor carpi ulnaris for flexion and lateral abduction in the wrist joint. In Tachyglossidae, due to the distal ligaments, it plays the same role for the carpometacarpal III-V joints.

Fascia palmaris manus (Fig. 17) continues the fascia antebrachii.

In Tachyglossidae, proximally, its preaxial edge is bound to the distal end of the radius, and its postaxial edge is bound to the pisiforme and the terminal aponeurosis of the $m$. flexor carpi ulnaris. Therefrom the fascia palmaris manus descends under the proximal paw pad, attaches to it and, then, splits into branches to the digital pads. In Zaglossus, there are strong branches terminating on the postaxial and preaxial sides of the pads II-IV and weaker branches to the postaxial side of the pad I and to the preaxial side of the pad V. In Tachyglossus, the branches to the pads I and V are almost as strong as the branches to the pads II-IV.

In Ornithorhynchus, proximally, its preaxial edge is bound to the distal end of the radius, and its postaxial edge is bound to the distal end of the ulna, ulnare, distal carpal IV+V, and the terminal aponeurosis of the $m$. flexor carpi ulnaris. Further distally, it becomes much thicker, attaches to the common tendon of the $m$. flexor digitorum profundus near its sesamoids, spreads over the $m$. flexores breves superficiales, and splits into branches running on either preaxial and postaxial sides of all the digits to the ungual phalanges. The preaxial and postaxial branches of the same digit are connected with each other by a thin fascia, which is fused to the underlying tendinous sheath for the digital tendon of the $m$. flexor digitorum profundus. Beyond the ungual phalanx, the pair of digital branches of the fascia palmaris manus is continued by an elastin band, which is as thick as the phalanx and reaches the distal edge of the swimming membrane.

Musculi flexores breves superficiales (Figs. 16, 18).

Origin. In Zaglossus, this series includes four preaxial flexors of the digits I-IV and, sometimes, up to

Figure 17. Subcutaneous connective-tissue structures in the palm.
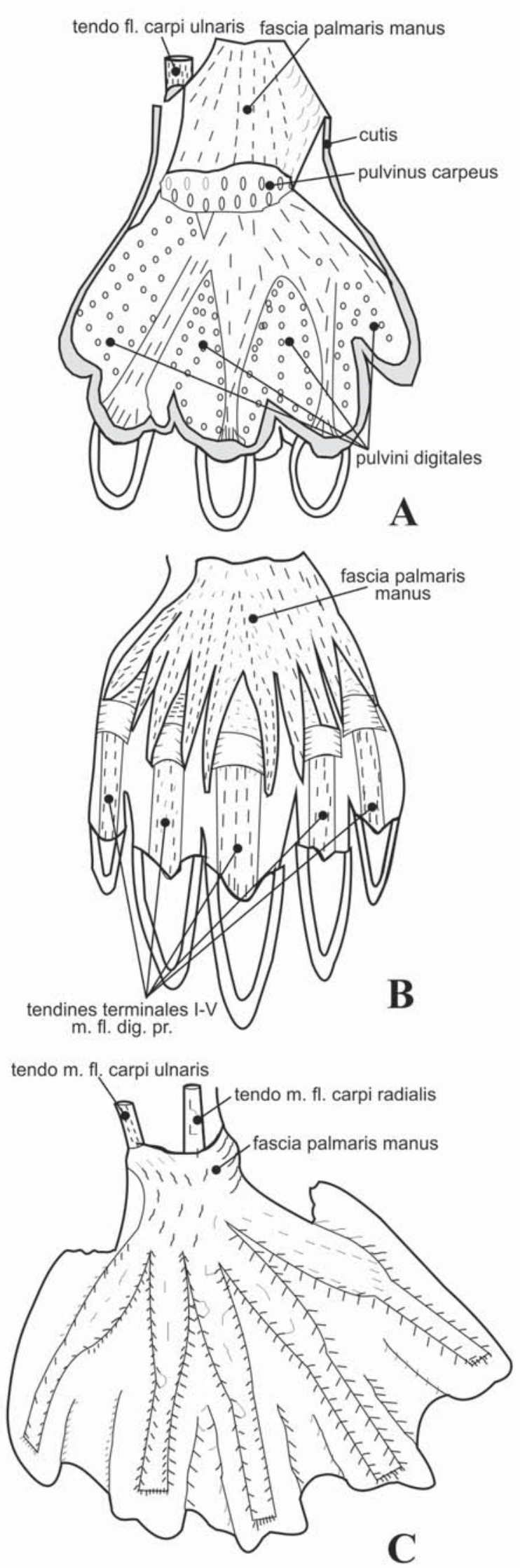


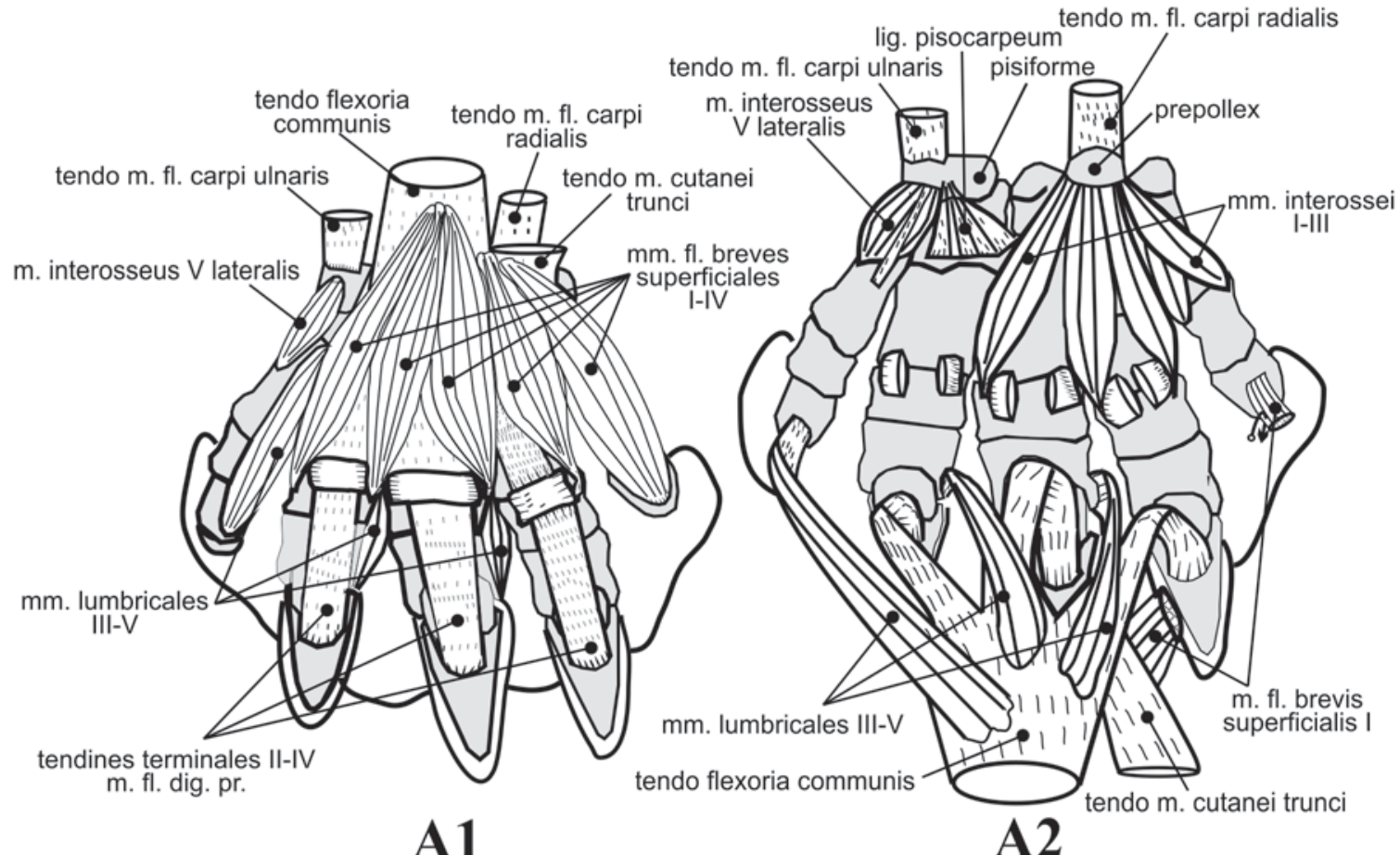

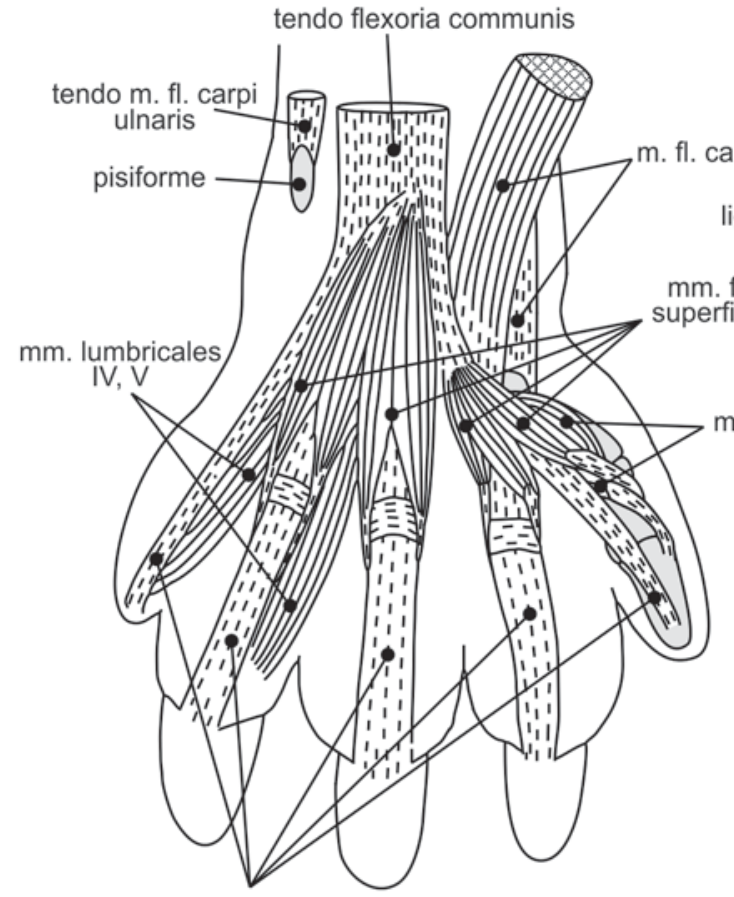

tendines terminales I-V m. fl. dig. pr.

AA1
A2 

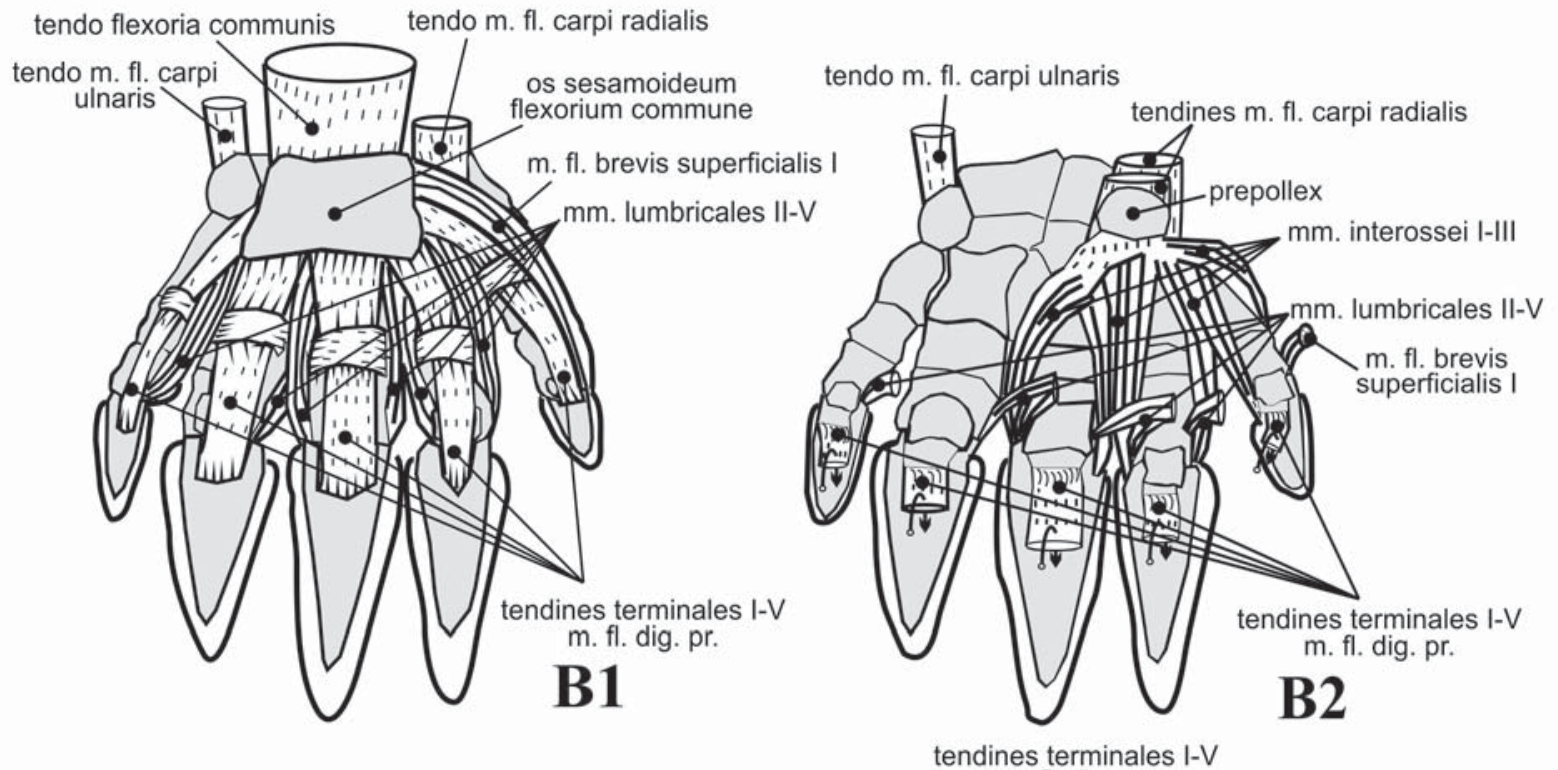

Figure 18 (continued).

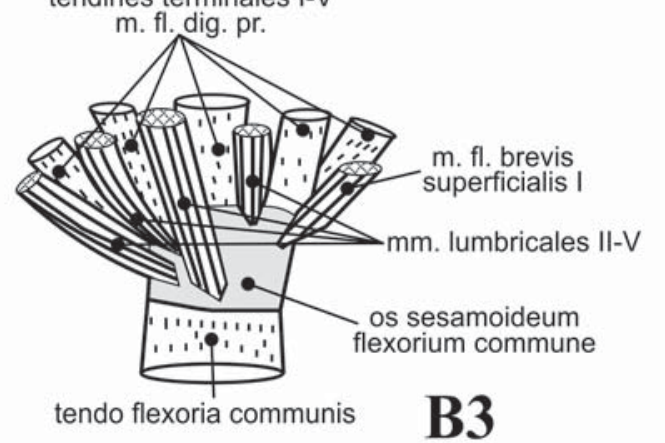

three postaxial flexors of the digits II-IV. The $m$. flexor brevis superficialis I originates from the preaxial edge, and the $m m$. flexores breves superficiales $I I-I V$ from the ventral side of the common tendon of the $m$. flexor digitorum profundus. Therefrom, the origin spreads somewhat proximally, onto the fascia palmaris manus and even onto the fascia antebrachii, so that the $\mathrm{mm}$. flexores breves superficiales I, II arise at the level of the wrist joint, and the mm. flexores breves superficiales $I I I, I V$ even at the level of the distal end of the antebrachium.

In Tachyglossus, only the $m$. flexor brevis superficialis $I$ is present, which originates from the preaxial edge and adjacent part of the dorsal side of the sesamoid of the common tendon of the $m$. flexor digitorum profundus. Alternatively, this muscle could be regarded as the $m$. lumbricalis $I$, but the thumb is never supplied by a muscle of this series among tetrapods.

Ornithorhynchus has four muscles in this series supplying the digits I-IV, which originate from the ventral side of the common tendon of the $m$. flexor digitorum profundus at the proximal border of its sesamoids.

Insertion (Fig. 23). In Zaglossus, the preaxial flexors of the digits I-IV, which are always present, pass preaxial to the respective digital tendon of the $m$. flexor

digitorum profundus and, typically, insert via metacarpophalangeal subarticular ligament at the preaxial side of the base of the phalanx 1 . This is always the case in digits II-IV, but in the digit I the insertion may be shifted distally, to the midlength of the phalanx 1 and even to its end. The postaxial flexors may be present in the digit IV and, more rarely, in the digits II and III too. They pass postaxial to the respective digital tendon of the $m$. flexor digitorum profundus and insert via metacarpophalangeal subarticular ligament at the postaxial side of the base of the phalanx 1. If both preaxial and postaxial flexors are present in a digit, they embrace the digital tendon of the $m$. flexor digitorum profundus like the branches of perforated tendon of the therian $m$. flexor digitorum superficialis.

In Tachyglossus, the $m$. flexor brevis superficialis I inserts preaxially at the end of the preungual phalanx of the thumb.

In Ornithorhynchus, the terminal tendons of the four muscles of this series become thinner and thinner sheets, which distally disappear in loose elastic tissue enveloping respective digits. In the digit I, the tendon is distinct up to the metacarpophalangeal subarticular ligament; in the digits II-IV, the tendons can be traced up to the midlengths of the first phalanges, where they embrace the respective digital tendons of the $m$. flexor 


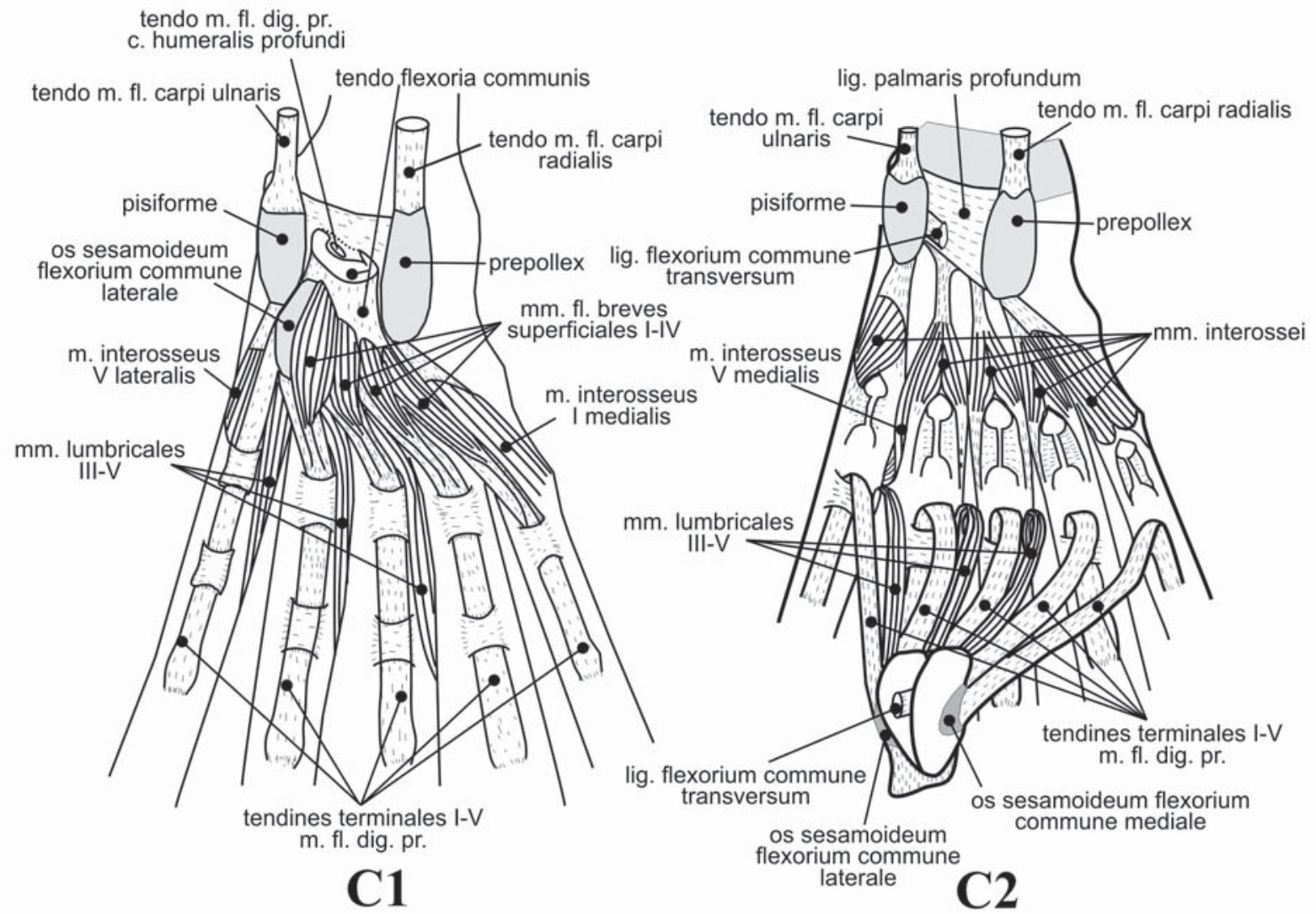

Figure 18 (continued).

digitorum profundus as very thin perforated tendons, but are not fused with them as was claimed by Diogo \& Abdala (2010).

Remarks. Due to the typical pattern of embracing the digital tendons of the $m$. flexor digitorum profun$d u s$, the evident therian homologue of this muscular series is the $m$. flexor digitorum superficialis. Despite of position of its belly in the antebrachium rather than the manus, this homology is supported by the fact, that the similar distal origin of the $m$. flexor digitorum superficialis on the terminal common tendon of the $m$. flexor digitorum profundus is sometimes found, for instance, in tupaias (Panyutina et al., 2015) and felids (Haines, 1950). In other therians, the $m$. flexor digitorum superficialis has migrated more proximally: it originates from the aponeurosis of origin of the $m$. flexor digitorum profundus or directly from the entepicondylus of the humerus.

Musculi lumbricales (Figs. 16, 18).

Origin. In Zaglossus, the muscles of this series arise from the dorsal (deep) side of the common tendon of the $m$. flexor digitorum profundus immediately proximal to its branching. The $m m$. lumbricales $I V, V$ are always present, and the $m$. lumbricalis III is rare.

In Tachyglossus, there is a set of four muscles in this series, namely, the $m m$. lumbricales $I I-V$, although the m. lumbricalis II is not always present. All of them arise from the dorsal side of the sesamoid of the common tendon of the $m$. flexor digitorum profundus.

Ornithorhynchus has three muscles in this series, which arise from the dorsal side of the common tendon of the $m$. flexor digitorum profundus at the distal border of its sesamoids. More precisely, the $m$. lumbricalis III originates from the preaxial sesamoid, the $m$. lumbricalis IV originates between sesamoids, and the m. lumbricalis $V$ originates from the postaxial sesamoid.

Insertion (Fig. 23). In Zaglossus, the insertions of these muscles, either fleshy or tendinous, are always located on the end of preungual phalanx at its preaxial side.

In Tachyglossus, there are four muscles, which insert preaxially at the end of preungual phalanges of the digits II-V. The main difference from Zaglossus is peculiar bifurcation of the $\mathrm{mm}$. lumbricales III, IV (and also $V$, according to Fewkes, 1877), which split off a preaxial head to the postaxial side of the preungual phalanx of the preceding digit. Thus, the $m$. lumbricalis III supplies the digits III and II, the $m$. lumbricalis IV supplies the digits IV and III, and the $m$. lumbricalis $V$ can supply not only the digit $\mathrm{V}$ but occasionally (Fewkes, 1877) the digit IV too. The second difference from Zaglossus is the occasional presence of the m. lumbricalis II on the preaxial side of the digit II (not found by Fewkes, 1877). 


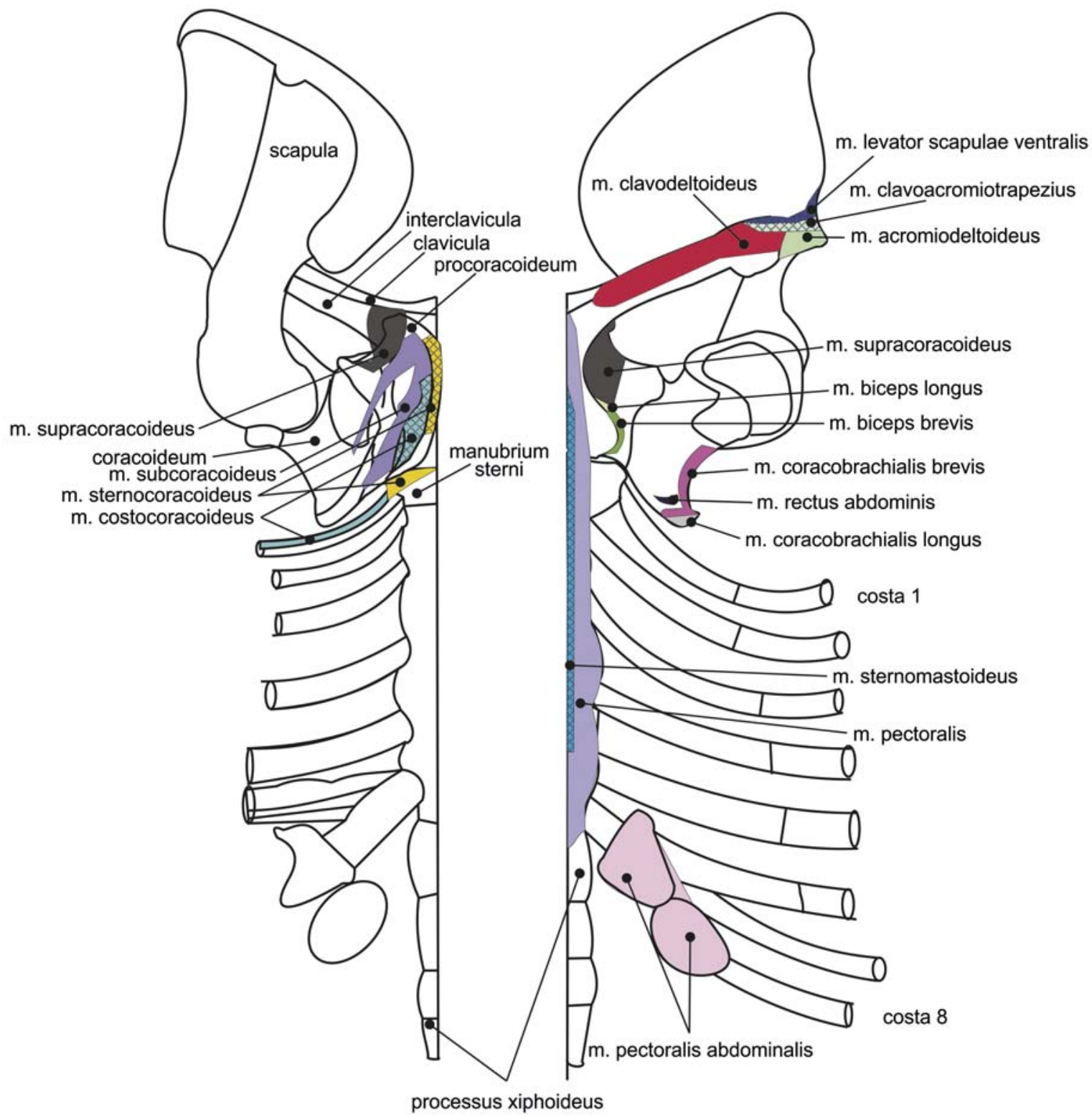

A1

A2

Figure 19. Muscular attachments on the left coracoid plate and sternum. 1 - dorsal view, 2 - ventral view.

In Ornithorhynchus, the three muscles of this series run to the preaxial side of the digits III-V, where their thin sheet-like tendons traverse, without firm attachment, over the preaxial side of the first interphalangeal joint onto the dorsal side of the respective digit to fuse into the preaxial edge of the respective digital tendon of the $m$. extensor digitorum communis. On its way, at the preaxial side of the metacarpophalangeal $\mathrm{V}$ joint, the $m$. lumbricalis $V$ fuses with the $m$. interosseus $V$ medialis.

Remarks. Bifurcation of the mm. lumbricales, which we found in Tachyglossus, is a very rare morphological case. It was only reported for colugos (Panyutina et al., 2015), which show bifurcation of the $\mathrm{mm}$. lumbricales $I I I-V$. So, these archaic therians have postaxial supply from this muscular series in the digits II-IV, alike the specimen of Tachyglossus described by Fewkes (1877).

Musculi interossei $(=m m$. flexores breves profundi of Diogo \& Abdala, 2010) (Figs. 16, 18). For muscles of the digits I and $\mathrm{V}$, special terms are applicable: $m$. interosseus I medialis $=m$. opponens pollicis, $m$. interosseus I lateralis $=m$. adductor pollicis, $m$. interosseus $V$ lateralis $=m$. opponens digiti quinti. 


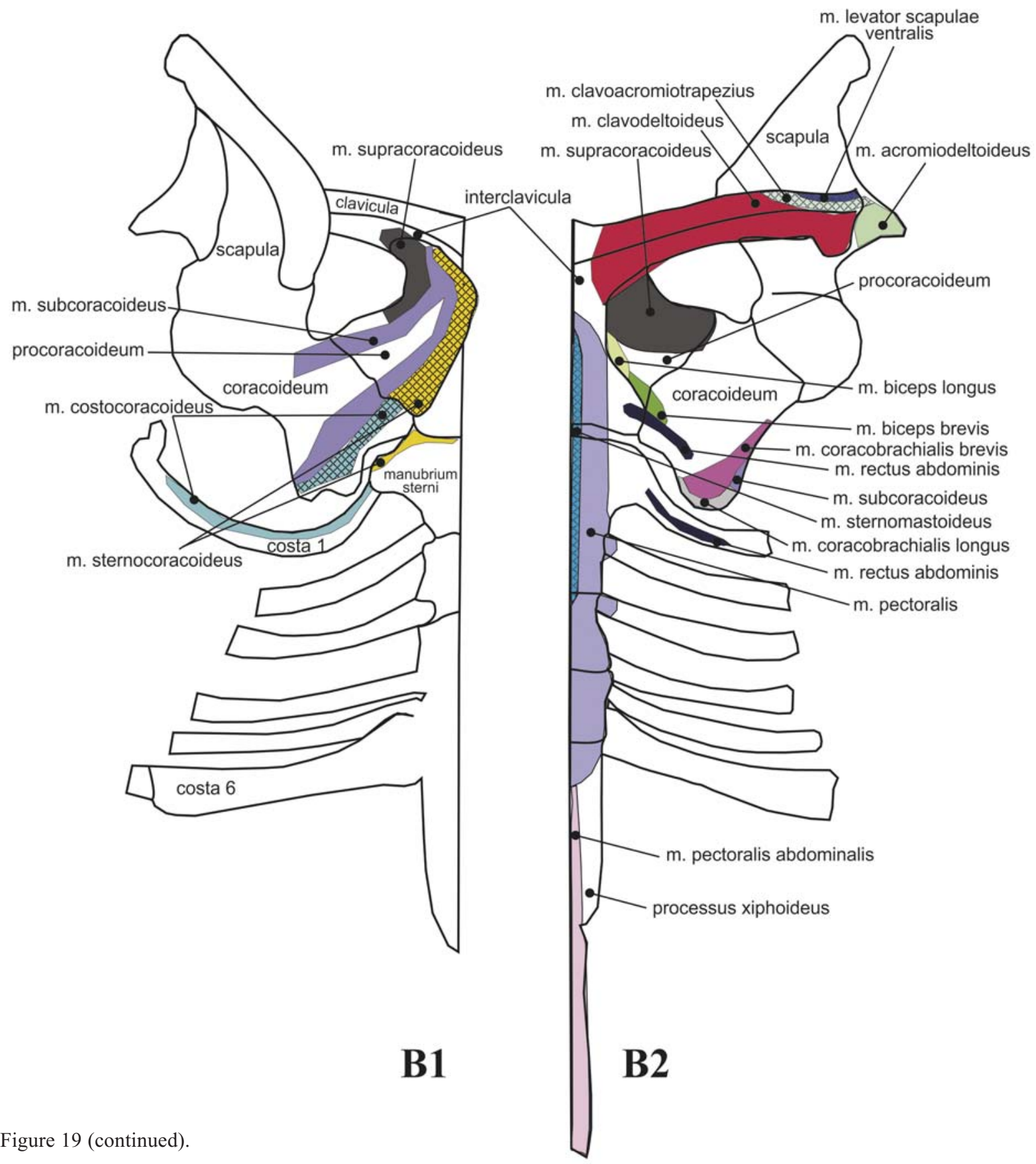

Origin (Fig. 23). In Zaglossus, the mm. interossei $I-I I I$ arise from the prepollex, while the $\mathrm{mm}$. interossei $I V, V$ originate from the ligament connecting the pisiforme to metacarpal IV, and from pisiforme itself. The muscular set is variable. Most common are the $m m$. interossei I, II mediales and the mm. interossei II, V laterales, but all the other six muscles are also possible.

In Tachyglossus, Kajava (1911) described a vast individual variation comprising, on the whole, a complete set of ten muscles (his $\mathrm{mm}$. flexores breves profundi radialis and ulnaris correspond to the $\mathrm{mm}$. in- terossei mediales and laterales, respectively). We have failed to find the $m$. interosseus IV lateralis and both of the $m m$. interossei $V$. Only five muscles were always present, namely the $m$. interosseus I medialis, the two $\mathrm{mm}$. interossei II, and the two mm. interossei III. The $m$. interosseus I lateralis and $m$. interosseus IV medialis could be present or absent. The muscles of the digits I and II originate from the prepollex (three of them, by a common tendon from its distal end, and the $m$. interosseus II lateralis - fleshy), the muscles of the digit III originate from the ligament between the prepollex and 


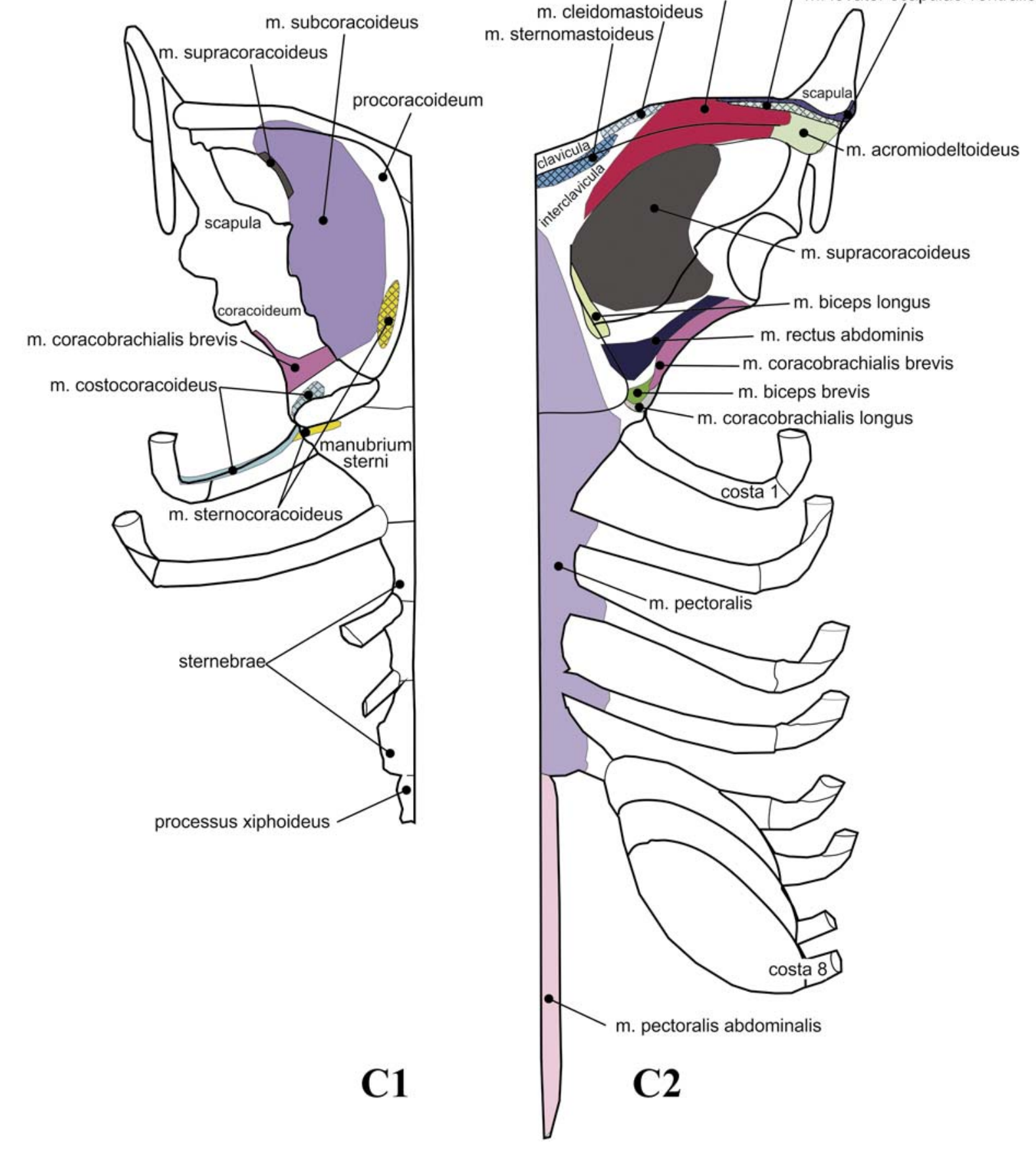

m. clavoacromiotrapezius $\mathrm{m}$. clavodeltoideus $/ \mathrm{m}$. levator scapulae ventralis

Figure 19 (continued).

the metacarpal III, and the occasional m. interosseus IV medialis arised at the pisiforme.

Ornithorhynchus has a complete set of ten muscles, that is the preaxial $m$. interosseus medialis and the postaxial $\mathrm{m}$. interosseus lateralis in every digit. In contrast to Tachyglossidae, all of them originate by long tendons, which number is five, as follows. (1) The $\mathrm{mm}$. interossei $I$ arise from the distal end of the pre- pollex; (2) the $m m$. interossei II arise deeper from the same point and from ligaments between carpals; (3) the $\mathrm{mm}$. interossei III arise from ligaments between carpals; (4) the mm. interossei $I V$ and the $m$. interosseus $V$ medialis arise from ligaments between carpals and from the ulnare; (5) the $m$. interosseus Vlateralis arises from the distal end of the pisiforme. Contrary to the other muscles of the series, the $m$. interosseus $V$ medialis 

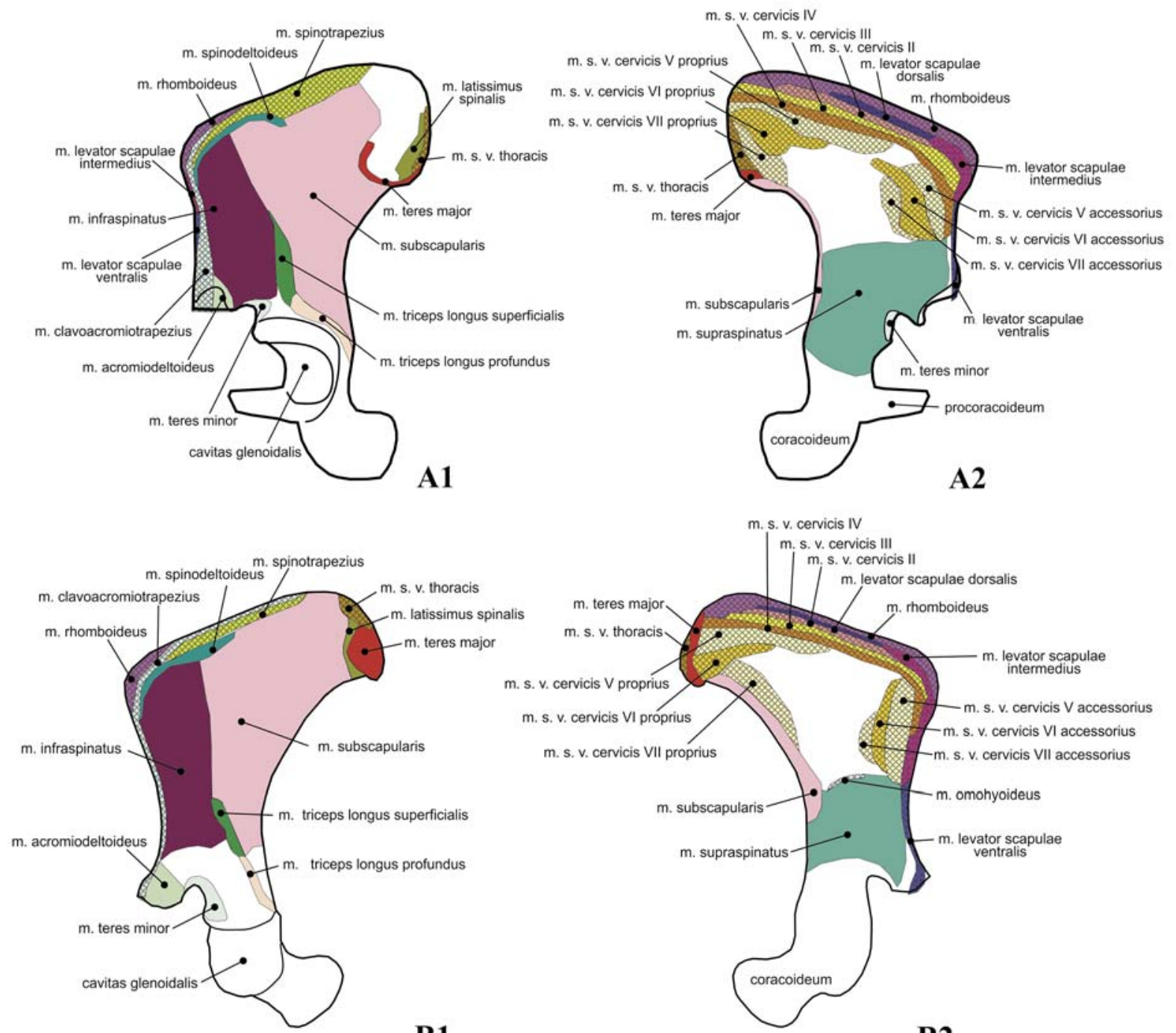

B1

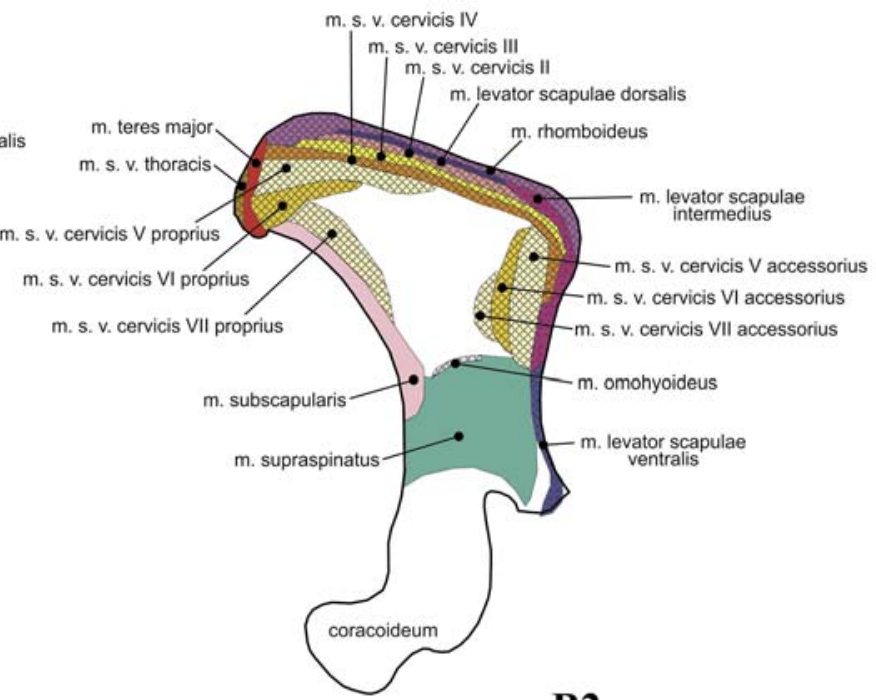

B2
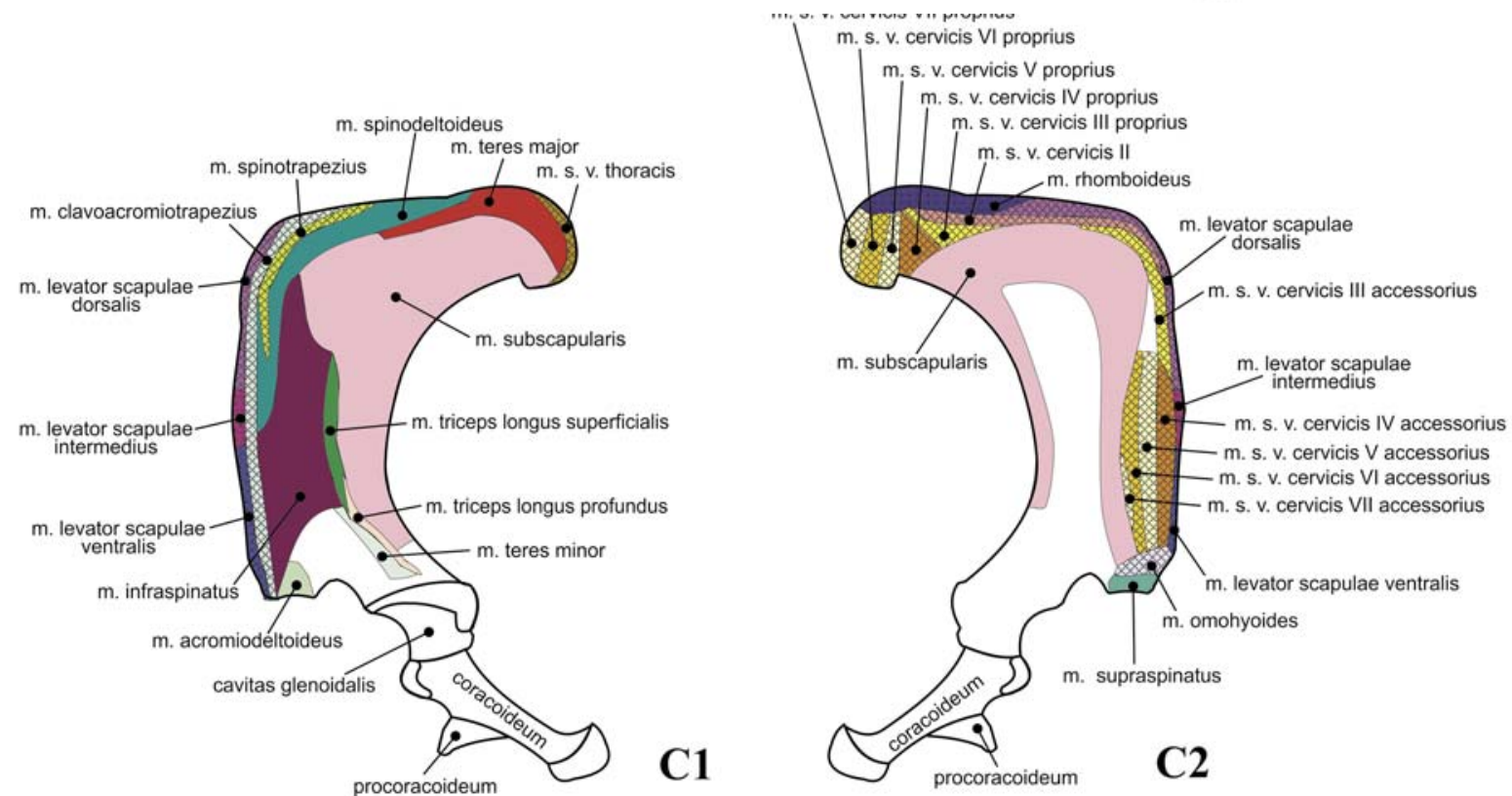

Figure 20. Muscular attachments on the left scapula. 1 - lateral view, 2 - medial view. 

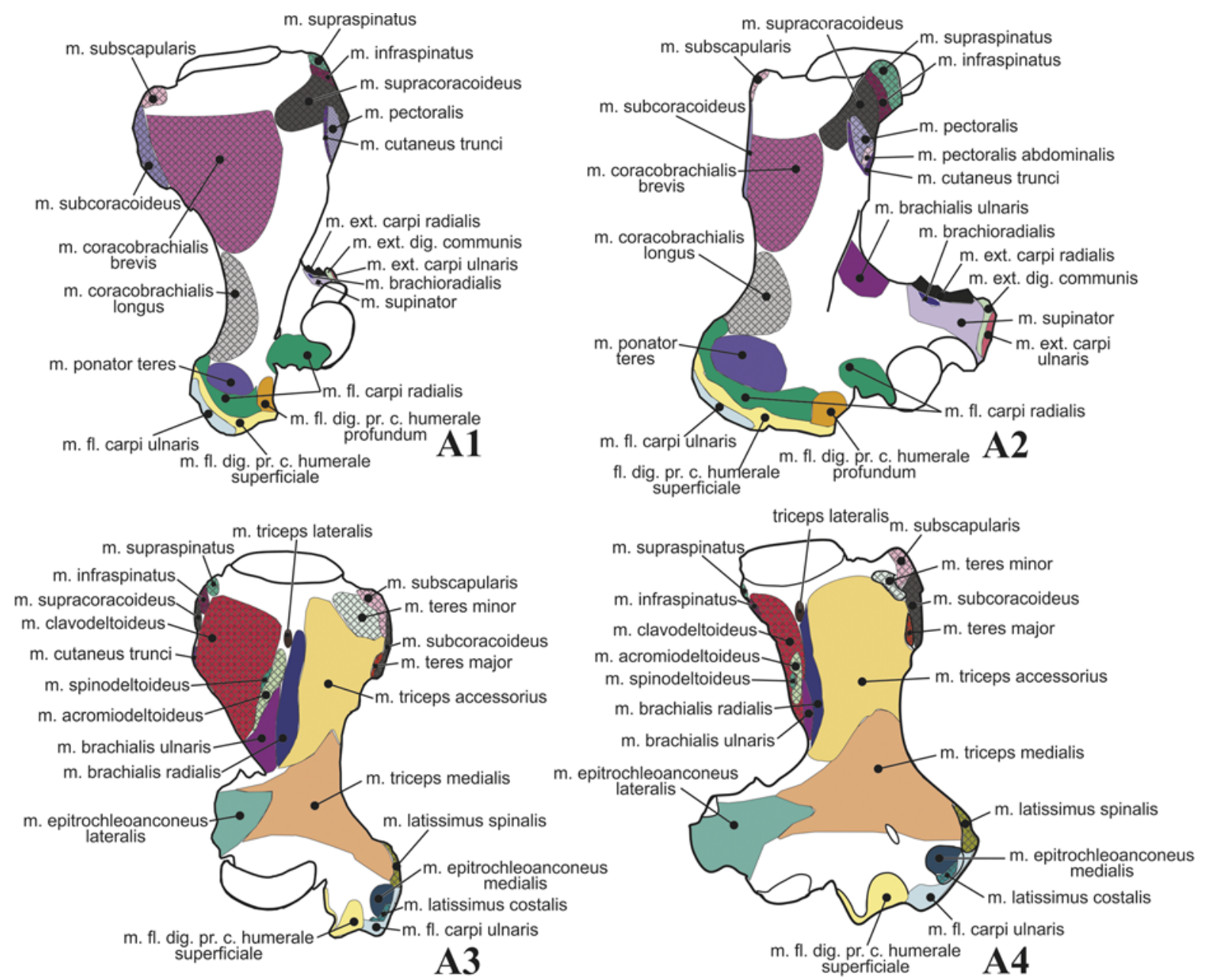

Figure 21. Muscular attachments on the left humerus. 1 - proximal ventral surface, 2 - distal ventral surface, 3 - proximal dorsal surface, 4 - distal dorsal surface. Note, that in Tachyglossidae the humerus is considerably less twisted than in Ornithorhynchus; hence, the proximal-side images $(1,3)$ are shaped more similar with the distal-side images $(2,4)$ on A and B than on $\mathrm{C}$.

shares the tendon of origin with the mm. interossei IV rather than with its twin, the $m$. interosseus Vlateralis.

Insertion (Fig. 23). In Zaglossus, these muscles insert preaxially ( $\mathrm{mm}$. interossei mediales) or postaxially (mm. interossei laterales) on the metacarpophalangeal joints. However, due to reduction of the marginal digits, the insertions of the $m m$. interossei $I$, $V$ can be shifted from this typical point more proximally, close to the bases of metacarpals I and V (Fig. 23 A2), or more distally, to the ends of these digits (this case was described by Allen (1912), who treated these marginal $m m$. interosse $i$ as the $m$. adductor pollicis and $m$. abductor digiti quinti).

In Tachyglossus, these muscles insert preaxially (mm. interossei mediales) or postaxially ( $\mathrm{mm}$. interossei laterales) on the bases of the ungual phalanges of respective digits. Only the $m$. interosseus I medialis inserts more proximally, namely, on the preaxial side of the metacarpophalangeal I joint; however, according to Fewkes (1877), it can reach the ungual phalanx of the digit I like the other muscles of the series.

In Ornithorhynchus, the fibres of the $m$. interosseus $V$ medialis are long enough to reach the proximal interphalangeal $\mathrm{V}$ joint, on which preaxial side this muscle forms a common attachment with the $m$. lumbricalis $V$. The other $m m$. interossei have shorter fibres, which only reach the level of the metacarpophalangeal joints. At this level, the $\mathrm{m}$. interosseus I medialis and $\mathrm{mm}$. interossei I, V laterales attach to respective sides of the metacarpophalangeal subarticular sesamoids; from the last, the muscular pull is transferred to the terminal parts of respective digital tendons of the $m$. extensor digitorum communis via connective-tissue sheaths of the fingers. The pull of all the other muscles of this series, is also transferred to respective extensor tendons, but in the other way - by means of special ligaments, to which the muscular fibres of the $\mathrm{mm}$. interossei II-IV mediales and laterals attach at preaxial and postaxial sides, respectively, of the metacarpophalangeal joints. There are six such ligaments passing as 


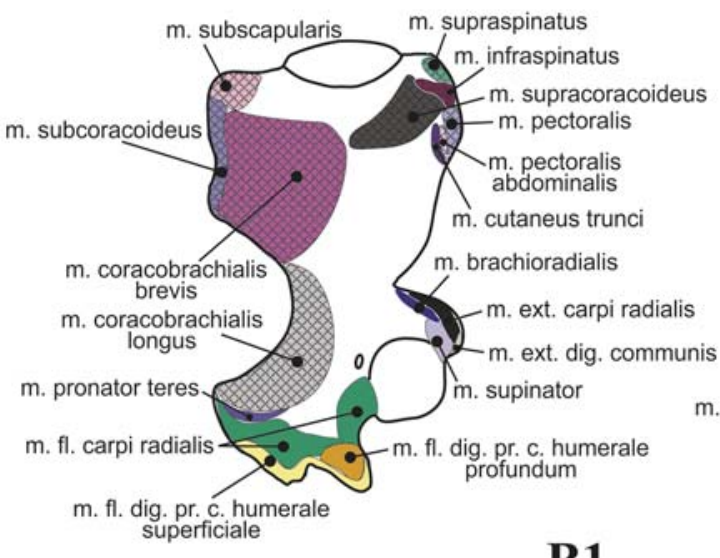

B1

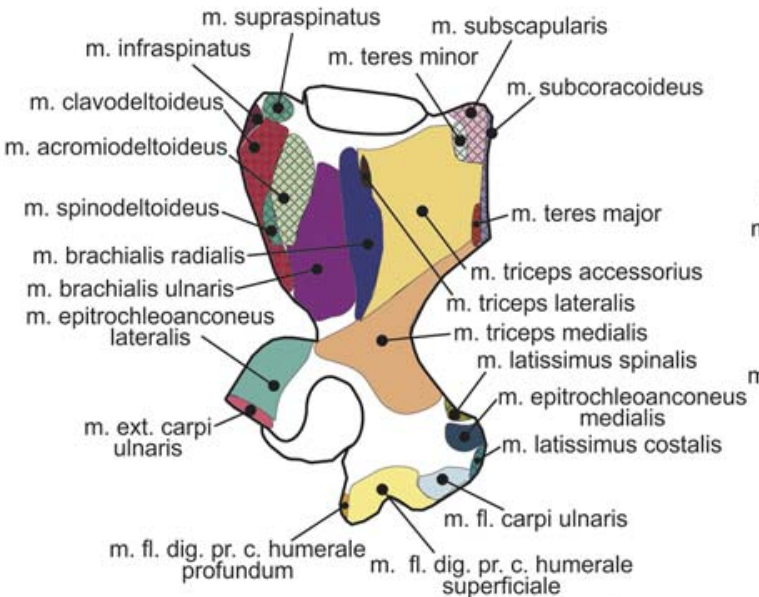

B3
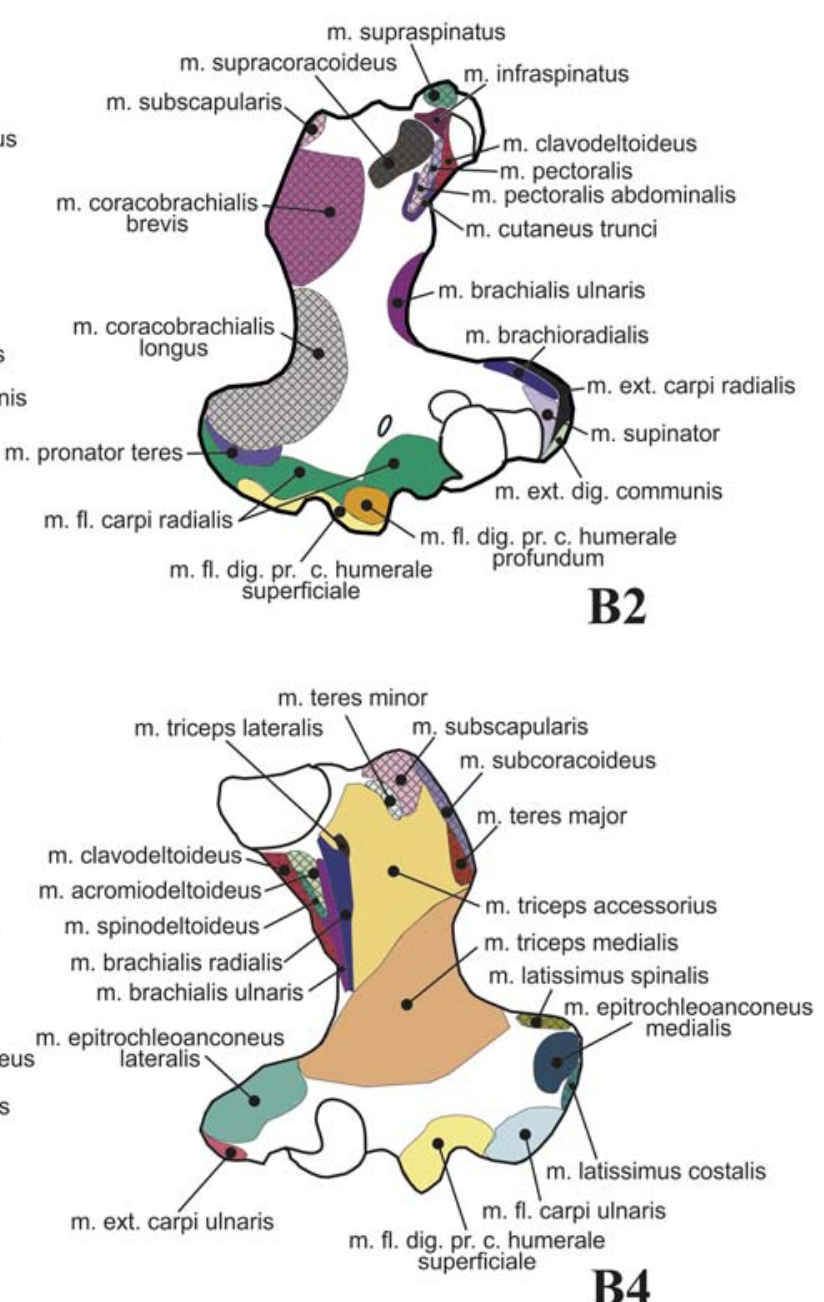

B4

Figure 21 (continued).

follows. The first one, for the $m$. interosseus II medialis, runs from the postaxial side of the base of the metacarpal I to the preaxial side of the tendon II of the m. extensor digitorum communis. The next two ligaments, for the mm. interosseus II lateralis and III medialis, bifurcate to the extensor tendons II and III from the postaxial side of the base of the metacarpal II. The next two ligaments, for the mm. interosseus III lateralis and $I V$ medialis, bifurcate to the extensor tendons III and IV from the postaxial side of the base of the metacarpal III. The last ligament, for the $m$. interosseus IV lateralis, runs from the postaxial side of the base of the metacarpal IV to the postaxial side of the extensor tendon IV.

Remarks. The $m$. interosseus $V$ medialis of Ornithorhynchus looks much different from the other $\mathrm{mm}$. interossei due to its longer muscular belly and unreasonably axial origin, which this muscle shares with the more preaxial $\mathrm{mm}$. interossei $I V$ rather than with its twin, the $m$. interosseus Vlateralis. That is why, the $m$. interosseus $V$ medialis of Ornithorhynchus may be regarded as the only representative of the $\mathrm{mm}$. contra- hentes, usually considered to be lacking in monotremes (Howell, 1936; Jouffroy et al., 1971).

Musculi intermetacarpales (= interossei dorsales) (Fig. 14C) are absent in Tachyglossidae but, contrary to Kajava (1911) and Howell (1936), are present in Ornithorhynchus. Their number is four: two of them are located in the space between the metacarpals II and III, and the other two between the metacarpals III and IV.

Origin (Fig. 23C). The first muscle of this series originates fleshy from the postaxial side of the metacarpal II, the second - from the preaxial side of the metacarpal III, the third - from the postaxial side of the metacarpal III, and the fourth - from the preaxial side of the metacarpal IV.

Insertion (Fig. 23C). All muscles are unipennate. The fibres insert on the tendon, which is parallel to the metacarpal of origin and descends along the same digit to terminate at the end of the phalanx 1 , on the same side as the origin. So, the digit II bears postaxial muscle of this series, the digit IV bears preaxial one, and the digit III bears both of them. 


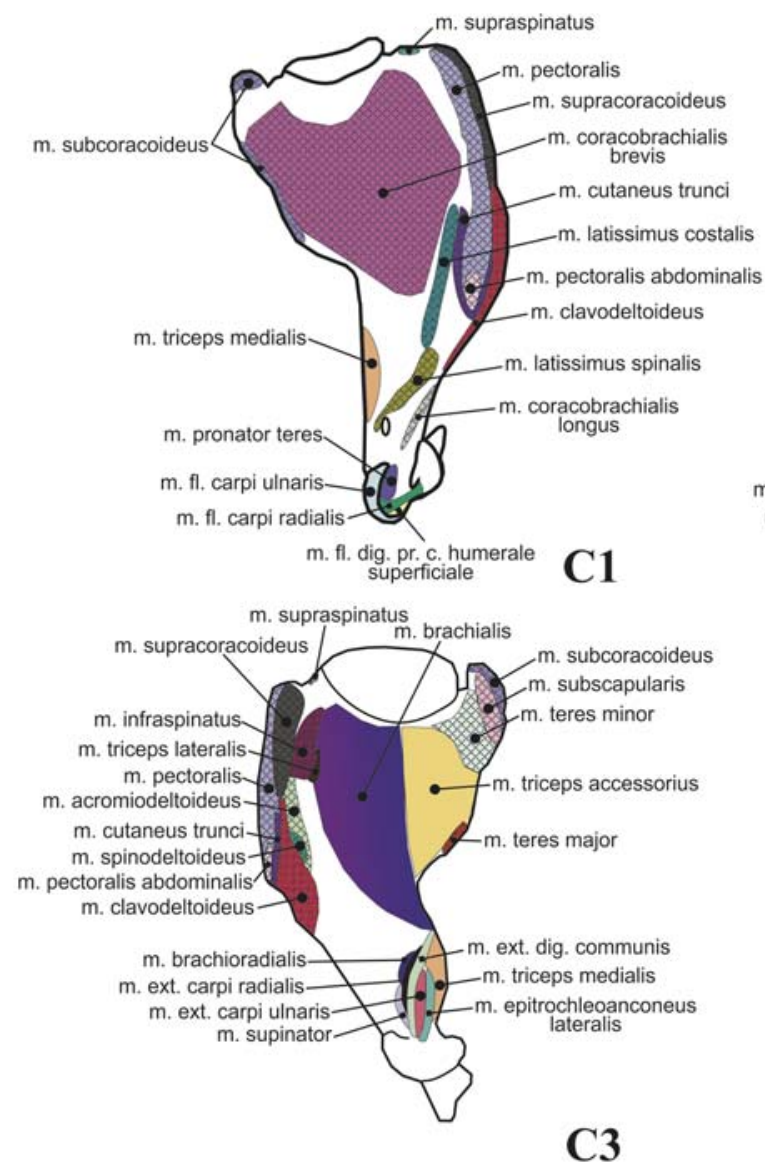

Figure 21 (continued).

\section{Conclusions}

Primitive features of monotremes as a whole relative to therians include: the presence of the $m$. levator scapulae intermedius and $m$. serratus ventralis cervicis accessorius, the underdeveloped $m$. serratus ventralis thoracis vs. cervicis, the development of muscles associated with the coracoid plate (i.e., the $m$. sternocoracoideus, m. costocoracoideus, $m$. subcoracoideus, $m$. supracoracoideus), the single undivided $m$. pectoralis superficialis et profundus, the division of the m. brachialis into ulnar and radial heads, the symmetry of insertion of the $m$. extensor carpi radialis relative to the mid-axis of the metacarpus (i.e., the metacarpal III), the integrity of the $m$. extensor pollicis et indicis, the absence of the $m$. palmaris longus (represented in Zaglossus by a minor fibre bundle of the m. cutaneus trunci), and the location of the bellies of the $m$. flexor digitorum superficialis within the manus.

Advanced features of monotremes as a whole relative to therians include: the extremely distal insertion of the $m$. latissimus dorsi (due to the swollen rib cage) and the presence of the separate $m$. latissimus costalis, the postaxial insertion of the $m$. teres minor, the division of the $m$. triceps longus into two heads, and extremely

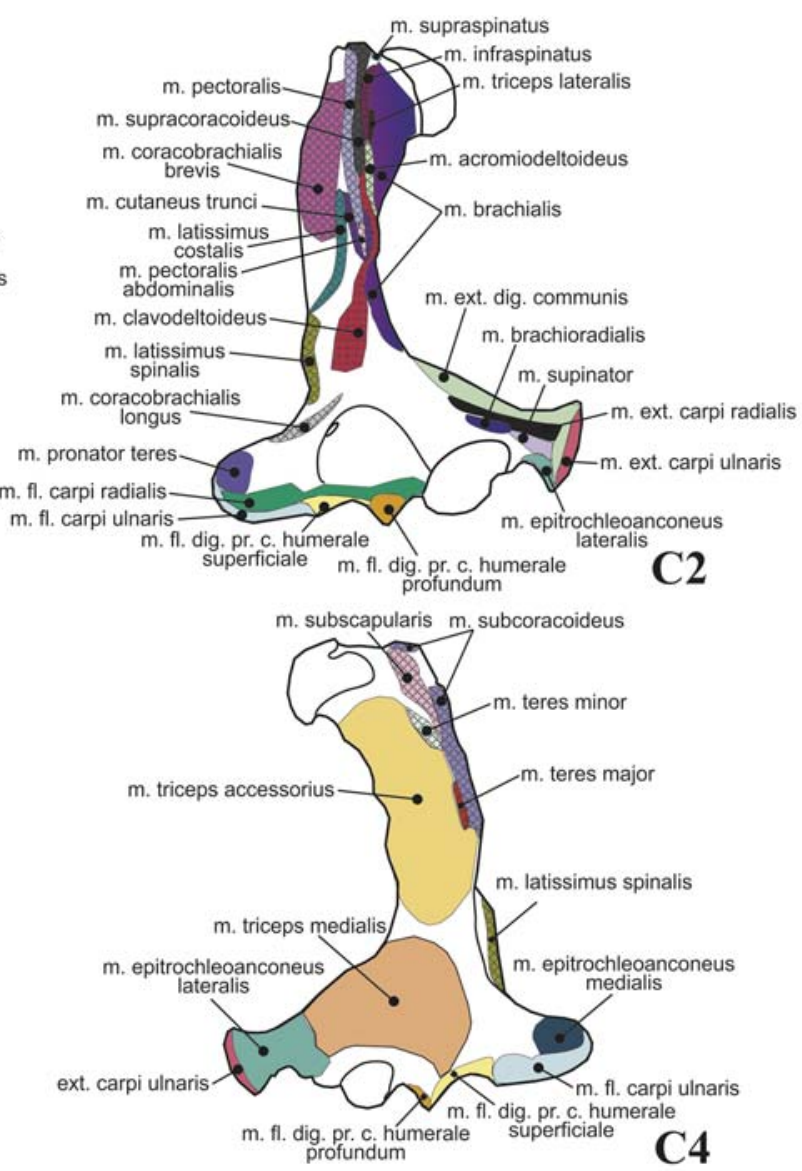

distal insertion of the m. extensor carpi ulnaris, which almost reaches the end of the finger $\mathrm{V}$.

Primitive features of Tachyglossidae relative to $\mathrm{Or}$ nithorhynchus include: the origin of the $m$. levator scapulae beyond the atlas, the less pronounced division of the $m$. serratus ventralis cervicis proprius and accessorius, the unity of the $m$. tensor fasciae antebrachii with the $m$. latissimus costalis, and the fact that the inner surface of the scapula is occupied by the well developed $m$. supraspinatus rather than by the $m$. subscapularis.

Primitive features of Ornithorhynchus relative to Tachyglossidae include: the retention of greater similarity of the $m$. flexor digitorum profundus caput humerale profundum with the amphibian $m$. ulnocarpalis, including the retention of the ligamentum flexorium commune transversum, the full set of the mm. interossei, one of which may be even the $m$. contrahens $V$, and the presence of the $\mathrm{mm}$. intermetacarpales.

In a summary, Tachyglossidae are more primitive in the proximal part of the forelimb (above the elbow), and Ornithorhynchus is more primitive in its distal part. However, Zaglossus shows greater individual variation below the elbow - in the set of the heads of the short flexors (the $\mathrm{mm}$. flexores breves superficiales, $\mathrm{mm}$. lumbricales, $\mathrm{mm}$. interossei) and in the terminal branch- 


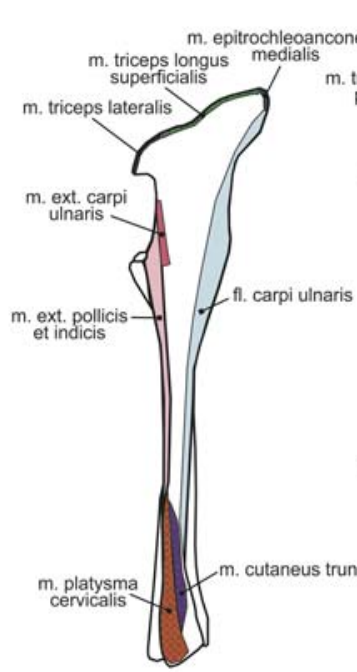

A1
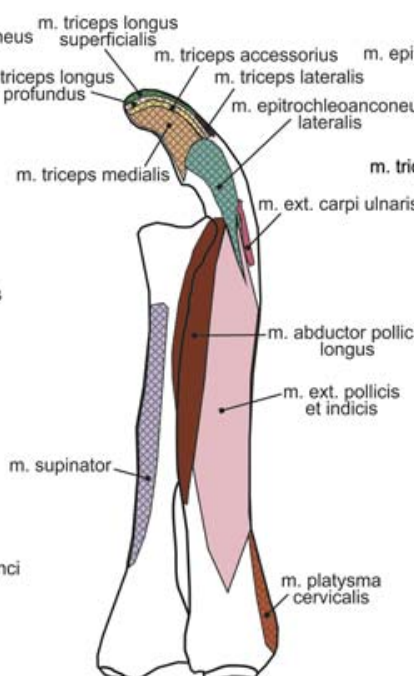

A2

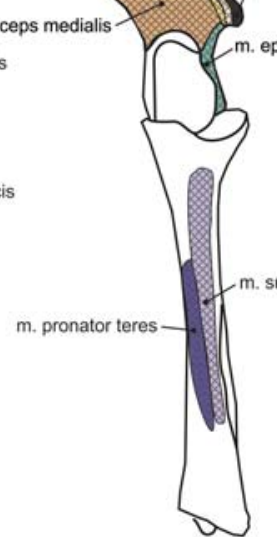

A3

A4

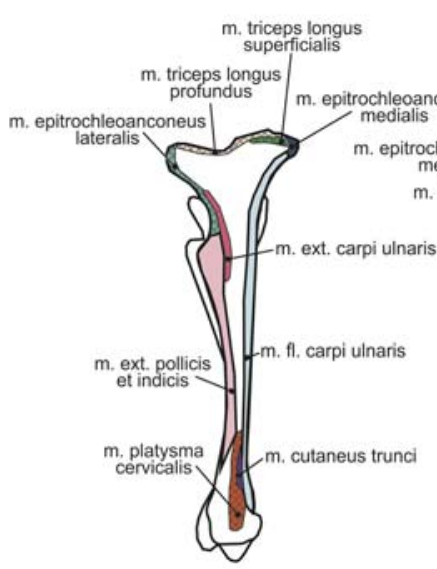

B1

\section{m. triceps accessorius
m. triceps lonnus} m. triceps longus
superficialis epitrochleoanconeus
lateralis m. brachialis ulnaris m. fl. dig. pr. c. ulnare t $m$. brachialis radialis m. fi. carpi ulnaris
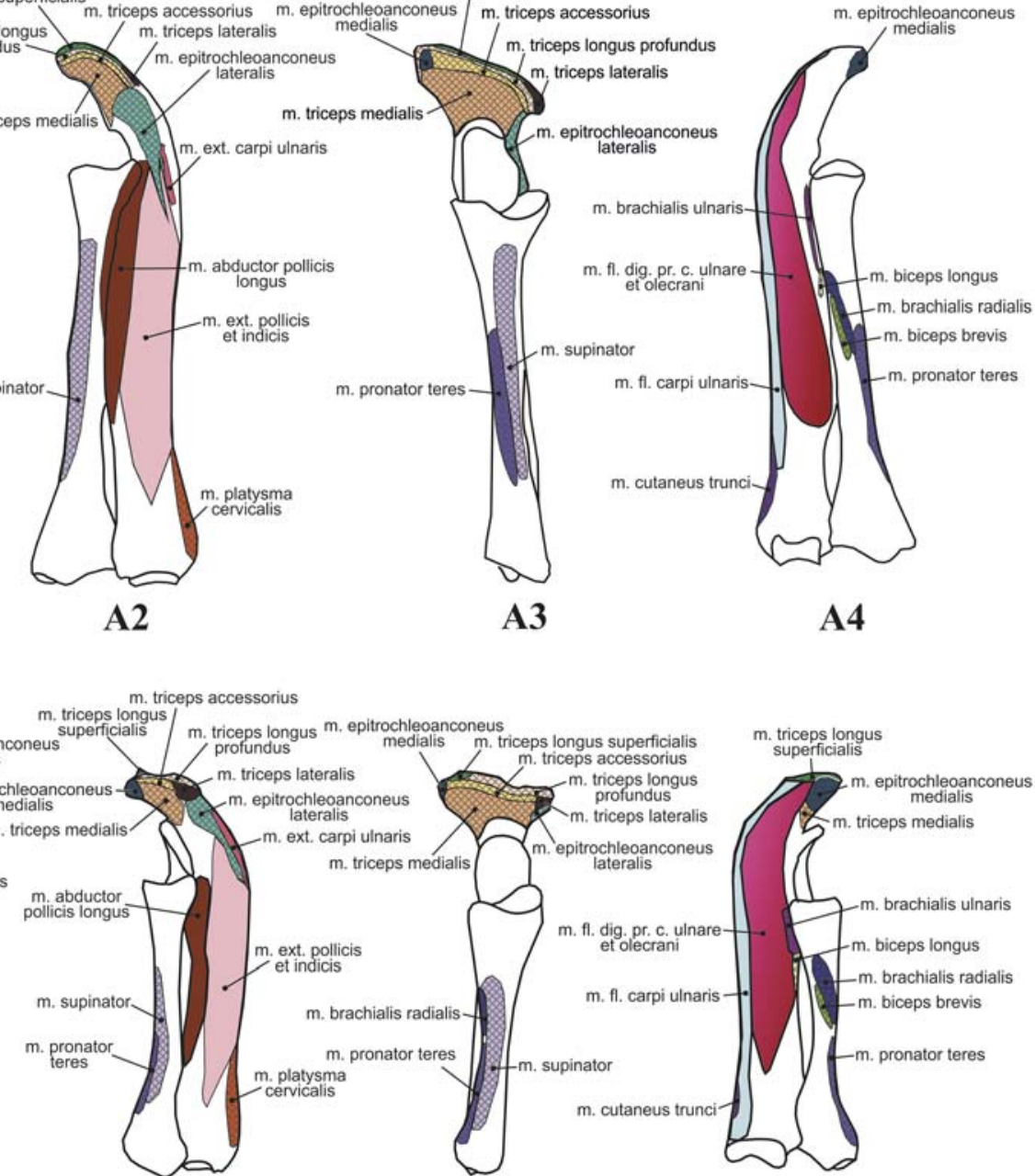

B2

B3

B4

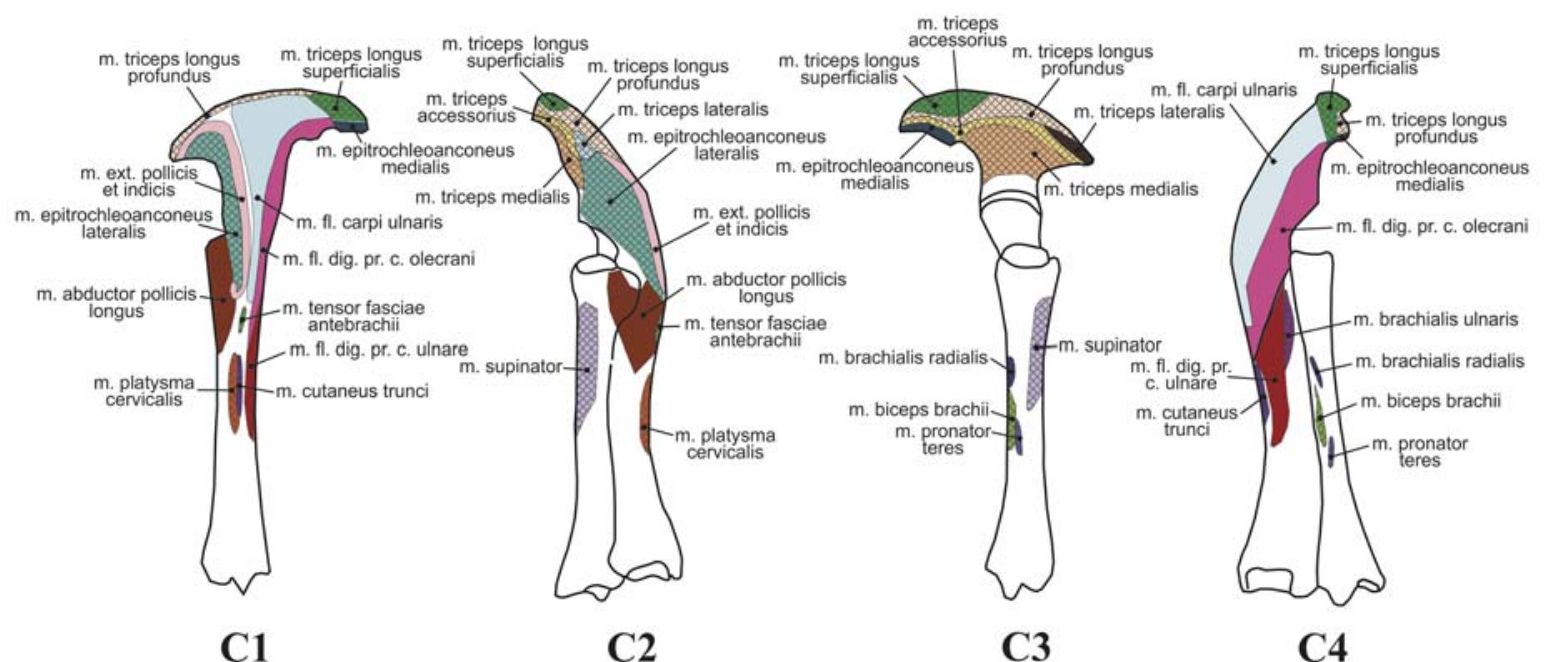

Figure 22. Muscular attachments on the left antebrachium. 1 - postaxial side, 2 - anterior side, 3 - preaxial side, 4 posterior side. 


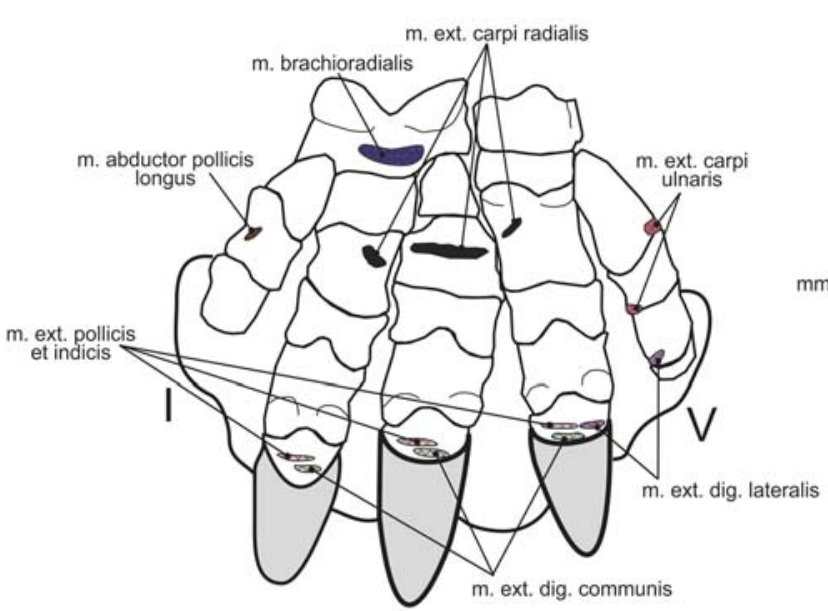

A1

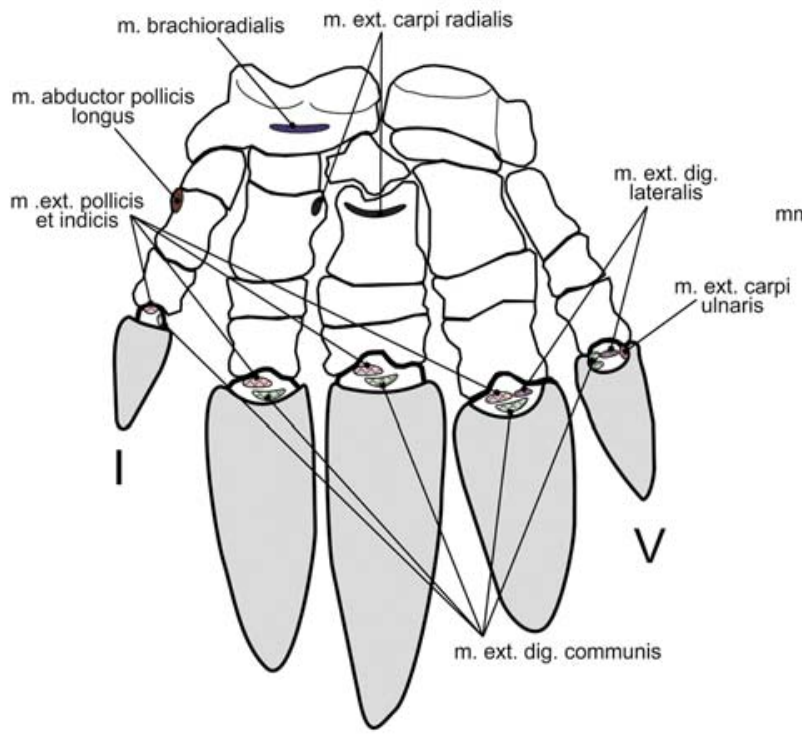

B1

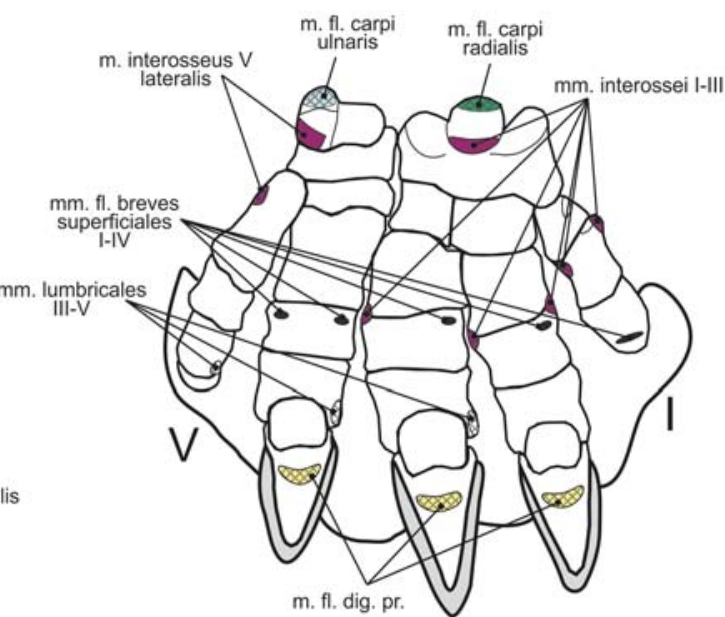

A2

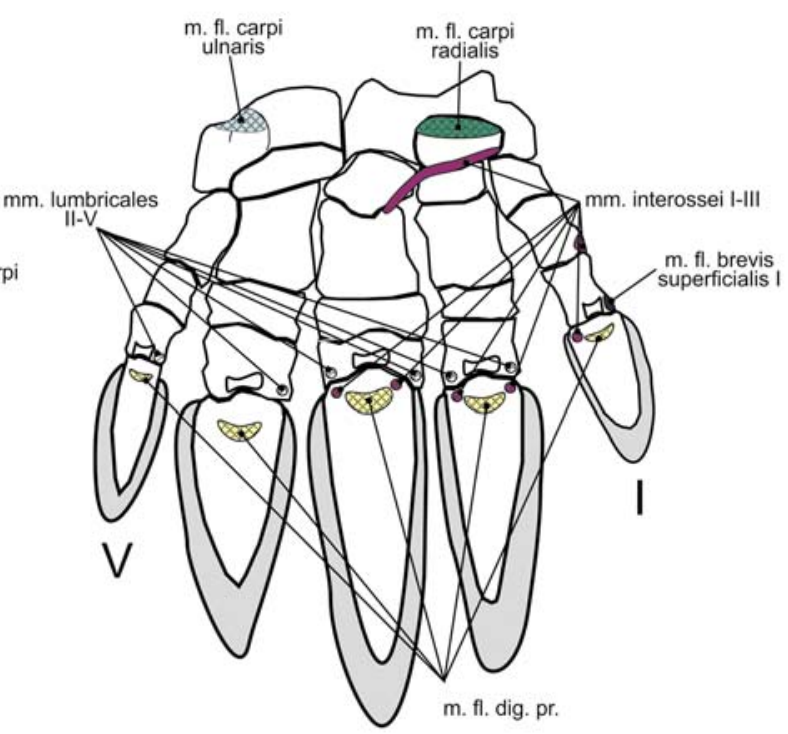

B2

Figure 23. Muscular attachments on the left manus 1 - dorsal view, 2 - palmar view. For Zaglossus, only one individual variation of flexor attachments is shown; it corresponds to Figure 18A.

ing of the $m$. flexor digitorum profundus; this is due to variable degree of reduction of the marginal fingers in this genus. Tachyglossus shows the peculiar bifurcation of the mm. lumbricales.

In some features, monotremes are the most primitive among all living amniotes. Indeed, their $m$. flexor digitorum profundus caput humerale profundum shows traces of the amphibian $m$. ulnocarpalis, and the homonomic subdivision of the $m$. serratus ventralis cervicis into the main and accessory portions overwhelms by its primitivity even amphibians, at least the recent ones.

On the whole, we cannot agree with the opinion of
Howell (1936) that: "the myology of Ornithorhynchus is so specialized that it is misleading to use it in deducing the primitive mammalian arrangement". Among living tetrapods, one cannot find a better model for reconstruction of the locomotorium of the mammal-like reptiles than monotremes, except for their peculiar, anuran-like, ball-and socket elbow joint. In its turn, among monotremes, Zaglossus is definitely the best as such model, except for reduction of marginal fingers. In this respect, the eastern Zaglossus bartoni, which retains all five claws (Flannery \& Groves, 1998), may be even better than our Zaglossus bruijnii. 


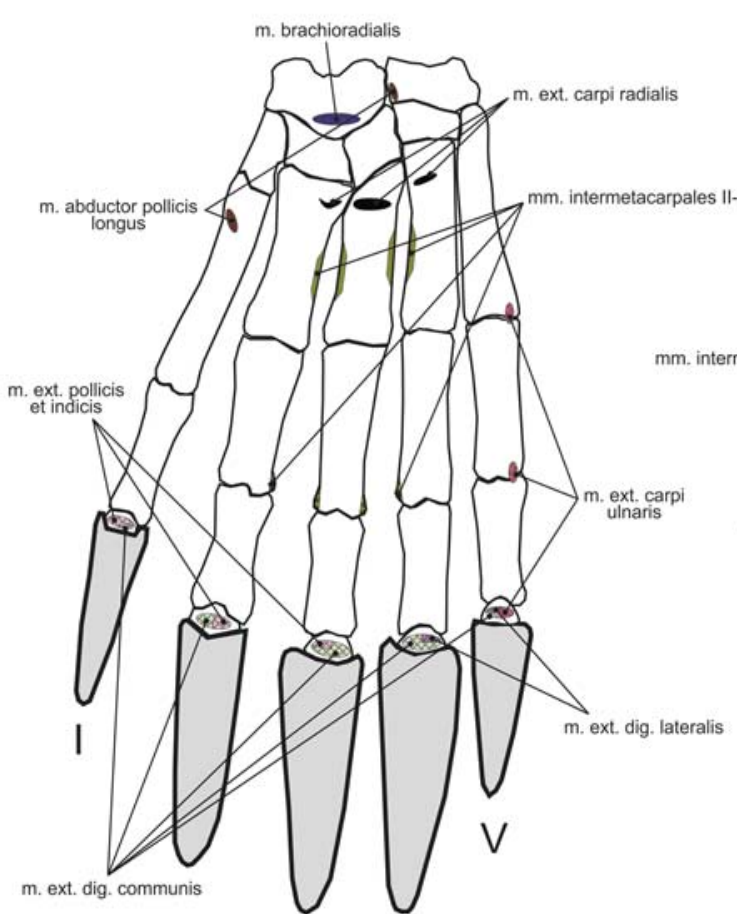

C1

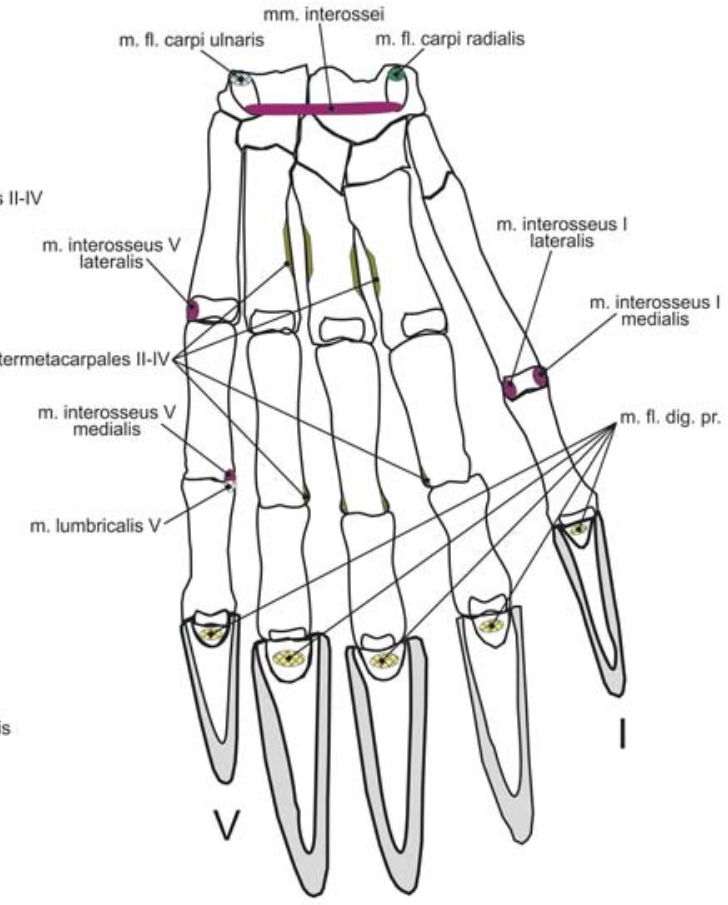

C2

Figure 23 (continued).

ACKNOWLEDGEMENTS. We are obliged to Joan M. Dixon for the specimens of Tachyglossus and Ornithorhynchus, to Fedor V. Terlov and Alexander B. Savinetsky for assistance in preparation of some illustrations, and to Dmitry I. Pashchenko for checking the Latin terminology. This work was partly supported by the Russian Foundation for Basic Research [grants \#\# 14-04-01132-a, 15-04-0504915-a].

\section{References}

Allen G.M. 1912. Zaglossus // Memoirs of the Museum of Comparative Zoology, Harvard College. Vol.40. P.253307.

Diogo R. \& Abdala V. 2010. Muscles of Vertebrates. Comparative Anatomy, Evolution, Homologies and Development. Enfield, New Hapshire: Science publishers. 470 pp.

Ercoli M.D., Álvarez A., Stefanini M.I., Busker F. \& Morales M.M. 2015. Muscular anatomy of the forelimbs of the lesser grison (Galictis cuja), and a functional and phylogenetic overview of Mustelidae and other Caniformia // Journal of Mammalian Evolution. Vol.22. No.1. P.57-91.

Fewkes J.W. 1877. Contributions to the myology of Tachyglossus hystrix, Echidna hystrix (Auct.) // Bulletin of the Essex Institute. Vol.9. P.111-137.

Flannery T.F. \& Groves C.P. 1998. A revision of the genus Zaglossus (Monotremata, Tachyglossidae), with description of new species and subspecies // Mammalia. T.62. No.3. P.367-396.
Haines R.W. 1939. A revision of the extensor muscles of the forearm in tetrapods // Journal of Anatatomy. Vol.73. Pt.2. P.211-233.

Haines R.W. 1950. The flexor muscles of the forearm and hand in lizards and mammals // Journal of Anatatomy. Vol.84. Pt.1. P.13-29.

Holmgren N. 1952. An embryological analysis of the mammalian carpus and its bearing upon the question of the origin of the tetrapod limb // Acta Zoologica. Vol.33. P.1-115.

Howell A.B. 1936. The musculature of antebrachium and manus in the platypus // American Journal of Anatomy. Vol.59. Pt.3. P.425-432.

Howell A.B. 1937. Morphogenesis of the shoulder architecture. Part V. Monotremata // Quarterly Review of Biology. Vol.12. No.2. P.191-205.

Jouffroy F.K., Lessertisseur J. \& Saban R. 1971. Particularités Musculaires des Monotrèmes // Grassé P.-P. (ed.). Traité de Zoologie. T.16. Fasc.3. Paris: Masson et Cie. P.679-836.

Kajava Y. 1911. Die kurzen Muskeln und die langen Beugemuskeln der Säugetierhand. I. Monotremata und Marsupialia // Anatomische Hefte. Bd.42. P.1-194.

McKay W.J.S. 1894. The morphology of the muscles of the shoulder-girdle in monotremes // Proceedings pof the Linnean Society of New South Wales. Vol.9. P.263-360.

Meckel J.F. 1826. Ornithorhynchi Paradoxi Descriptio Anatomica. Leipzig: Gerard Fleisher. 63 pp.

Miner R.W. 1925. The pectoral limb of Eryops and other primitive tetrapods // Bulletin of the American Museum of Natural History. Vol.51. P.145-312. 
Panyutina A.A., Korzun L.P. \& Kuznetsov A.N. 2015. Flight of Mammals: From Terrestrial Limbs to Wings. Springer. 296 pp.

Ponssa M.L., Goldberg J. \& Abdala V. 2010. Sesamoids in anurans: new data, old issues // Anatomical Record. Vol.293. No.10. P.1646-1668.

Romer A.S. 1922. The locomotor apparatus of certain primitive and mammal-like reptiles // Bulletin of the American Museum of Natural History. Vol.46. P.517-606.

Sánchez-Villagra M. R. \& Maier W. 2002. Ontogenetic data and the evolutionary origin of the mammalian scapula // Naturwissenschaften. Vol.89. No.10. P.459-461.
Shrivastava R.K. 1962. The deltoid musculature of the Monotremata // American Midland Naturalist. Vol.67. No.2. P.434-440.

Straus W.L., Jr. 1942. The homologies of the forearm flexors: urodeles, lizards, mammals // American Journal of Anatomy. Vol.70. No.2. P.281-316.

Walter L.R. 1988. Appendicular musculature in the echidna, Tachyglossus aculeatus (Monotremata, Tachyglossidae) // Australian Journal of Zoology. Vol.36. No.1. P.65-81. Westling C. 1889. Anatomische Untersuchungen über Echidna // Bihang till Kongliga Svenska vetenskaps-akademiens handlingar. Bd.15. P.1-71. 